Conversion and Management

Manuscript Number: ECM-D-17-04321R1

Title: Energy and exergy analyses of a parabolic trough collector operated with nanofluids for medium and high temperature applications

Article Type: Original research paper

Section/Category: 1. Energy Conservation and Efficient Utilization

Keywords: Nanofluid; PTC; heat transfer; energy efficiency; exergy efficiency

Corresponding Author: Dr. Amine ALLOUHI, Ph.D

Corresponding Author's Institution: Université Sidi Mohamed Ben Abdellah

First Author: Amine ALLOUHI, Ph.D

Order of Authors: Amine ALLOUHI, Ph.D; M. Benzakour Amine; R. Saidur; T. Kousksou; A. Jamil

Abstract: Thermal performance of parabolic trough collectors (PTCs) can be improved by suspending nanoparticles into the traditionally used heat transfer fluids. In this work, a one-dimensional mathematical model is proposed to investigate the effect of various nanoprticles suspended in the working fluid for medium and high temperature PTCs. The major finding of this work is that the nanofluid enhance the thermal efficiency of the PTCs slightly. High operating temperatures are more suitable for using nanofluids and generates higher relative gains of energy delivered. It is also found that the exergetic efficiency improvement is more important than energetic efficiency. The peak exergy efficiency is achieved by the $\mathrm{CuO}$ based nanofluid and is about 9.05\%. The maximum daily relative gain of thermal energy delivered is found to be $1.46 \%$ by using 5\% of Al203 in the base fluid. Optimal control of the operating conditions can lead to optimal energetic and exergetic performances of the PTC. 
Amine ALLOUHI

École Supérieure de Technologie de Fès, U.S.M.B.A., Route d'Imouzzer BP 2427, Fez,

Morocco

Dear editor-in-chief,

I am pleased to submit this work to you to be considered for publication in "ENERGY CONVERSION AND MANAGEMENT"

I remain at your disposal to provide any further information.

Thank you

ALLOUHI Amine

allouhiamine@gmail.com 


\section{Highlights:}

- Enhancement of PTC performance by using nanofluids

- High operating temperatures are more suitable for using nanofluids in PTCs

- Exergy efficiency of $\mathrm{CuO}$ based nanofluid and is about $9.05 \%$.

- $1.46 \%$ more thermal energy can be generated by using $5 \%$ of $\mathrm{Al}_{2} \mathrm{O}_{3}$ in the base fluid 


\title{
Energy and exergy analyses of a parabolic trough collector operated with nanofluids for medium and high temperature applications
}
A. Allouhi ${ }^{*}(\mathrm{a}), \mathrm{M}$
M. Benzakour Amine ${ }^{(b)}$,
R. Saidu
T. Kousksou ${ }^{(e)}$, A. Jamil ${ }^{(a)}$

(a) Ecole Supérieure de Technologie de Fès, U.S.M.B.A, Route d'Imouzzer, BP 2427, Fez, Morocco

(b) Faculté des Sciences et Techniques de Fès, U.S.M.B.A, Route d'Imouzzer, BP 2202, $\mathrm{Fez}$, Morocco

(c) Research Centre for Nano-Materials and Energy Technology (RCNMET), School of Science and Technology, Sunway University, No. 5, Jalan Universiti, Bandar Sunway, Petaling Jaya, 47500 Selangor Darul Ehsan, Malaysia

(d) Department of Engineering, Lancaster University, Lancaster, LA1 4YW, UK

(e) Laboratoire des Sciences de l'Ingénieur Appliquées à la Mécanique et au Génie Electrique (SIAME), Université de Pau et des Pays de l'Adour - IFR - A. Jules Ferry, $64000 \mathrm{Pau}$, France

\begin{abstract}
:
Thermal performance of parabolic trough collectors (PTCs) can be improved by suspending nanoparticles into the traditionally used heat transfer fluids. In this work, a one-dimensional mathematical model is proposed to investigate the effect of various nanoprticles suspended in the working fluid for medium and high temperature PTCs. The major finding of this work is that the nanofluid enhances the thermal efficiency of the PTCs slightly. High operating temperatures are more suitable for using nanofluids and generate higher relative gains of energy delivered. It is also found that the exergetic efficiency improvement is more important than energetic efficiency. The peak exergy efficiency is achieved by the $\mathrm{CuO}$ based nanofluid and is about $9.05 \%$. The maximum daily relative gain of thermal energy delivered is found to be $1.46 \%$ by using $5 \%$ of $\mathrm{Al}_{2} \mathrm{O}_{3}$ in the base fluid. Optimal control of the operating conditions can lead to optimal energetic and exergetic performances of the PTC.
\end{abstract}

Keywords: Nanofluid; PTC; heat transfer; energy efficiency; exergy efficiency 


\begin{tabular}{|c|c|c|}
\hline Symbol & Signification & Units \\
\hline $\mathrm{h}$ & Hour angle & degree \\
\hline$\delta$ & Solar declination & degree \\
\hline$\theta$ & Incidence angle & degree \\
\hline $\mathrm{k}_{\theta}$ & Incident angle modifier & dimensionless \\
\hline$\epsilon$ & Emittance & dimensionless \\
\hline $\mathrm{G}_{\mathrm{bt}}$ & Solar beam radiation & $\mathrm{W} / \mathrm{m}^{2}$ \\
\hline $\mathrm{c}$ & Specific heat capacity & $\mathrm{J} / \mathrm{kg} \mathrm{K}$ \\
\hline$h_{f}$ & $\begin{array}{l}\text { Convective heat transfer coefficient between the absorber and } \\
\text { the HTF }\end{array}$ & $\mathrm{W} / \mathrm{m}^{2} \mathrm{~K}$ \\
\hline$h_{w}$ & $\begin{array}{l}\text { Convective heat transfer coefficient between the external } \\
\text { surface of the glass cover and the ambient air }\end{array}$ & $\mathrm{W} / \mathrm{m}^{2} \mathrm{~K}$ \\
\hline$\lambda$ & Thermal conductivity & $\mathrm{W} / \mathrm{m} \mathrm{K}$ \\
\hline $\mathrm{k}_{\mathrm{eff}}$ & $\begin{array}{l}\text { effective conductive coefficient between the glass cover and } \\
\text { absorber }\end{array}$ & $\mathrm{W} / \mathrm{m} \mathrm{K}$ \\
\hline $\mathrm{Nu}$ & Nusselt number & dimensionless \\
\hline $\operatorname{Pr}$ & Prandtl number & dimensionless \\
\hline $\mathrm{Pe}$ & Peclet number & dimensionless \\
\hline $\operatorname{Re}$ & Reynolds number & dimensionless \\
\hline $\mathrm{T}$ & temperature & $\mathrm{K}$ \\
\hline $\mathrm{v}$ & velocity & $\mathrm{m} / \mathrm{s}$ \\
\hline$\gamma$ & Intercept factor & dimensionless \\
\hline$\tau$ & transmittance & dimensionless \\
\hline$\alpha$ & absorbance coefficient & dimensionless \\
\hline $\mathrm{r}_{\mathrm{m}}$ & Reflectance of the mirror & dimensionless \\
\hline$\mu$ & DynamicViscosity & $\mathrm{kg} / \mathrm{m} \mathrm{s}$ \\
\hline$\rho$ & Density & $\mathrm{kg} / \mathrm{m}^{3}$ \\
\hline$\sigma$ & Stefan-Boltzman constant & $\mathrm{W} / \mathrm{m}^{2} \mathrm{~K}^{4}$ \\
\hline$\dot{m}$ & Fluid mass flow & $\mathrm{kg} / \mathrm{s}$ \\
\hline $\mathrm{W}_{\mathrm{a}}$ & Width of the collector & $\mathrm{m}$ \\
\hline $\mathrm{L}$ & Length of the collector & $\mathrm{m}$ \\
\hline $\mathrm{D}$ & Diameter & $\mathrm{m}$ \\
\hline A & Cross sectional area & $\mathrm{m}^{2}$ \\
\hline$\phi$ & fraction of nanoparticles & dimensionless \\
\hline$\eta$ & energetic efficiency & dimensionless \\
\hline$\eta_{\mathrm{ex}}$ & exergetic efficiency & dimensionless \\
\hline$\Delta \mathrm{e}$ & relative energy gain & dimensionless \\
\hline FoM & figure of merit & dimensionless \\
\hline
\end{tabular}




\begin{tabular}{|ll|}
\hline Subscripts & \\
$\mathrm{a}$ & Ambient \\
$\mathrm{ab}$ & Absorber \\
$\mathrm{bf}$ & Base fluid \\
$\mathrm{f}$ & Working fluid \\
$\mathrm{g}$ & Glass cover \\
$\mathrm{i}$ & Inner \\
in & Inlet \\
$\mathrm{nf}$ & Nanofluid \\
$\mathrm{np}$ & Nanoparticle \\
o & Outer \\
out & Outlet \\
$\mathrm{s}$ & Solid nanoparticle \\
Abbreviations & \\
HTF & Heat transfer fluid \\
PTC & Parabolic trough collector \\
\hline
\end{tabular}




\section{Introduction}

Concerns regarding climate change are growing and the world needs to take urgent measures to avoid further warming of the earth [1]. The damaging effects of climate change are accentuated with the use of fossil fuels that are up to now considered as the main energy source for power generation worldwide [2]. As a result, increasing efforts are deployed by the research community to propose efficient and reliable alternatives for power generation mainly based on renewable energy sources [3]. Among these renewable energy resources, it is strongly believed that solar energy has the most influential potential to achieve a sustainable global energy system because of many reasons. It is clean, abundant and becoming more and more cost-effective [4]. Solar energy is one of the sustainable and potential options to fulfill a wide range of the humankind daily needs, including natural lighting [5], space and water heating [6-7], cooling [8], water desalination [9] and power generation [10]. Electrical power can be generated using photovoltaic panels by converting solar energy or solar thermal systems driven by thermodynamic cycles. The main advantages of thermal power generation over the PV one rely on the easiness of storing heat compared to electricity and the capability of thermal systems to reach higher energy productions [11]. The current available technologies used in thermal energy plants include, parabolic trough collectors [12], solar towers [13], linear Fresnel lenses [14] and dish Stirling [15]. The use of parabolic trough collectors has been successfully tested in many power generation stations worldwide due to its technological maturity and its economic competitiveness [16-18].

Recently, research related to PTCs has increased tremendously. Many researches proposed improvements in order to ameliorate the performance of PTCs. Some of them focused on proposing modifications in the absorber geometry and including objects inside the flow. Twisted tape inserts were used by Jaramillo et al. [19]. In the case of a twist ratio close to 1 and for low Reynolds numbers, their applications showed a positive effect on the performance of the collector via an enhancement of the heat transfer. Bortolato et al. [20] have studied experimentally a PTC with flat bar-and-plate absorber including an internal offset strip turbulator in the channel. The new design allowed a better efficiency (up to 64\%) with low pressure drops. Other investigators tried to test innovative working fluids such as supercritical $\mathrm{CO}_{2}$ [21] and nanofluids [22-28]. The literature review of the recently published research works has shown that there are only limited works investigating detailed analysis of PTC using nanofluids. Sokhansefat et al. [22] were the first authors to study the possibility of 
improving heat transfer in PTCs by selecting $\mathrm{Al}_{2} \mathrm{O}_{3}$ /synthetic oil nanofluid as a working fluid. A 3-D numerical model based on Navier-Stokes mass, momentum and energy equations were proposed to characterize a fully developed turbulent mixed convection heat transfer through the receiver tube. Authors reported that increasing the concentration of $\mathrm{Al}_{2} \mathrm{O}_{3}$ nanoparticles up to $5 \%$ may increase the heat transfer coefficient by $14 \%$. Ghesemi and Ranjbar [23] simulated the thermal behavior of a PTC using $\mathrm{CuO}$-water and $\mathrm{Al}_{2} \mathrm{O}_{3}$-water nanofluids. The numerical model is based on the finite volume approach and solved by a CFD commercial code. It is shown that the tested nanofluids gave better performances compared to pure water. For a volume fraction of $3 \%$, they reported an increase in the heat transfer coefficient of about $28 \%$ and $35 \%$ for $\mathrm{CuO}$-water and $\mathrm{Al}_{2} \mathrm{O}_{3}$-water nanofluids, respectively. Mwesigye et al. [24] investigated numerically the thermal and thermodynamic performance of a high concentration ratio PTC employing $\mathrm{Cu}$-Therminol VP-1 nanofluid as the working fluid. The conclusion given by the authors is that the collectors' thermal efficiency increased to $12.5 \%$ when the nanoparticle concentration varied between 0 to $6 \%$. They have also shown that by using the entropy generation method, the nanofluids can enhance thermodynamic efficiency for the certain range of Reynolds numbers. Bellos et al. [25] analyzed theoretically two options for enhancing thermal efficiency of PTCs. The first option consists of considering a dimpled receiver with a sine form. For the second option, they compared three working fluids and nanofluid was one of them. They argued that both approaches can improve the efficiency by around 4\%. An optic-thermal-stress coupling model was suggested by Wang et al. [26] in order to evaluate the influence of using $\mathrm{Al}_{2} \mathrm{O}_{3} /$ synthetic oil nanofluid as a working fluid in PTCs. The authors indicated that nanofluids enhance heat transfer, avoid high temperature gradients and minimize thermal stress receiver's deformation. Simulations were carried out by Coccia et al. [27] to analyze the energy yields of low-enthalpy parabolic trough collectors utilizing six water-based nanofluids. The authors concluded that adding low concentrations of some nanofluids lead only to minor improvements in the PTC efficiencies while high concentrations do not induce an advantage compared to water. This result originates from the fact that the dynamic viscosity increases with the weight concentration. They have therefore recommended that evaluating nanofluids (as working fluids in PTCs) at high temperatures (characterized by lower dynamic viscosities and higher thermal conductivities) could be interesting.

Based on literature survey, it was found that there are only limited investigations studying the thermal behavior of PTCs operating with nanofluids. More works with detailed analysis are 
therefore required for a good understanding of the best conditions of using nanofluids in PTC applications. Moreover, the assessment of their benefits seems to be of a particular interest, especially for medium and high temperature applications as emphasized by [27]. Another key contribution of this paper is the discussion of the effect of nanofluids on the exergetic performance of PTCs. Very limited studies were carried out on this aspect as well. In this sense, the present work presents a thermal analysis and performance assessment of PTC using three types of nanofluids as heat transfer fluids for medium and high temperature applications. The proposed mathematical model is one-dimensional and takes into account real varying external conditions in terms of incident radiation and ambient temperature for the Moroccan city "Ouarzazate". A parametric study was also conducted to show the effect of mass flow rate, inlet temperature and nanoparticle concentration on the output energy. Detailed energetic and exergetic analyses are carried out as well to identify the best conditions of nanofluid utilization in PTCs.

\section{Mathematical formalism}

\subsection{Tested fluids}

The mathematical model attempts to study heat transfer and thermal and exergetic efficiencies of a PTC using nanolfluids as working fluids. As the main focus of this paper is on medium and high-temperature heating applications, Therminol VP-1 was used as the base heat transfer fluid which is suitable for such purposes. Temperature dependent thermal properties are required for a more accurate modeling of the system. Hence, the thermal properties varying with the temperature were extracted from the manufacturer datasheet and were fitted under polynomial or exponential equations to be appropriately used by the developed code [28]. Their expressions, by considering only the liquid phase, are given below:

- Density $\left(\mathrm{kg} / \mathrm{m}^{3}\right)$ :

$$
\rho_{b f}=-2.379 \times 10^{-6} \mathrm{~T}^{3}+0.002737 \mathrm{~T}^{2}-1.871 \mathrm{~T}+1439
$$

- Specific heat capacity (J/kg K)

$$
c_{p_{b f}}=8.877 \times 10^{-6} \mathrm{~T}^{3}-0.01234 \mathrm{~T}^{2}+8.28 \mathrm{~T}-50.85
$$

- Thermal conductivity (W/m K)

$$
\lambda_{b f}=1.062 \times 10^{-11} \mathrm{~T}^{3}-1.937 \times 10^{-7} \mathrm{~T}^{2}+2.035 \times 10^{-5} \mathrm{~T}+0.1464
$$


- Dynamic viscosity (Pa s)

$$
\mu_{b f}=30.24 \exp (-0.03133 \mathrm{~T})+0.008808 \exp (-0.006729 \mathrm{~T})
$$

Integrating nanoparticles in the base fluid will induce an enhancement in its thermal properties. These properties are influenced by the volume fraction of the nanoparticles and their typology. Generally, this volume fraction does not exceed 5\%. The nanofluid thermal properties (i.e. density, specific heat capacity, thermal conductivity and dynamic viscosity) as a function of the volume fraction of nanoparticles $(\phi)$, are derived from the next expressions [29-31]:

$\rho_{n f}=(1-\phi) \rho_{b f}+\phi \rho_{s}$

$c_{p_{n f}} \frac{(1-\phi)\left(\rho c_{p}\right)_{b f}+\phi\left(\rho c_{p}\right)_{s}}{\rho_{n f}}$

$\lambda_{n f}=\lambda_{b f} \frac{\lambda_{s}+2 \lambda_{b f}-2 \phi\left(\lambda_{b f}-\lambda_{s}\right)}{\lambda_{s}+2 \lambda_{b f}+\phi\left(\lambda_{b f}-\lambda_{s}\right)}$

$\mu_{n f}=\mu_{b f}\left(1+2.5 \phi+6.25 \phi^{2}\right)$

In the previous equations, the subscript (nf) denotes for nanofluid, (bf) for the base fluid and (s) for the solid nanoparticles.

The study considers three oxide nanopaticle types: copper oxide $(\mathrm{CuO})$, alumina $\left(\mathrm{Al}_{2} \mathrm{O}_{3}\right)$ and titanium oxide $\left(\mathrm{TiO}_{2}\right)$. The thermal properties of these nanoparticles are given in Table 1 [3233].

\subsection{Climatic conditions}

In this work, it is suggested to study the instantaneous thermal performance of a PTC using nanofluids. A typical sunny day has been selected to run the simulation. Ambient temperature and direct beam radiations were obtained from the METEONORM database for the Moroccan city Ouarzazate. To simplify the study, an open-loop operation mode of the PTC has been considered without any coupling with a hot storage tank. This configuration has been previously proposed by Coccia et al. [27]. In the present work, a horizontal E-W axis with N- 
$\mathrm{S}$ single axis tracking is studied. The sun-tracking mechanism depends on the solar incidence angle, denoted $\theta$. The cosine of $\theta$, for a surface rotated about a horizontal east-west direction with regular adjustment is expressed as follows [34]:

$$
\cos (\theta)=\sqrt{1-\cos ^{2}(\delta) \sin ^{2}(h)}
$$

$\delta$ is the solar declination and $\mathrm{h}$ is the hour angle, all expressed in degrees.

It is interesting to note that the climatic conditions were obtained under an hourly form and were introduced into the developed code using a fifth-order polynomial interpolation.

\subsection{PTC modeling}

\subsubsection{Governing equations}

A PTC comprises a parabolic reflecting mirror that reflects the sun rays onto a receiver tube that is inserted at the focal point of the reflector. The receiver consists of a metallic absorber surrounded by a glass cover. To limit heat losses, the space between the glass cover and absorber is maintained at very low pressures. The PTC is schematically reported in Fig. 1 [35].

A one dimensional mathematical model is introduced to study the transient thermal behavior of the PTC. Therefore, the receiver tube is divided into $\mathrm{N}$ segment and heat propagation occurs according the axial direction. The inputs of the model are the instantaneous ambient temperature, incident beam radiations, mass flow rate, and physical properties of the glass cover, absorber tube and HTF.

The mathematical model is based on an energy balance in each segment of the glass envelope, absorber and the HTF. Consequently, it is imperative to compute the various heat transfer coefficients used by the model. Some simplifying hypotheses have been made:

- Incompressible HTF and unidirectional flow

- Fluid flow is uniformly distributed for each receiver segment

- Solar radiation is time dependent and is uniform around the whole receiver tube

- Conduction losses at the ends of receiver tube are neglected.

- Thermal properties of the base fluid vary with the temperature, whereas those of nanoparticles are constant.

- Thermal diffusion term in the glass cover, absorber tube and fluid are negligible 
$\dot{q}_{\text {in }}=\dot{q}_{\text {in-rad }}+\dot{q}_{\text {in-conv }}$

210 The radiation heat transfer mode between the receiver pipe/absorber and glass envelope can

The three coupled partial differential equations referring to the energy balances for the glass cover, absorber tube and working fluid can be expressed as follows:

- Glass cover:

The glass cover receives solar radiation along its outer surface, exchanges heat with both the absorber tube and the ambient. Energy balance for the glass cover is given as:

$$
A_{g} \rho_{g} c_{g} \frac{\partial T_{g}}{\partial t}=\dot{q}_{s-g}(t)+\dot{q}_{\text {in }}(x, t)-\dot{q}_{\text {out }}(x, t)
$$

The solar radiation received by the glass cover $\dot{q}_{s-g}(t)$ can be considered as a heat flux. This can be justified by the fact that the glass cover is significantly thin and possesses a very low absorptance coefficient of the order of 0.02. It can be expressed as:

$\dot{q}_{s-g}(t)=\gamma \alpha_{g} r_{m} W_{a} G_{b t}(t) k_{\theta}(t)$

This term depends on the available instantaneous beam solar radiation $\left(G_{b t}\right)$, collector width $\left(\mathrm{W}_{\mathrm{a}}\right)$ and other optical properties including intercept factor $(\gamma)$, absorbance of glass cover $\left(\alpha_{\mathrm{g}}\right)$, specular reflectance of the mirror $\left(\mathrm{r}_{\mathrm{m}}\right)$ and the incident angle modifier $\left(\mathrm{k}_{\theta}\right)$. The incident angle modifier is given as a fourth-order polynomial form of the incident angle [36]:

$k_{\theta}=1-2.2307 \times 10^{-4} \theta-1.1 \times 10^{-4} \theta^{2}+3.18596 \times 10^{-6} \theta^{3}-4.85509 \times 10^{-8} \theta^{4}$

All the parameters of Eq. (11) together with other geometrical properties of the PTC are specified in Table 2 [37].

Internal heat transfer between the absorber and the glass envelope heat transfer occur by convection and radiation, thus: be written as: 
$212 \dot{q}_{i n-r a d}=\frac{\pi D_{o-g}\left(T_{a b}^{4}-T_{g}^{4}\right)}{\frac{1}{\epsilon_{a b}}+\frac{1-\epsilon_{g}}{\epsilon_{g}} \frac{D_{a b-o}}{D_{g-i}}}$

213 Considering that the convection heat transfer mechanism between the receiver pipe and glass

214 envelope occurs by natural convection due to the presence of a pressure $>0.013 \mathrm{~Pa}$, one can

215 use the Raithby and Holland's formula to characterize the convection heat transfer between

216 the absorber tube and glass cover wall [38]

217

$$
\dot{q}_{\text {in-conv }}=\frac{2 \pi k_{e f f}\left(T_{a b}-T_{g}\right)}{\ln \left(\frac{D_{g-i}}{D_{a b-o}}\right)}
$$

Heat exchange between the glass cover and the atmosphere takes place by convection and radiation. Due to the presence of wind, the Newton's law of cooling can be employed to determine the convective heat loss as [34]:

$$
\dot{q}_{\text {out-conv }}=\pi D_{g-o} h_{w}\left(T_{g}-T_{a}\right)
$$

222 with:

223

$$
h_{w}=\frac{N u_{a i r} k_{a i r}}{D_{g-o}}
$$

224 and

225

$$
N u_{\text {air }}=\left\{\begin{array}{l}
0.4+0.54 \operatorname{Re}_{\text {air }}{ }^{0.52} \text { if } 0.1<\operatorname{Re}_{\text {air }}<1000 \\
0.3 \operatorname{Re}_{\text {air }}{ }^{0.6} \text { if } 1000<\operatorname{Re}_{\text {air }}<50000
\end{array}\right.
$$

226 Taking the assumption that the cover is a small convex gray object in a large black body

227 cavity, the sky, one can estimate the radiation heat exchange by:

$$
\dot{q}_{\text {out-rad }}=\pi D_{g-o} \in \in_{g} \sigma\left(T_{g}^{4}-T_{s k y}^{4}\right)
$$

229 In the previous equations $\mathrm{T}_{\mathrm{g}}, \mathrm{T}_{\mathrm{a}}$ and $\mathrm{T}_{\text {sky }}$ correspond to the outer glass cover temperature, ambient temperature, respectively. $\mathrm{T}_{\text {sky }}$ is the sky temperature taken as $T_{s k y}=0.0552 T_{a}^{1.5}$ 
$231 \sigma$ is the Stefan-Boltzman constant $\left(\sigma=5.67 \times 10^{-8} \mathrm{~W} / \mathrm{m}^{2} \mathrm{~K}^{4}\right)$ while $\epsilon_{\mathrm{g}}$ and $\epsilon_{\mathrm{ab}}$ are the emittance

241 The term $\dot{q}_{s-a b}(t)$ refers to the solar energy absorbed by the PTC receiver. It can be put under 242 the following form:

or:

$$
\dot{q}_{s-a b}(t)=\dot{q}_{s-g}(t) \frac{\left(\tau_{g} \alpha_{a b}\right)}{\alpha_{g}}
$$

with $\alpha_{\mathrm{ab}}$ and $\tau_{\mathrm{g}}$ are respectively the absorbance coefficient of the PTC absorber and the glass cover transmittance.

The remaining term in Eq. (20) denotes for the useful heat transmitted to the HTF. This term is the most important parameter when comparing various heat transfer fluids. It can be expressed as:

$\dot{q}_{u}(x, t)=\pi D_{a b-i} h_{f}\left(T_{a b}-T_{f}\right)$

$D_{a b-i}$ is the inner diameter of the absorber and $T_{f}$ is the HTF temperature. $h_{f}$ is the convection heat transfer coefficient between the absorber and the HTF and is strongly dependent on the 
254

255

256

257

258

259

260

261

262

263

264

265

266

267

268

269

270

271

272

273

274

275

276

277

thermal properties of the working fluid. This coefficient is determined based on the Nusselt number value. Here, two correlations are used referring to the case of the base fluid and to the case of nanofluids. The first correlation, depending on Reynolds and Prandtl numbers, called the Dittus-boelter correlation estimates the Nusselt number as follows [39]:

$N u_{b f}=0.023 \operatorname{Re}_{b f}{ }^{0.8} \operatorname{Pr}_{b f}{ }^{0.4}$

In the case of nanofluid, Xuan et al. [40] proposed the following formulation to estimate the Nusselt number:

$N u_{n f}=0.0059\left(1.0+7.628 \phi^{0.6886} P e_{n p}{ }^{0.001}\right) \operatorname{Re}_{n f}{ }^{0.9238} \operatorname{Pr}_{n f}{ }^{0.4}$

where $\mathrm{Pe}_{\mathrm{np}}$ is the Peclet number describing the effect of thermal dispersion because of microconvective and microdiffusion of the suspended nanoparticles. It is given as:

$P e_{n p}=\frac{v_{n f} \times d_{n p}}{\alpha_{n f}}$

with $\mathrm{v}_{\mathrm{nf}}$ is the nanofluid velocity, $\mathrm{d}_{\mathrm{np}}$ is the nanoparticle diameter and $\alpha_{\mathrm{nf}}$ is the thermal diffusivity of nanofluid. Reynolds and Prandtl numbers are evaluated by considering the temperature-dependent thermal properties of each nanofluid type.

It is also interesting to highlight that the two previous correlations are recommended in the case of turbulent flows. In this sense, simulation tests were carried out to determine the mass flow range with respect to this condition.

- Working fluid

The working fluid flows inside the absorber at a flow rate $\dot{m}$ and absorbs heat by convection from the inner absorber tube. The energy balance of the HTF can take the following form:

$A_{f} \rho_{f} c_{f} \frac{\partial T_{f}}{\partial t}+\dot{m} c_{f} \frac{\partial T_{f}}{\partial x}-k_{f} A_{f} \frac{\partial^{2} T_{f}}{\partial x^{2}}=\dot{q}_{u}(x, t)$

In all the governing equations $\mathrm{A}, \rho$ and $\mathrm{c}$ denotes for the cross-sectional area $\left(\mathrm{m}^{2}\right)$, density $\left(\mathrm{kg} / \mathrm{m}^{3}\right)$ and specific heat capacity $(\mathrm{J} / \mathrm{kg} \mathrm{K})$. Also, it is noteworthy to mention that all the equations are referred to the length unit of the collector. 
The initial conditions of the energy balance equations were introduced by considering that at 280 time $\mathrm{t}=0$, the glass cover, absorber tube and HTF are all in thermal equilibrium with the atmosphere. Moreover, the boundary conditions were implemented considering that at $\mathrm{x}=0$, the temperatures are constant and refer to the inlet fluid temperature.

\subsection{Performance indices}

The present work suggests assessing the performance of the solar PTC by comparing the outlet temperature of the working fluid (that can be base fluid or one of tested nanofluids), the energetic efficiency the PTC, its exergetic efficiency and the relative benefit of the useful energy delivered for the various working fluids.

The impact on these indices is the result of the improvement of the heat coefficient transfer $h_{\mathrm{f}}$. The Figure of Merit (FoM) expressing the ratio of the heat transfer coefficient (nanofluid cases and base fluid case) is a useful criterion to judge the benefit of nanofluids versus the base fluid. It is given as [41]:

291

$$
F o M=\frac{\left.h_{6}\right|_{2, f}}{\left.h_{f}\right|_{b f}}
$$

The outlet temperature of the HTF is determined by solving the previous set of equations and corresponds to:

$T_{\text {out }}=T_{f}(x=L)$

The instantaneous energetic efficiency refers to the ratio between the useful thermal energy 298 gained by the working fluid to the available solar beam energy falling onto the PTC reflector. It is expressed as:

$299 \quad \eta=\frac{\dot{Q}_{u}}{A_{a} G_{b t}}=\frac{\dot{m} \int_{T_{i n}}^{T_{\text {out }}} c_{f}(T) d T}{W_{a} L G_{b t}}$ 
The exergetic efficiency can be defined as the ratio of gain exergy $\left(\mathrm{E}_{\mathrm{u}}\right)$ to available solar radiation exergy $\left(\mathrm{E}_{\mathrm{s}}\right)$ and can be expressed as [42]:

302

$$
\eta_{\text {ex }}=\frac{E_{u}}{E_{s}}=\frac{\dot{m} \int_{T_{\text {in }}}^{T_{\text {out }}} c_{f}(T) d T-T_{a} \int_{T_{\text {in }}}^{T_{\text {out }}} \frac{c_{f}(T)}{T} d T}{W_{a} L G_{b t}\left[1-\frac{4}{3}\left(\frac{T_{a}}{T_{\text {sun }}}\right)+\frac{1}{3}\left(\frac{T_{a}}{T_{\text {sun }}}\right)^{4}\right]}
$$

303 304

In Eq. (31), $\mathrm{T}_{\text {sun }}$ is the sun's apparent temperature taken to be $6000 \mathrm{~K}$ as mentioned by Petela [43].

The last performance indicator is the relative energy gain resulting from the difference between the energy delivered by the PTC when the nanofluids are used compared to the base fluid. It is given as

$$
\Delta e=\frac{Q_{u-n f}-Q_{u-b f}}{Q_{u-b f}} \times 100
$$

The flow diagram, showing the inputs, the outputs and the calculations operated by the model is presented in Fig. 2.

Proving the validity of the proposed mathematical model is essential before further exploitation of its results. Therefore, a validation was performed based on a comparison between our model and experimental tests of Sandia National Laboratory (SNL) [44]. The SNL has experimentally tested a small module of LS-2 collector at the AZTRAK rotating platform to analyze the effect of various conditions on the PTC performance which can help in minimizing operation and maintenance costs of CSP plants. The code of the present model has been run in similar conditions as in [44] considering the same working fluid (Syltherm 800 oil) and the same geometrical properties of the PTC. Three test conditions were considered for the validation that is based on the outlet temperature and the thermal efficiency. The results are given in Table 3. It is clear that the results of the model in terms of outlet temperature and thermal efficiency are in very good agreement with the measured data 
(uncertainty $<0.83{ }^{\circ} \mathrm{C}$ for the temperature and $<2.9 \%$ for the efficiency). This proves that the developed mathematical model is valid.

\section{Results and discussion}

Several MATLAB subroutines were built to compute various inputs for the main program. The main program includes the discretization of the differential equations and resolution of the obtained algebraic equations. At each time iteration, the non-linear aspect of the problem is handled by considering the temperature-dependent thermal properties at the previous time step. When the temperature of the glass cover, absorber and HTF are known, the program computes the performance indices on a time-evolution basis.

Climatic input data were load from MS Excel data after a pre-processing of the cosine of incident angle accounting for the sun-tracking strategy (i.e. N-S tracking). As stated before, a typical sunny day in the region of Ouarzazate (Morocco) is considered. The climatic data are depicted in Fig. 3. A maximum ambient temperature of $308 \mathrm{~K}$ is recorded at $15 \mathrm{~h} 00$ am while the minimum one is recorded at the sunrise (291 K). Fig. 3 also shows the hourly variation of the incident beam radiation between the sunrise and the sunset. The peak solar radiation is observed at midday and is about $1000 \mathrm{~W} / \mathrm{m}^{2}$. Other subroutines were developed in order to compute the term sources of the governing equations. The various properties of the tested fluids with respect to the temperature are used at each time step for a more accurate resolution. The generated data are used by the main program and serve in determining the heat transfer coefficient and other involved parameters figuring in the governing equations. Fig. 4 plots thermal properties of the base fluid together with the tested nanofluids for temperatures ranging from $300 \mathrm{~K}$ to $650 \mathrm{~K}$. It is clear that nanofluids possess higher densities than the base fluid (see Fig. 4 (a)). All fluids have a descending behavior of density with increasing temperatures. Increasing the concentration of nanoparticles induces further increase in the density. Also, it is clear that $\mathrm{Cu}-\mathrm{O}$ nanoparticles have a more pronounced effect on the increase of the density if compared to other types. Obviously, the presence of nanofluids leads to an enhancement of the thermal conductivity of HTF, as indicated in Fig. 4 (b). It is shown that $\mathrm{TiO}_{2}$ based nanofluid has a slightly lower thermal conductivity compared to the other nanofluids that have approximately the same values. This is surely because $\mathrm{TiO}_{2}$ nanoparticles have lower thermal conductivity (see Table 1). Moreover, by increasing the concentration of nanoparticles, thermal conductivities increase as well. By increasing the temperature, one can see that the relative gain in terms of the enhancement of the thermal 
conductivity is reduced independently of the nature of nanoparticles. The specific heat capacity, as indicated in Fig. 4 (c), gets decreased by using nanofluids. The most influential effect is shown for the case of $\mathrm{CuO}$ based nanofluid. The two other nanofluids have approximately at low concentration of nanoparticles, but as the concentration of nanoparticles increases, the difference between their specific heat capacities becomes greater.

Fig. 4 (d) shows the variation of dynamic viscosity versus the temperature. The main observation is that, at higher temperatures, adding nanoparticles to the base fluid, have a negligible effect on the viscosity. Also, as the nanoparticle concentration increases, the working fluid becomes more viscous. Such tendency is clearer at low temperatures. The changes on the thermal properties of the working fluids will certainly affect its thermal performance.

Based on these thermal properties, it was possible to generate plots of the convective heat transfer coefficient. Besides, the two correlations of the Nusselt number (Eq. (24) and Eq. (25)) referring to the base fluid case and the nanofluid case were used in the computational procedure. Fig. 5 shows the trend of this coefficient for various operating conditions, considering the case of the base fluid. It is seen that the heat convection coefficient increases with increasing temperatures (from $120 \mathrm{~W} / \mathrm{m}^{2} \mathrm{~K}$ at $300 \mathrm{~K}$ to $420 \mathrm{~W} / \mathrm{m}^{2} \mathrm{~K}$ at $650 \mathrm{~K}$ ). The curve slope is a little more important for temperatures $<400 \mathrm{~K}$.

For the sake of comparison, a 3-D representation showing the variation of the convective heat transfer coefficient in the case of the $\mathrm{CuO}$ based nanofluid is illustrated in Fig. 6. It can be clearly seen that the presence of $\mathrm{CuO}$ nanoparticles considerably enhances the convective heat transfer coefficient. This enhancement is of the order of $32 \%-83 \%$ at a maximum operating temperature of $650 \mathrm{~K}$, when compared to the base fluid. Lower operating temperatures lead to lower improvements. This makes sense to the hypothesis of the suitability of nanofluids for PTC applications involving high temperatures. This result is supported by the behavior of the Figure of Merit (FoM) illustrated in Fig. 7. It is clear that in general the FoM is greater than 1 (except at very low concentrations at low operating temperatures). A maximum FoM of 1.9 is reached at a temperature of $650 \mathrm{~K}$ and at a concentration of nanoparticles equal to $5 \%$.

Simulations were carried out to evidence the effect of using nanofluids in PTCs instead of the base fluid. The resolution of the governing equations has permitted to predict the temporary thermal behavior of the PTC. Considering the base fluid, a mass flow rate of $0.5 \mathrm{~kg} / \mathrm{s}$ and an inlet temperature of $323 \mathrm{~K}\left(50{ }^{\circ} \mathrm{C}\right)$, Fig. 8 shows the instantaneous variation of the fluid 
temperature along the day and along the axial direction of the PTC. As the working fluid flows inside the absorber, it gets gradually heated. The maximum temperature is reached at the outlet of the collector when the incident beam radiation is at its peak value (midday).

The next set of results illustrates the effect of using nanofluids as working fluids in the PTC. The same previous operating conditions were considered. The temporary evolution of the outlet temperature is depicted in Fig. 9. The nanoparticle concentration was set to a value of $\phi=3 \%$. One can see clearly that the nanofluids reach higher temperatures than the base fluid, especially at high radiation levels inducing greater heat propagation in the absorber and working fluid. $\mathrm{CuO}$ based nanofluid leads to the most significant increase in the outlet temperature while the other nanofluids give approximately the same thermal response with a little advantage of $\mathrm{TiO}_{2}$ based nanofluid. Based on this, the calculation of thermal efficiency and exergy efficiency was numerically investigated by evaluating the integrals expressions in Eqs. (30)-(31) using the trapezoidal method. The results are reported in Fig. 10 and Fig. 11, respectively.

Fig. 10 shows a minor improvement of the thermal efficiency of the PTC when nanofluids are used instead of the base fluid with no significant difference between the tested nanofluids. It is because the inlet temperature is fixed to $323 \mathrm{~K}$ which does not allow considerable improvements of the convective heat transfer coefficient $h_{f}$ as highlighted in Figs. 5 and 6. The enhancement of the exergy efficiency is more significant than the thermal efficiency (see Fig. 11). This result can be justified by the fact that the specific heat capacity of the nanofluid is considerably less important than the one of the base fluid which induces a more pronounced increase on the exergy output $\mathrm{E}_{\mathrm{u}}$ (see Eq. (31) and Fig. 3 (c)).

Fig. 12 shows the thermal efficiency and exergy efficiency plotted against the parameter $\left(T_{i n}-T_{a}\right) / G_{b t}$ supposing a constant inlet temperature of $323 \mathrm{~K}$ and a mass flow rate of $0.5 \mathrm{~kg} / \mathrm{s}$. It is shown that both thermal and exergy efficiencies follow a decreasing trend with respect to the defined ratio, with a sharper decrease for the thermal efficiency. For the base fluid, the maximum thermal efficiency is found to reach $65.7 \%$, while the minimum is about $43 \%$ with only a marginal benefit when using nanofluids. The exergy efficiency ranges between $3.05 \%$ and $8.5 \%$ for the base fluid case and gets improved more remarkably when nanofluids are employed. The peak exergy efficiency is attained by the $\mathrm{CuO}$ based nanofluid and is about $9.05 \%$. 
In order to evidence the combined effect of mass flow rate and inlet temperature, a parametric study was carried out comparing the energy and exergy efficiencies of the base fluid and $\mathrm{CuO}$ based nanofluid (as an example) for various conditions. This was made considering climatic conditions referring to the maximum solar radiation (observed at midday).

The results are plotted in Fig. 13 and Fig. 14. It is shown that, for the selected conditions, the thermal efficiency of the PTC follows a decreasing tendency with increasing inlet temperature independently of the working fluid nature. Increasing the mass flow rate generates a slight increase in the thermal efficiency. This increase is less important when the mass flow rate becomes higher. Comparing Fig. 13 (a) and Fig. 13 (b), one can remark that the presence of $\mathrm{CuO}$ nanoparticles in the base fluid enhances slightly the thermal efficiency, especially at higher temperatures.

From Fig. 14, it can be seen that the exergy efficiency increases as the inlet temperature increases, which is the opposite tendency for the thermal efficiency. Also, the mass flow rate impacts a little the exergy efficiency. The difference between the exergy efficiencies (base fluid and nanofluid) is also observed to be more important at increased inlet temperatures.

Relative daily energy gains associated with the use of nanofluids instead of the base fluid for various operating conditions in terms of mass flow rate, inlet temperature, nanoparticle type and concentration are given in Tables 4-5.

In Table 4, it is considered that the inlet temperature is set to a value of $323 \mathrm{~K}\left(50{ }^{\circ} \mathrm{C}\right)$. The observations that can be made are: (i) low concentrations of nanoparticles induce only minor improvements on the relative daily energy gains at high flow rates and are not advised at all for low flow rates; (ii) The nanoparticle type has a small effect of the gains with a certain advantage of $\mathrm{Al}_{2} \mathrm{O}_{3}$ nanoparticles; (iii) Increasing the mass flow rate has a minor positive effect of the relative daily energy gain.

Table 5 shows that increasing the inlet temperature generates a more considerable improvement of the relative daily energy gain. This is mainly due to the improvement occurring in the heat transfer coefficient at higher operating temperatures. From these two tables one can conclude that the best combination of mass flow rate and inlet temperature is when both are maximized. The maximum daily relative gain that can be reached is about 1.46 $\%$ by using $5 \%$ of $\mathrm{Al}_{2} \mathrm{O}_{3}$ in the base fluid. 
Another global conclusion that can be drawn is that operating conditions affect differently the energy and exergy related indicators, especially in terms of inlet temperature. Further detailed optimization should be conducted to ensure the best combination of design parameters selection based on the solar application.

\section{Conclusion}

A validated and detailed mathematical model was proposed to examine the benefits of using nanofluids as working fluids in parabolic trough collectors for medium and high temperature applications. Energy and exergy analyses were carried out based on real fluctuating operating conditions. Nanoparticles type and concentration, mass flow rate and inlet temperature were the parameters studied and the performance indices included the Figure of Merit, instantaneous outlet leaving the collector, thermal efficiency, exergy efficiency and relative gain in the thermal energy delivered to the utilization. The following conclusions have been made:

- Presence of nanoparticles in the base fluid enhances the convective heat transfer and can lead to higher values of the FoM. For Cuo based nanofluid, the FoM is greater than 1 for nanoparticle concentration $>1 \%$ and can exceed 1.8 at an operating temperature of $650 \mathrm{~K}$ and a nanoparticle concentration of $5 \%$.

- Nanofluids achieved higher temperatures than the base fluid, especially at higher levels of radiation. $\mathrm{CuO}$ based nanofluid leads to the most significant increase in the outlet temperature while the other nanofluids give approximately the same thermal behavior with a small advantage of $\mathrm{TiO}_{2}$ based nanofluid

- For a nanoparticle concentration of 3\%, only a minor improvement of the thermal efficiency of the PTC when nanofluids are used instead of the base fluid with no significant difference between the tested nanofluids.

- For similar conditions, the enhancement of the exergy efficiency is more significant than the thermal efficiency.

- The exergy efficiency varied between $3.05 \%$ and $8.5 \%$ for the base fluid case and gets improved more remarkably when nanofluids are employed. The peak exergy efficiency is attained by the $\mathrm{CuO}$ based nanofluid and is about $9.05 \%$.

- The maximum daily relative gain in terms of thermal energy delivered that is about $1.46 \%$ by using $5 \%$ of $\mathrm{Al}_{2} \mathrm{O}_{3}$ in the base fluid. 
- The parametric analysis showed that the operating conditions (i.e. mass flow rate and inlet temperature) should be carefully controlled for optimal energetic and exergetic performances. 


\section{References}

1. Pachauri, R. K., Allen, M. R., Barros, V. R., Broome, J., Cramer, W., Christ, R., ... \& Dubash, N. K. (2014). Climate change 2014: synthesis report. Contribution of Working Groups I, II and III to the fifth assessment report of the Intergovernmental Panel on Climate Change (p. 151). IPCC.

2. Liddle, B., \& Sadorsky, P. (2017). How much does increasing non-fossil fuels in electricity generation reduce carbon dioxide emissions?. Applied Energy, 197, 212221.

3. Wüstenhagen, R., \& Menichetti, E. (2012). Strategic choices for renewable energy investment: Conceptual framework and opportunities for further research. Energy Policy, 40, 1-10.

4. Solangi, K. H., Islam, M. R., Saidur, R., Rahim, N. A., \& Fayaz, H. (2011). A review on global solar energy policy. Renewable and sustainable energy reviews, 15(4), 2149-2163.

5. Michael, A., \& Heracleous, C. (2017). Assessment of natural lighting performance and visual comfort of educational architecture in Southern Europe: The case of typical educational school premises in Cyprus. Energy and Buildings, 140, 443-457.

6. Streicher, W. (2015). Solar thermal technologies for domestic hot water preparation and space heating. Renewable Heating and Cooling: Technologies and Applications, 9.

7. Allouhi, A., Jamil, A., Kousksou, T., El Rhafiki, T., Mourad, Y., \& Zeraouli, Y. (2015). Solar domestic heating water systems in Morocco: an energy analysis. Energy Conversion and Management, 92, 105-113.

8. Allouhi, A., Kousksou, T., Jamil, A., Bruel, P., Mourad, Y., \& Zeraouli, Y. (2015). Solar driven cooling systems: An updated review. Renewable and Sustainable Energy Reviews, 44, 159-181.

9. Kabeel, A. E., \& El-Agouz, S. A. (2011). Review of researches and developments on solar stills. Desalination, 276(1), 1-12.

10. Hogerwaard, J., Dincer, I., \& Naterer, G. F. (2017). Solar energy based integrated system for power generation, refrigeration and desalination. Applied Thermal Engineering, 121, 1059-1069.

11. Khan, J., \& Arsalan, M. H. (2016). Solar power technologies for sustainable electricity generation-A review. Renewable and Sustainable Energy Reviews, 55, 414-425. 
12. Fernández-García, A., Zarza, E., Valenzuela, L., \& Pérez, M. (2010). Parabolic-trough solar collectors and their applications. Renewable and Sustainable Energy Reviews, 14(7), 1695-1721.

13. Flueckiger, S. M., Iverson, B. D., Garimella, S. V., \& Pacheco, J. E. (2014). Systemlevel simulation of a solar power tower plant with thermocline thermal energy storage. Applied Energy, 113, 86-96.

14. Perini, S., Tonnellier, X., King, P., \& Sansom, C. (2017). Theoretical and experimental analysis of an innovative dual-axis tracking linear Fresnel lenses concentrated solar thermal collector. Solar Energy, 153, 679-690.

15. Ahmadi, M. H., Ahmadi, M. A., Mellit, A., Pourfayaz, F., \& Feidt, M. (2016). Thermodynamic analysis and multi objective optimization of performance of solar dish Stirling engine by the centrality of entransy and entropy generation. International Journal of Electrical Power \& Energy Systems, 78, 88-95.

16. Boukelia, T., \& Mecibah, M. S. (2013). Parabolic trough solar thermal power plant: Potential, and projects development in Algeria. Renewable and Sustainable Energy Reviews, 21, 288-297.

17. Kaygusuz, K. (2011). Prospect of concentrating solar power in Turkey: the sustainable future. Renewable and Sustainable Energy Reviews, 15(1), 808-814.

18. Kousksou, T., Allouhi, A., Belattar, M., Jamil, A., El Rhafiki, T., Arid, A., \& Zeraouli, Y. (2015). Renewable energy potential and national policy directions for sustainable development in Morocco. Renewable and Sustainable Energy Reviews, 47, 46-57.

19. Jaramillo, O. A., Borunda, M., Velazquez-Lucho, K. M., \& Robles, M. (2016). Parabolic trough solar collector for low enthalpy processes: An analysis of the efficiency enhancement by using twisted tape inserts. Renewable Energy, 93, 125-141.

20. Bortolato, M., Dugaria, S., \& Del Col, D. (2016). Experimental study of a parabolic trough solar collector with flat bar-and-plate absorber during direct steam generation. Energy, 116, 1039-1050.

21. Qiu, Y., Li, M. J., He, Y. L., \& Tao, W. Q. (2016). Thermal performance analysis of a parabolic trough solar collector using supercritical $\mathrm{CO} 2$ as heat transfer fluid under non-uniform solar flux. Applied Thermal Engineering, 115, 1255-1265

22. Sokhansefat, T., Kasaeian, A. B., \& Kowsary, F. (2014). Heat transfer enhancement in parabolic trough collector tube using Al2O3/synthetic voil nanofluid. Renewable and Sustainable Energy Reviews, 33, 636-644. 
23. Ghasemi, S. E., \& Ranjbar, A. A. (2016). Thermal performance analysis of solar parabolic trough collector using nanofluid as working fluid: a CFD modelling study. Journal of Molecular Liquids, 222, 159-166.

24. Mwesigye, A., Huan, Z., \& Meyer, J. P. (2016). Thermal performance and entropy generation analysis of a high concentration ratio parabolic trough solar collector with Cu-Therminol@ VP-1 nanofluid. Energy Conversion and Management, 120, 449-465.

25. Bellos, E., Tzivanidis, C., Antonopoulos, K. A., \& Gkinis, G. (2016). Thermal enhancement of solar parabolic trough collectors by using nanofluids and convergingdiverging absorber tube. Renewable Energy, 94, 213-222.

26. Wang, Y., Xu, J., Liu, Q., Chen, Y., \& Liu, H. (2016). Performance analysis of a parabolic trough solar collector using A12O3/synthetic oil nanofluid. Applied Thermal Engineering, 107, 469-478.

27. Coccia, G., Di Nicola, G., Colla, L., Fedele, L., \& Scattolini, M. (2016). Adoption of nanofluids in low-enthalpy parabolic trough solar collectors: Numerical simulation of the yearly yield. Energy Conversion and Management, 118, 306-319.

28. https://www.therminol.com/products/Therminol-VP1

29. Xuan, Y., \& Roetzel, W. (2000). Conceptions for heat transfer correlation of nanofluids. International Journal of heat and Mass transfer, 43(19), 3701-3707.

30. Shahrul, I. M., Mahbubul, I. M., Khaleduzzaman, S. S., Saidur, R., \& Sabri, M. F. M. (2014). A comparative review on the specific heat of nanofluids for energy perspective. Renewable and Sustainable Energy Reviews, 38, 88-98.

31. Solangi, K. H., Kazi, S. N., Luhur, M. R., Badarudin, A., Amiri, A., Sadri, R., ... \& Teng, K. H. (2015). A comprehensive review of thermo-physical properties and convective heat transfer to nanofluids. Energy, 89, 1065-1086.

32. Kamyar, A., Saidur, R., \& Hasanuzzaman, M. (2012). Application of computational fluid dynamics (CFD) for nanofluids. International Journal of Heat and Mass Transfer, 55(15), 4104-4115.

33. Faizal, M., Saidur, R., Mekhilef, S., \& Alim, M. A. (2013). Energy, economic and environmental analysis of metal oxides nanofluid for flat-plate solar collector. Energy Conversion and Management, 76, 162-168.

34. Kalogirou, S. A. (2013). Solar energy engineering: processes and systems. Academic Press. 
35. Allouhi, A., Amine, M. B., Kousksou, T., Jamil, A., \& Lahrech, K. (2018). Yearly performance of low-enthalpy parabolic trough collectors in MENA region according to different sun-tracking strategies. Applied Thermal Engineering, 128, 1404-1419

36. Mokheimer, E. M., Dabwan, Y. N., Habib, M. A., Said, S. A., \& Al-Sulaiman, F. A. (2014). Techno-economic performance analysis of parabolic trough collector in Dhahran, Saudi Arabia. Energy Conversion and Management, 86, 622-633.

37. Shahin, M. S., Orhan, M. F., \& Uygul, F. (2016). Thermodynamic analysis of parabolic trough and heliostat field solar collectors integrated with a Rankine cycle for cogeneration of electricity and heat. Solar Energy, 136, 183-196.

38. Kalogirou, S. A. (2012). A detailed thermal model of a parabolic trough collector receiver. Energy, 48(1), 298-306.

39. Erdogan, A., Colpan, C. O., \& Cakici, D. M. (2017). Thermal design and analysis of a shell and tube heat exchanger integrating a geothermal based organic Rankine cycle and parabolic trough solar collectors. Renewable Energy, 109, 372-391.

40. Li, Q., Xuan, Y., \& Wang, J. (2003). Investigation on convective heat transfer and flow features of nanofluids. Journal of Heat transfer, 125(2003), 151-155.

41. Gómez-Villarejo, R., Martín, E. I., Navas, J., Sánchez-Coronilla, A., Aguilar, T., Gallardo et al. (2017). Ag-based nanofluidic system to enhance heat transfer fluids for concentrating solar power: Nano-level insights. Applied Energy, 194, 19-29.

42. Padilla, R. V., Fontalvo, A., Demirkaya, G., Martinez, A., \& Quiroga, A. G. (2014). Exergy analysis of parabolic trough solar receiver. Applied Thermal Engineering, 67(1), 579-586.

43. Petela, R. (2003). Exergy of undiluted thermal radiation. Solar Energy, 74(6), 469488.

44. Dudley, V., Kolb, G., Sloan, M., \& Kearney, D. (1994). SEGS LS2 solar collectorTest results. Report of Sandia National Laboratories, Report No. SANDIA94-1884. 


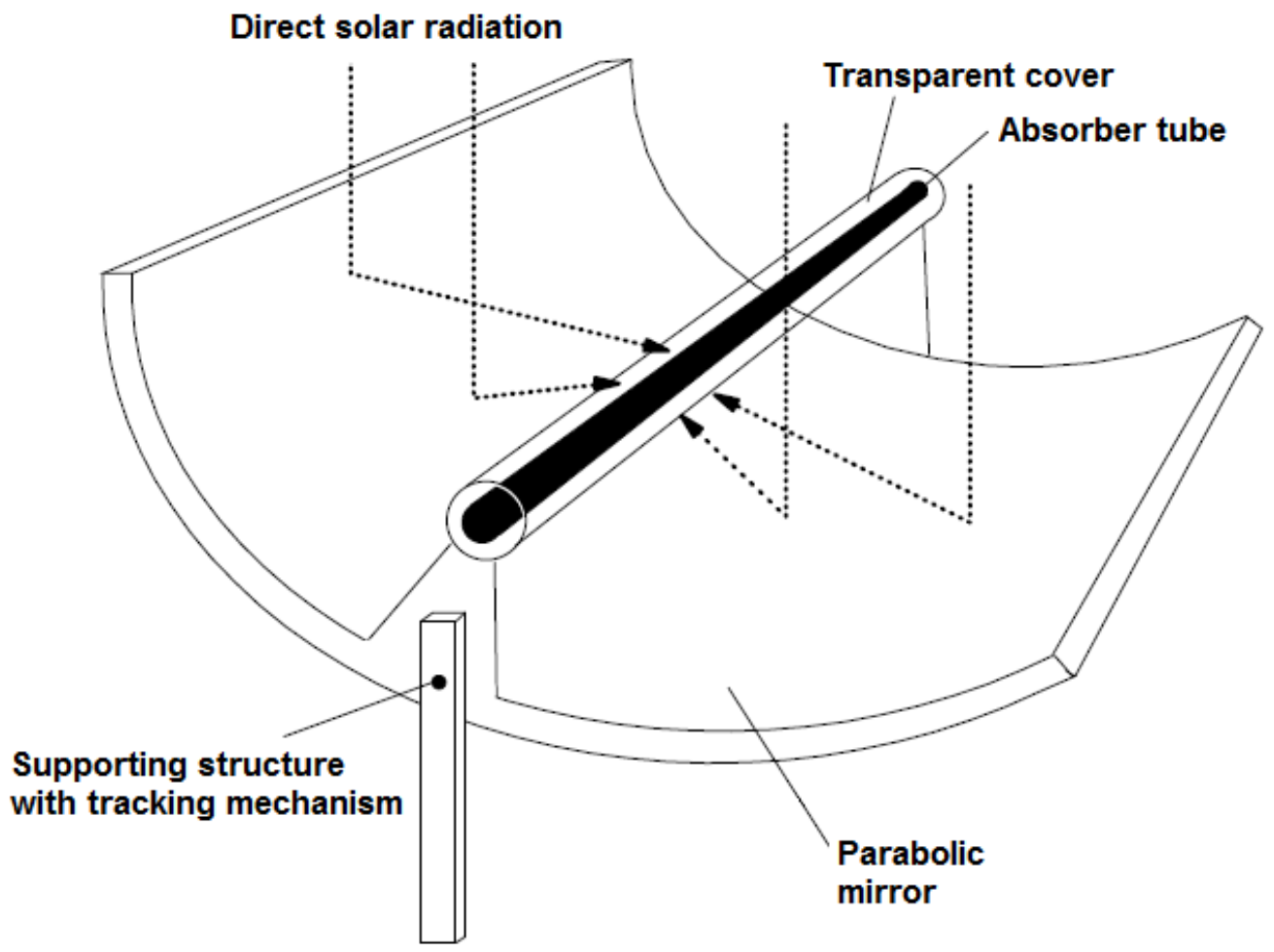




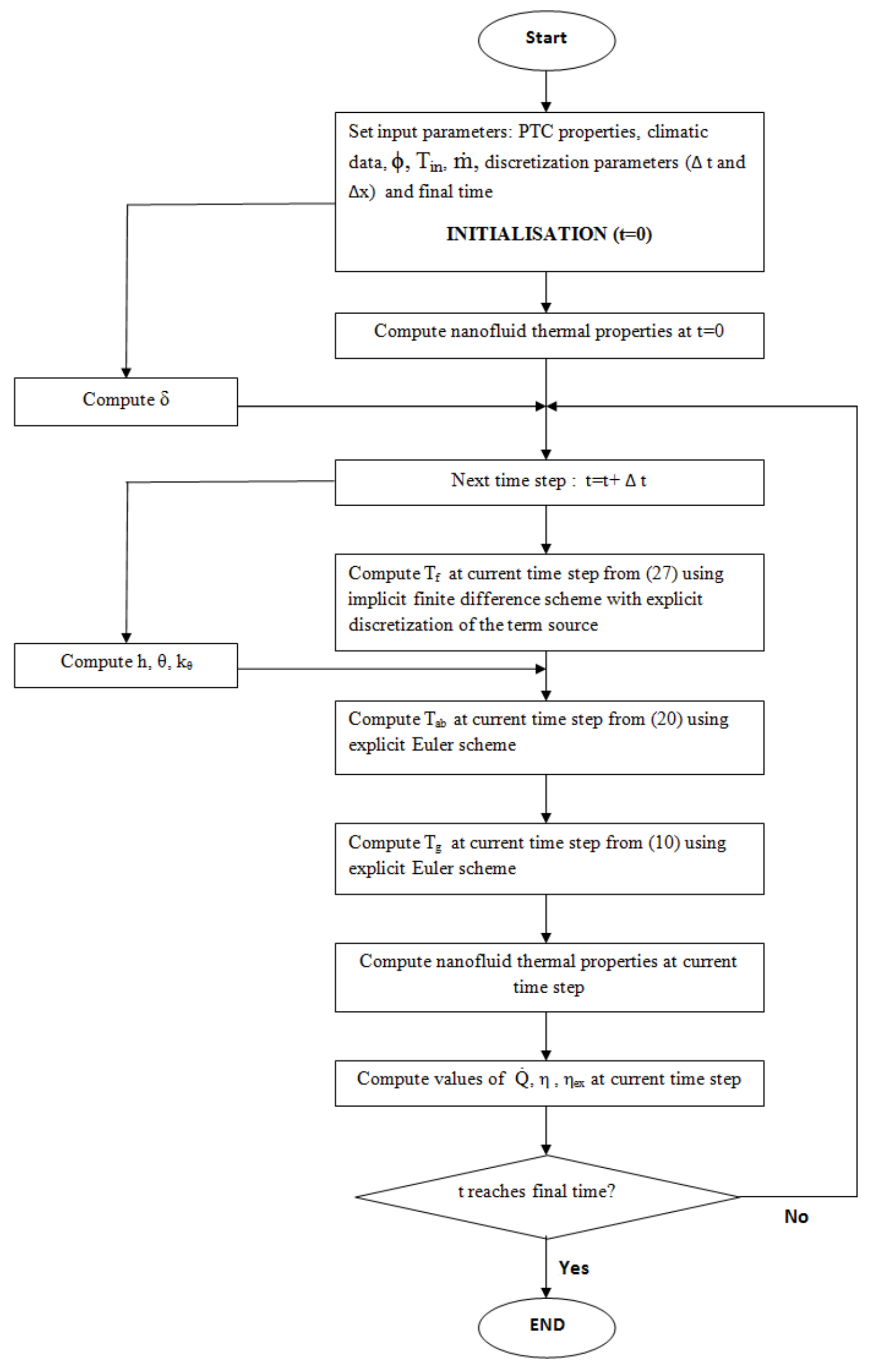




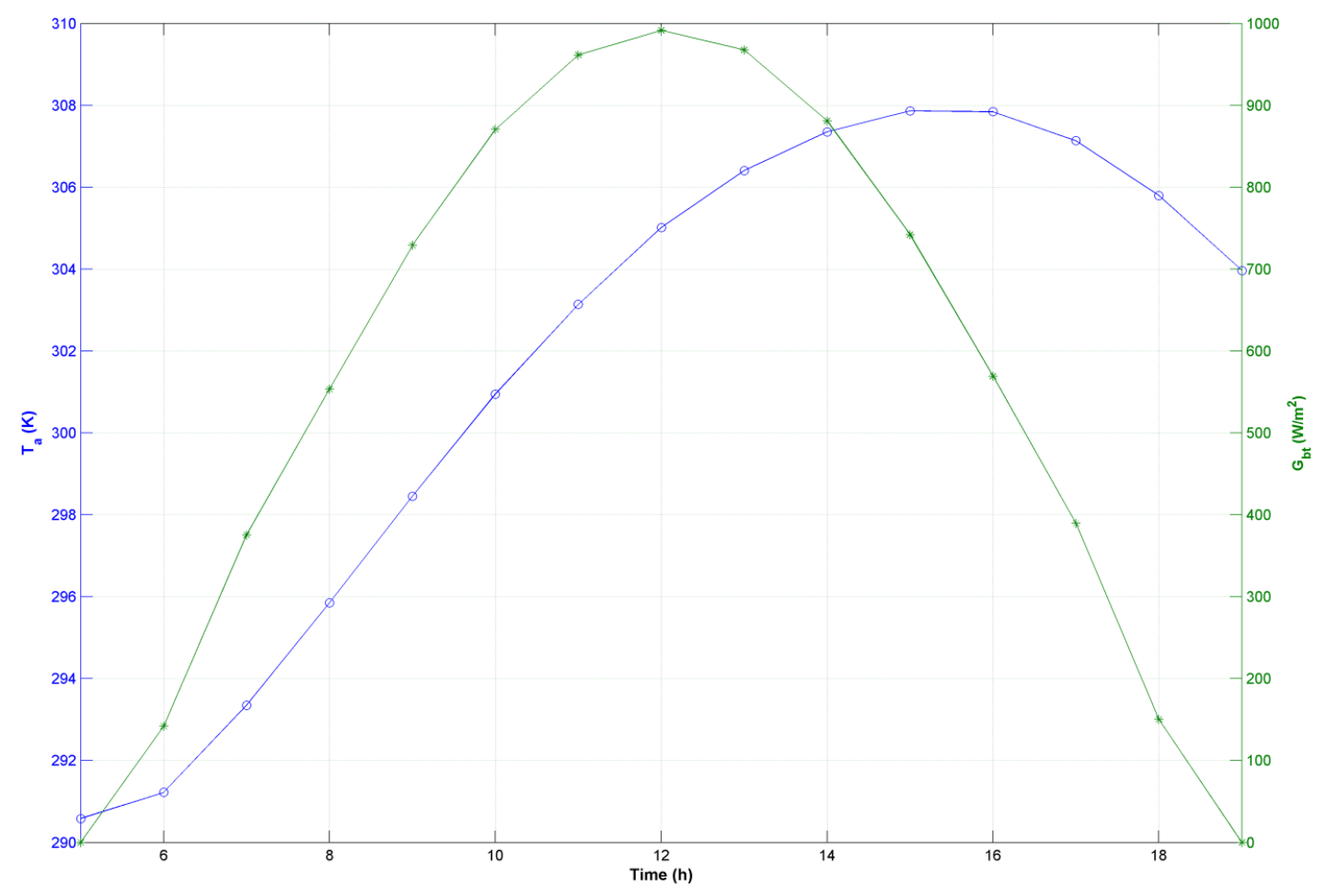

Fig. 3: Hourly variation of the ambient temperature (left axis) and beam incident 618 radiation (right axis)

619 


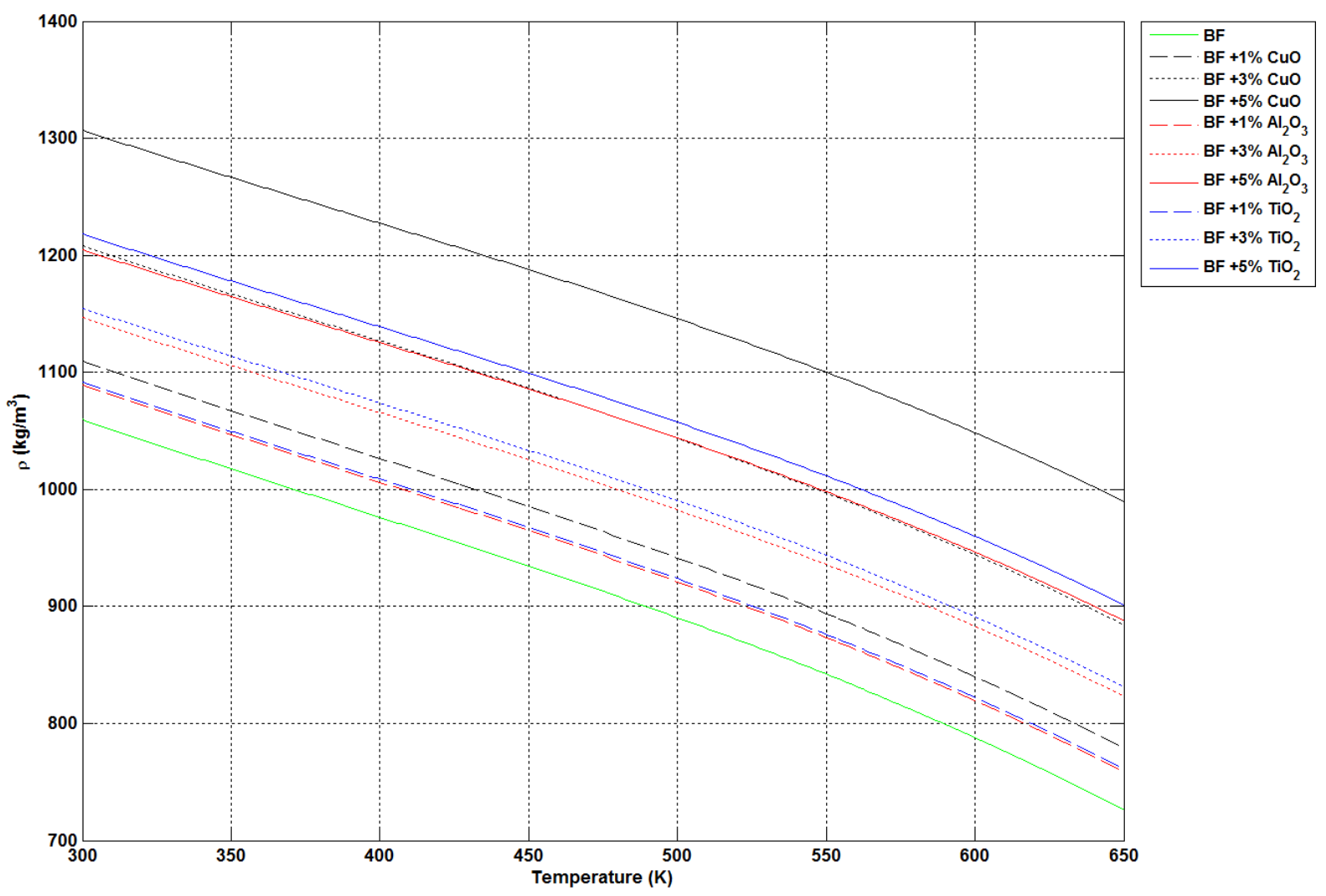




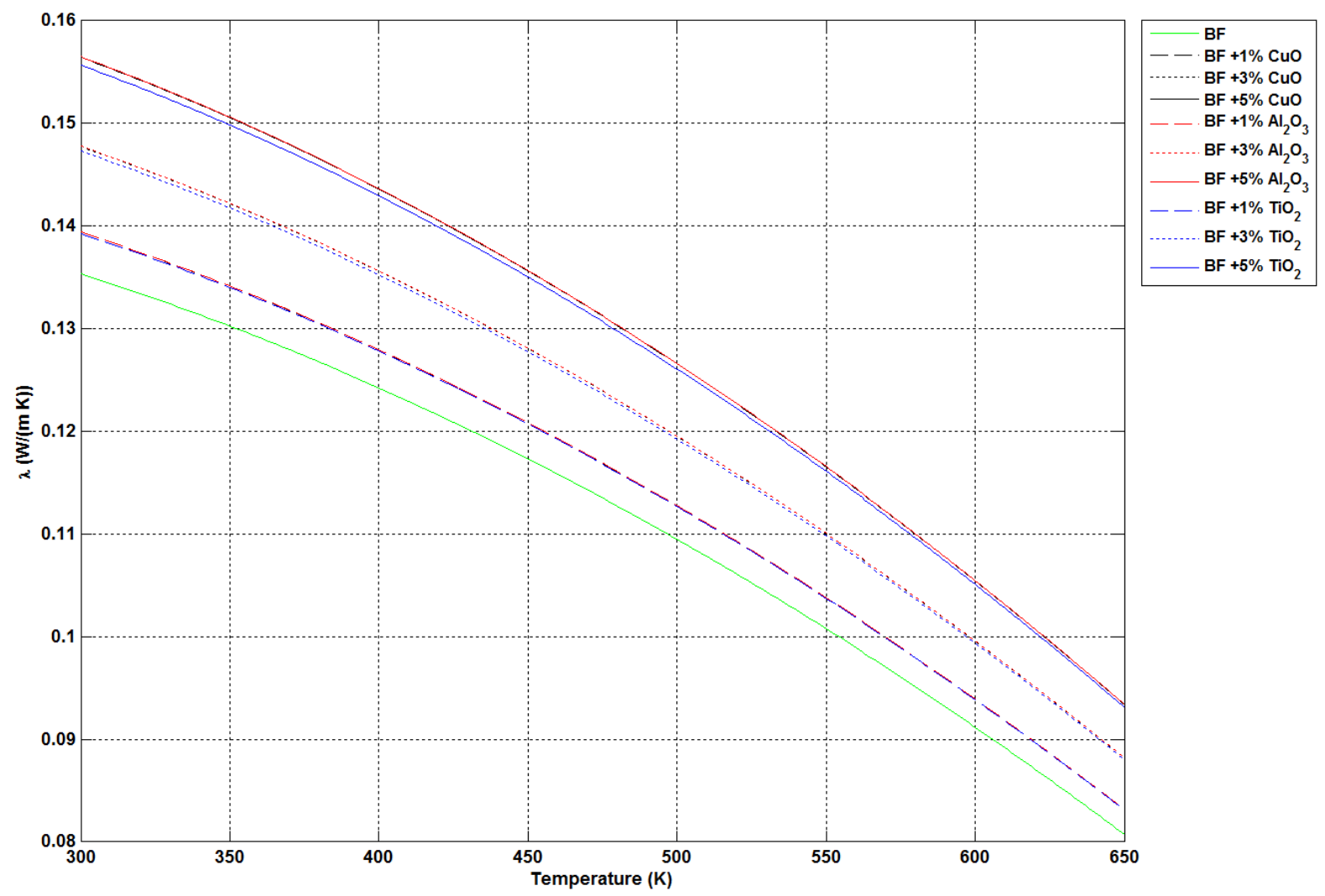


631

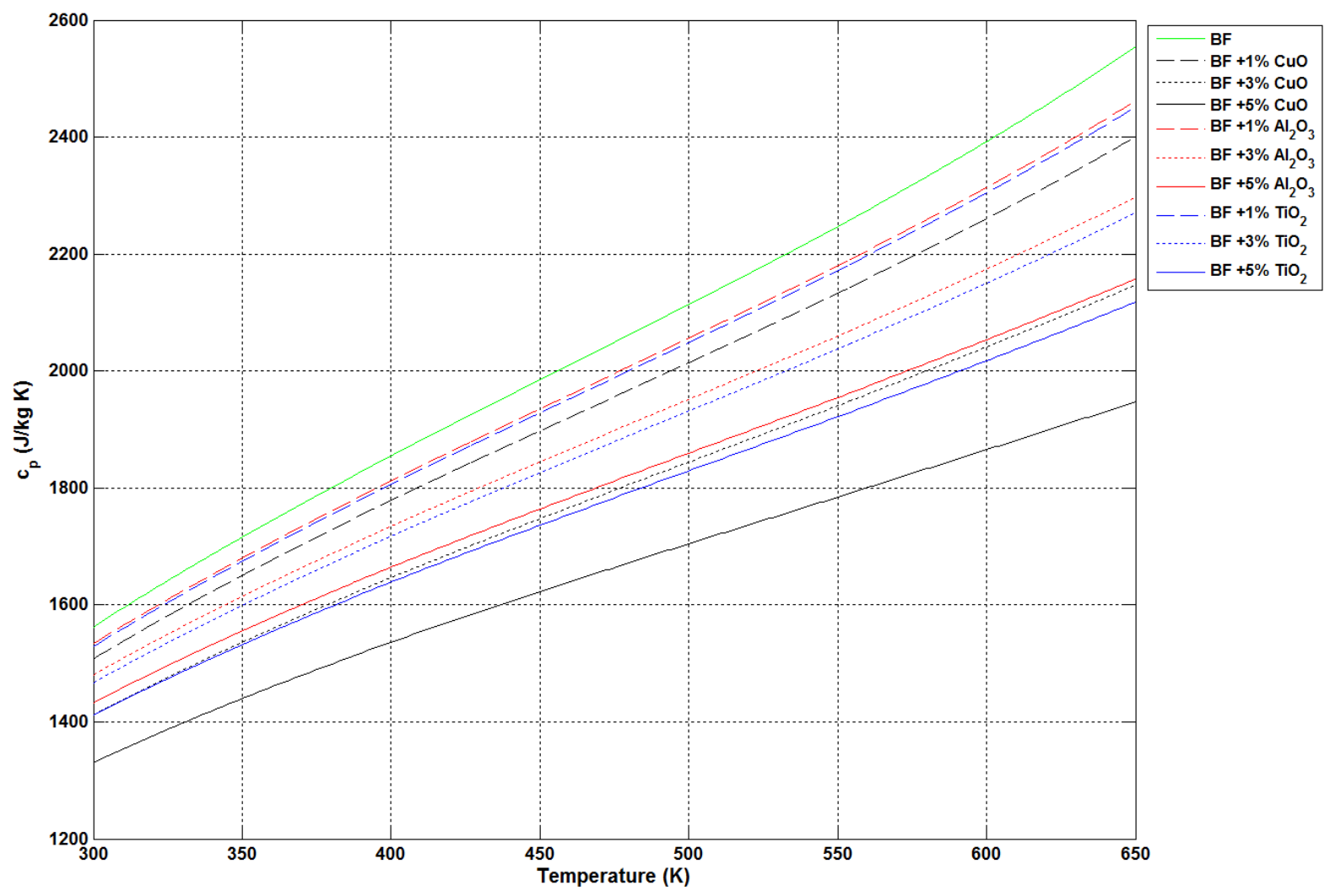

633

(c) Specific heat capacity 


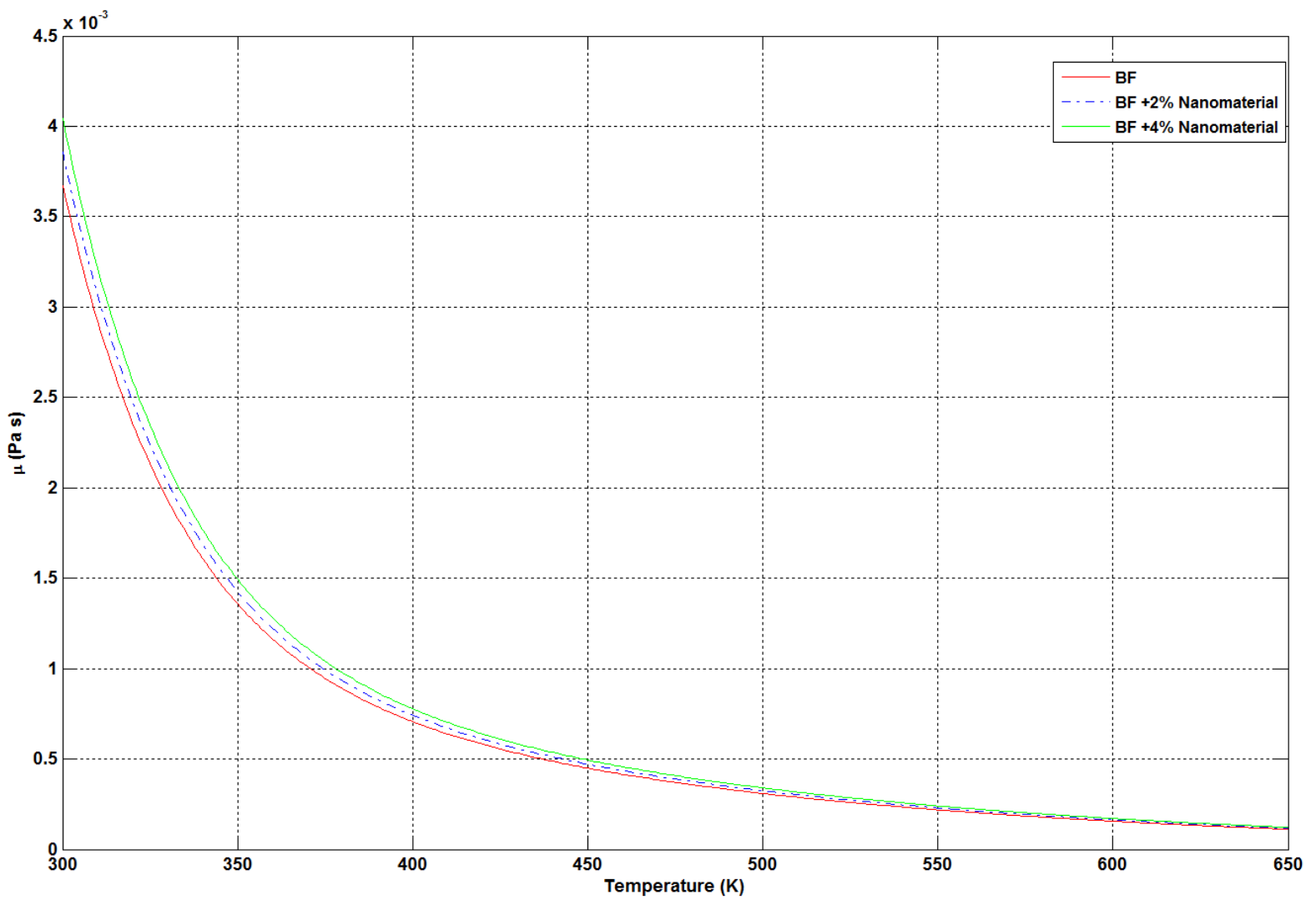

637

(d) Dynamic viscosity

638

639

640

Fig. 4: Thermal properties of base fluid and nanofluids

641 


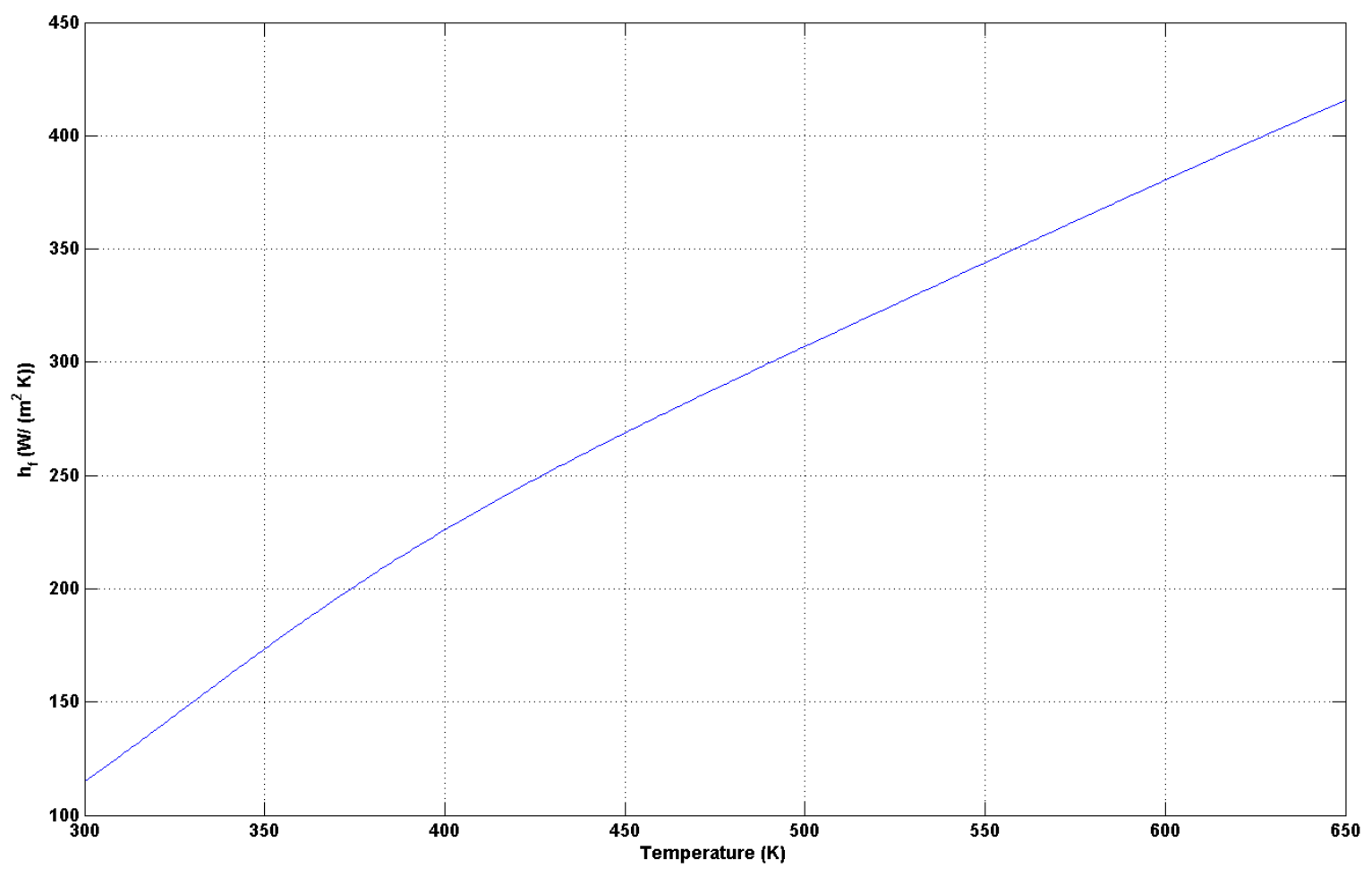

643 Fig. 5: Convective heat transfer coefficient for various fluid temperatures (base fluid)

644

645

646

647

648 


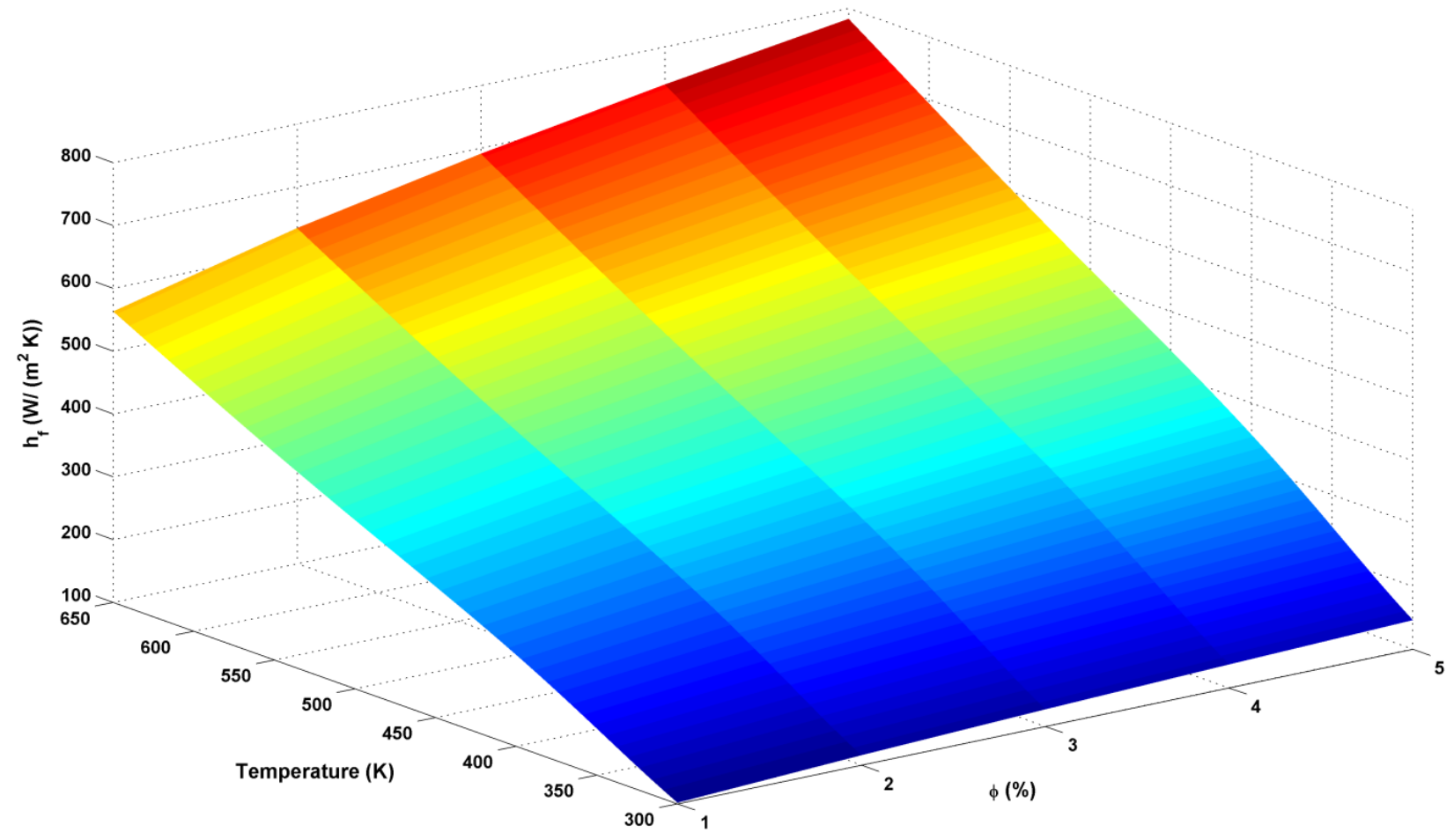

649

Fig. 6: Convective heat transfer coefficient for various fluid temperatures and 651 nanoparticle concentrations ( $\mathrm{CuO}$ based nanofluid)

652 


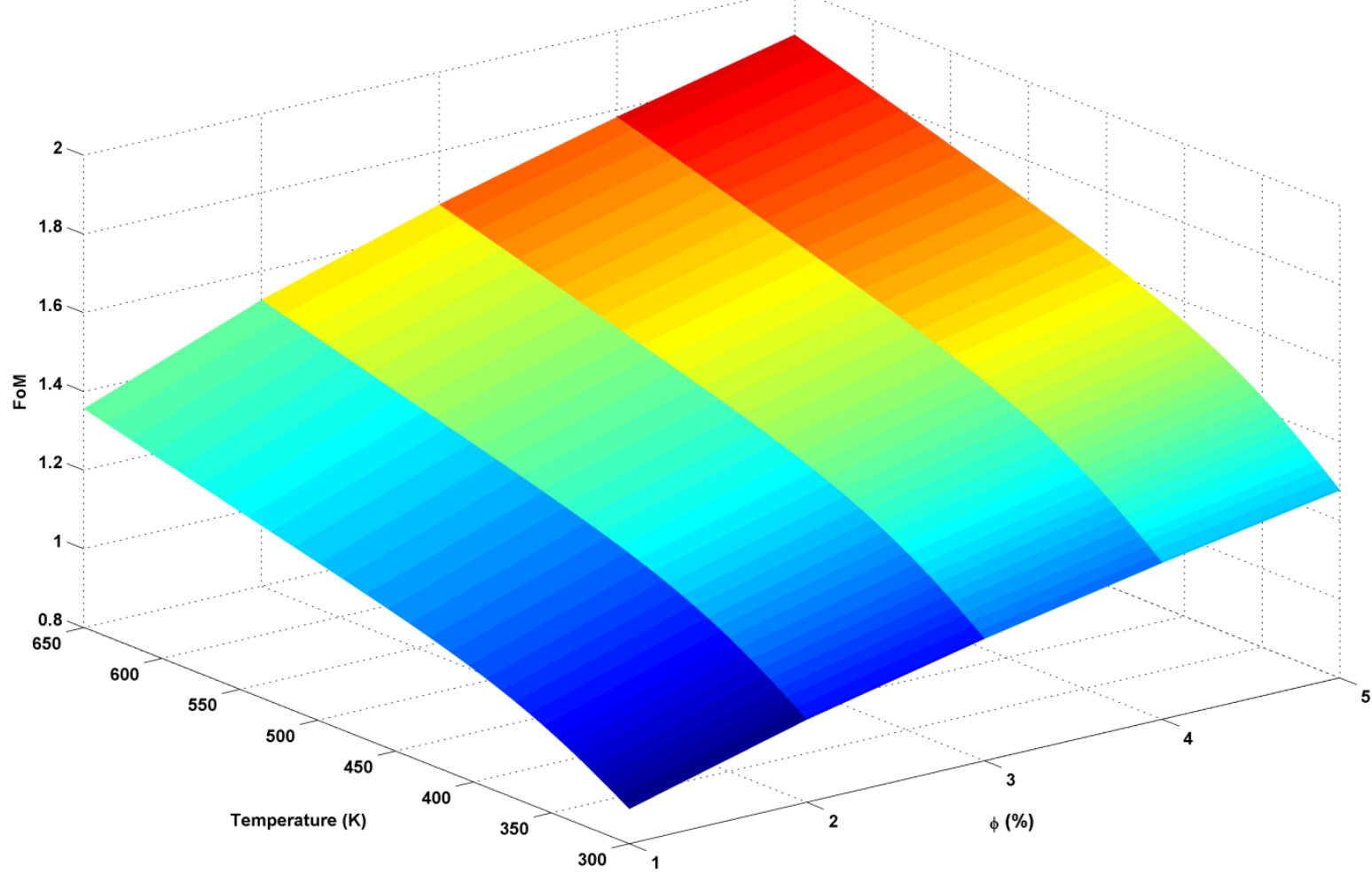

Fig. 7: Figure of Merit of Cuo based nanofluid for various fluid temperatures and 655 nanoparticle concentrations 


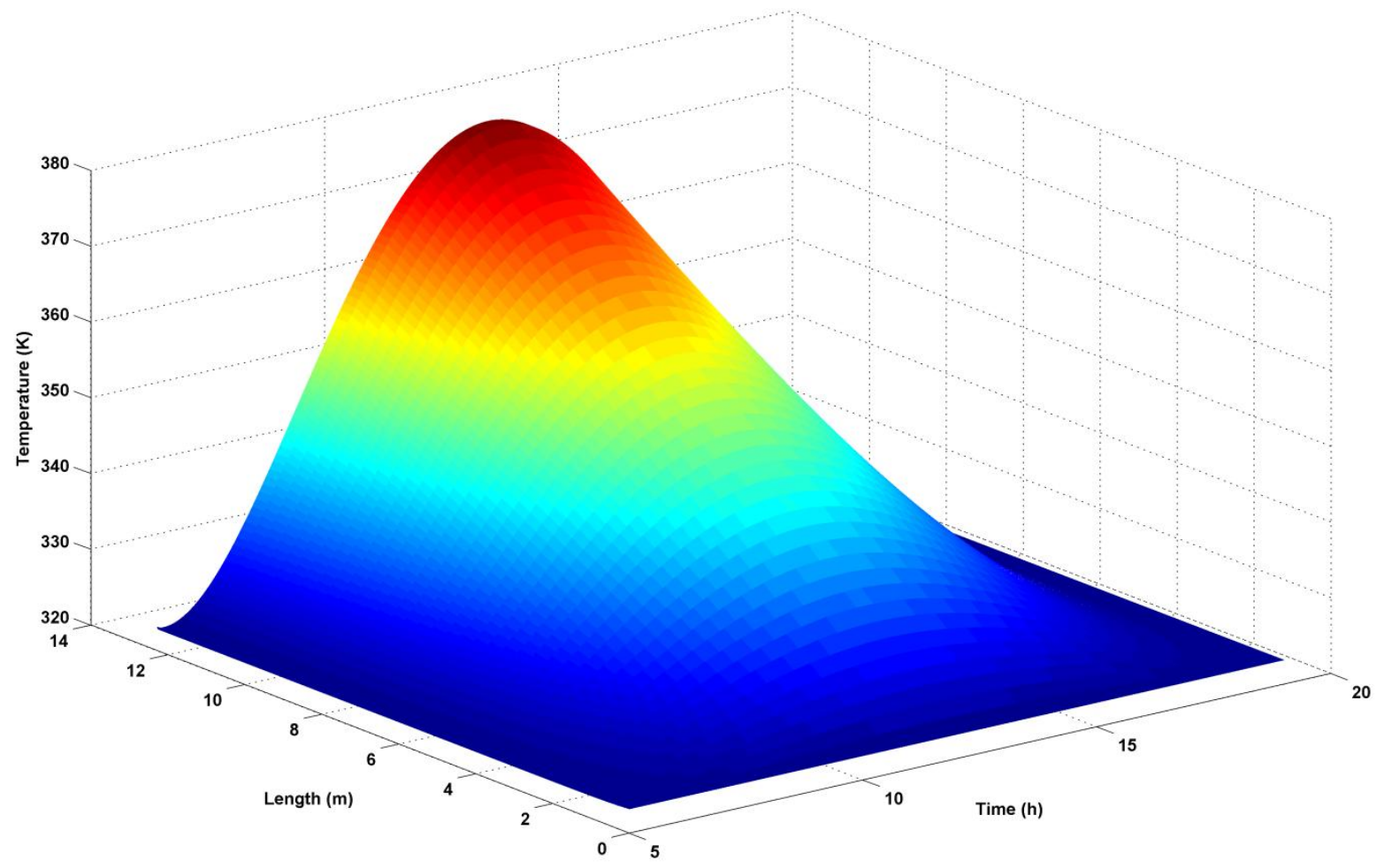

660 Fig. 8: Evolution of the base fluid temperature along the axial direction versus the time 


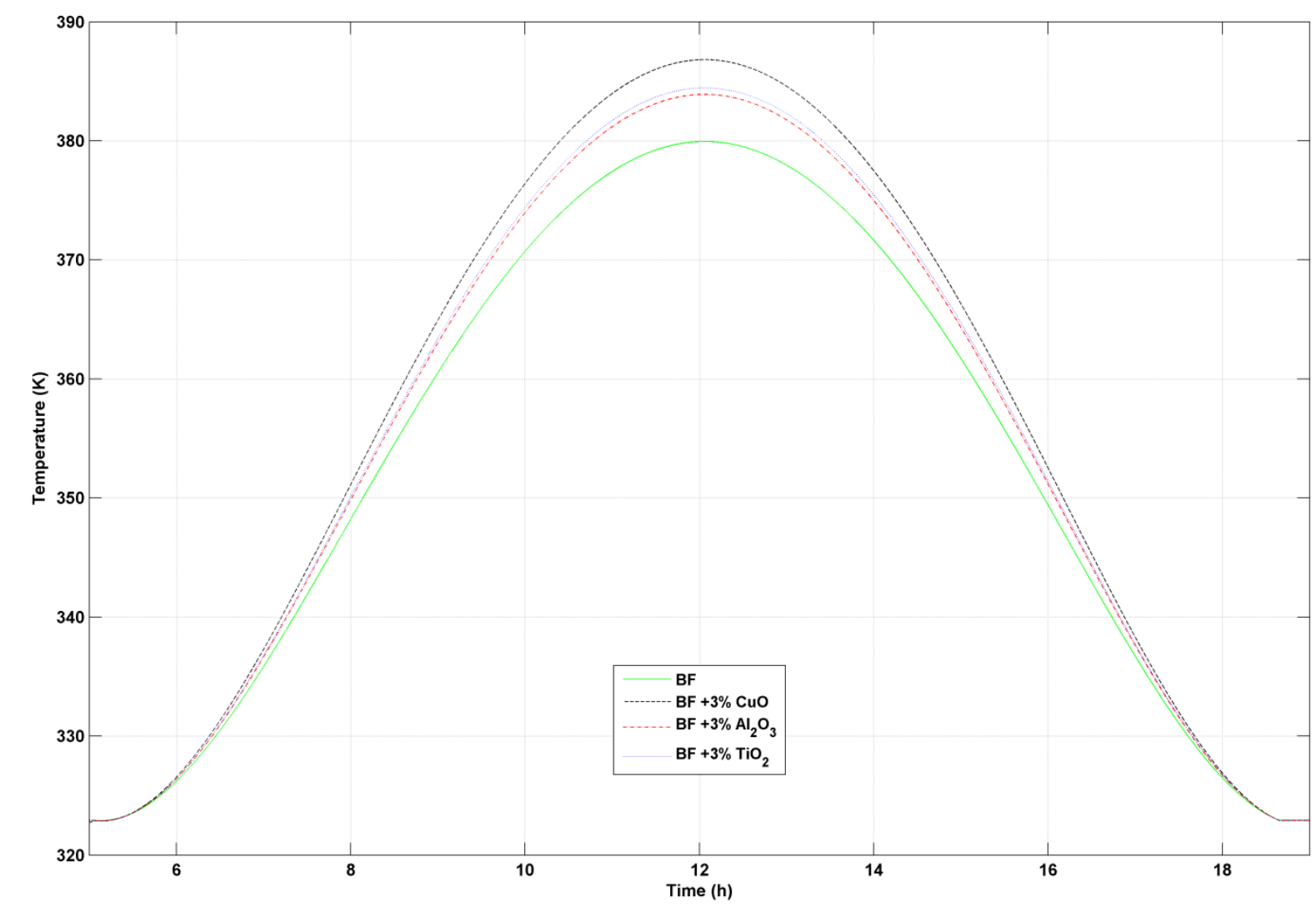

662

663 Fig. 9: Temporary evolution of outlet temperature of PTC (comparison between base 664 fluid and nanofluids) 
666

667 668

669

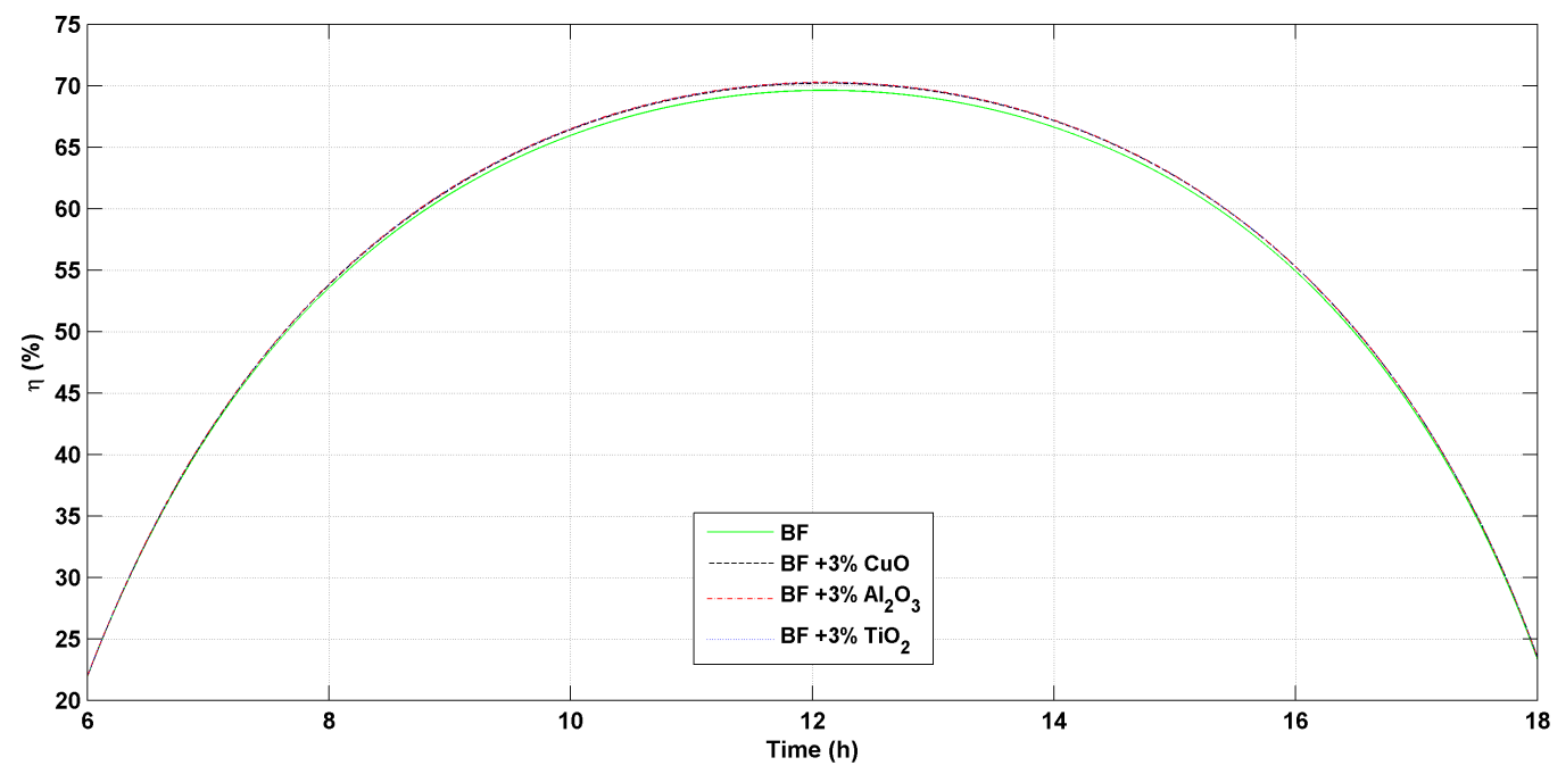

Fig. 10: Temporary evolution of thermal efficiency (comparison between base fluid and nanofluids) 
670

671

672

673

674

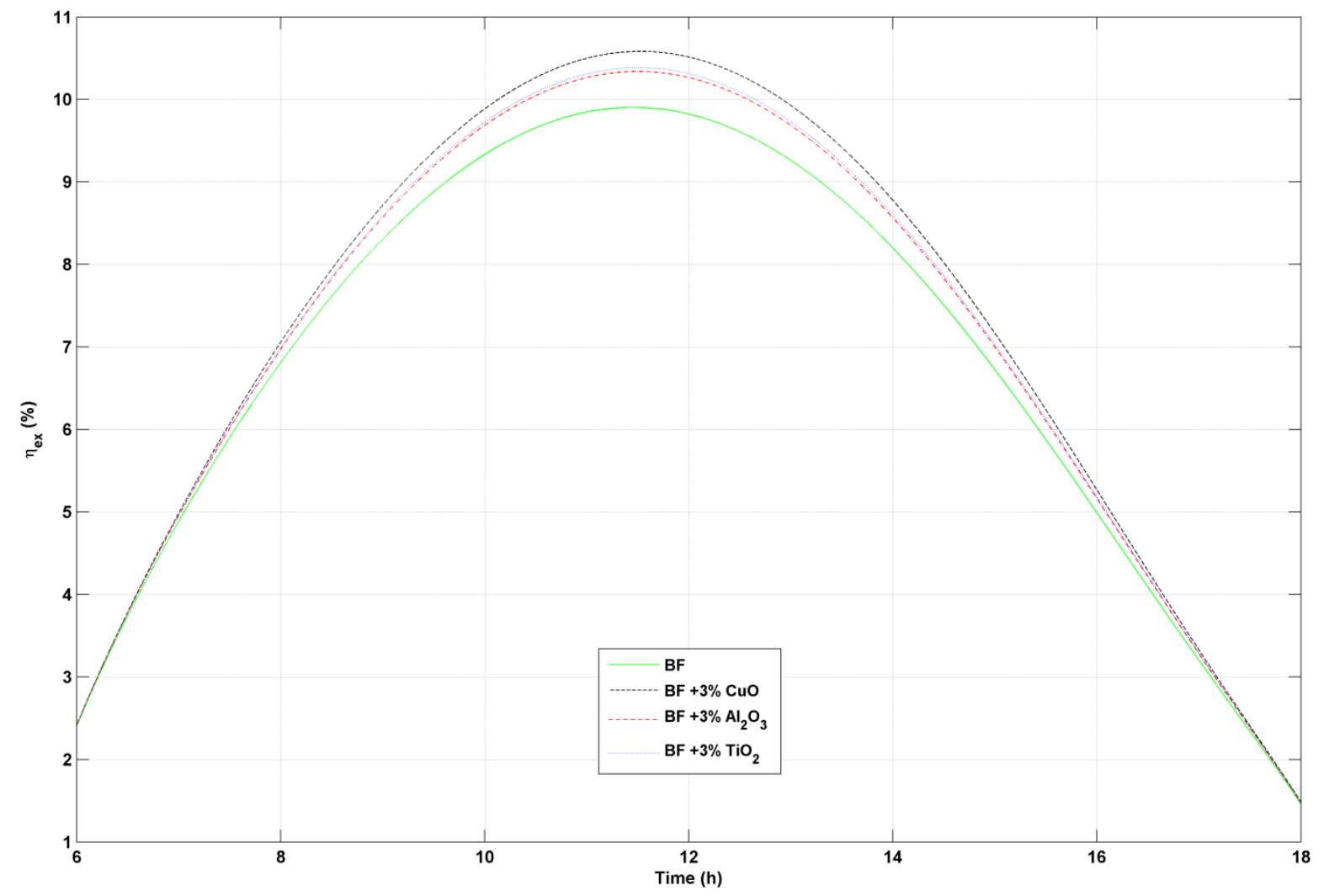

Fig. 11: Temporary evolution of exergy efficiency (comparison between base fluid and nanofluids) 


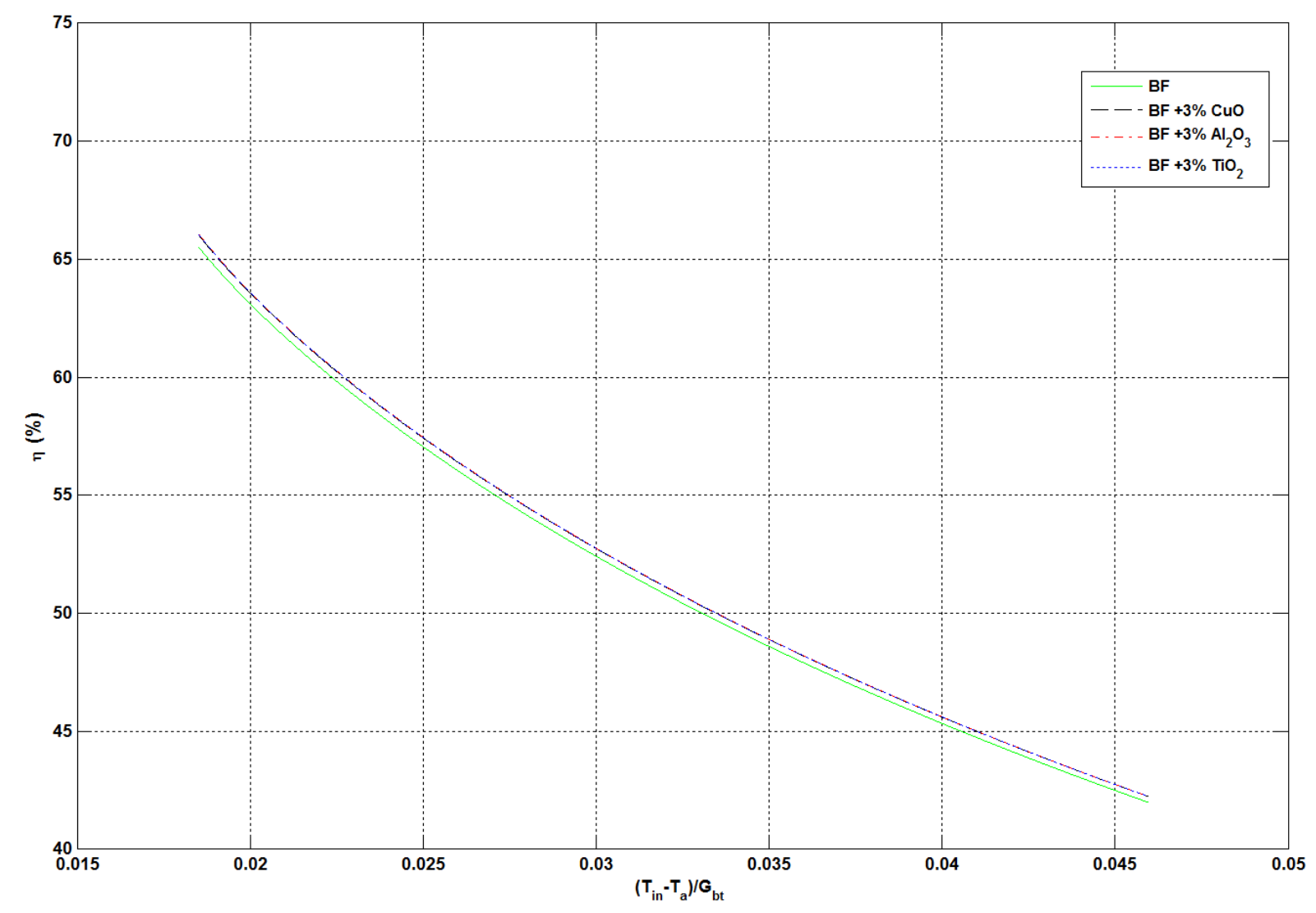

676

(a) Thermal efficiency

678 
680

681

682

683

684

685

686

687

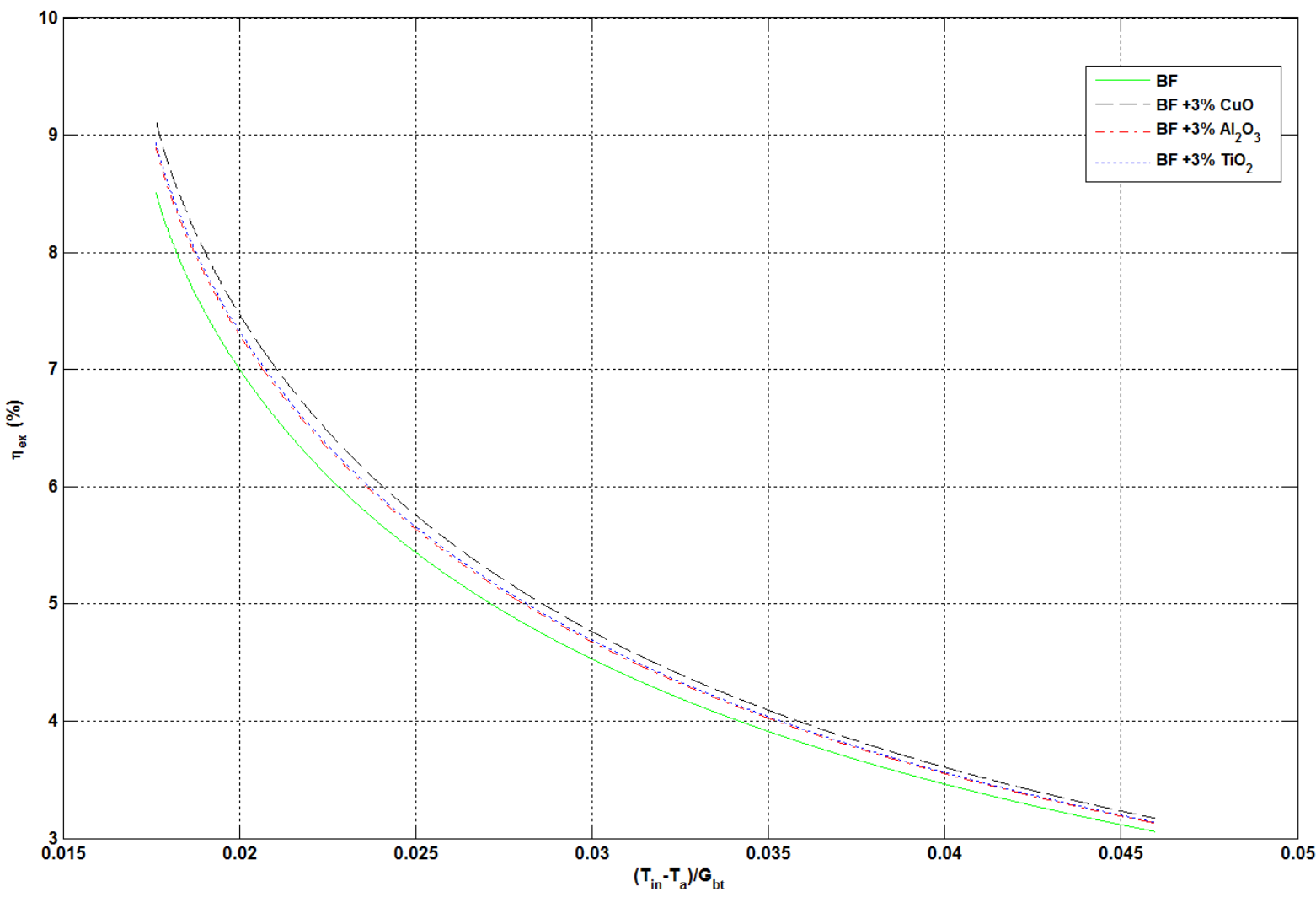

(b) Exergy efficiency

Fig. 12 : Comparison of thermal/exergy efficiency (base fluid and nanofluids) 


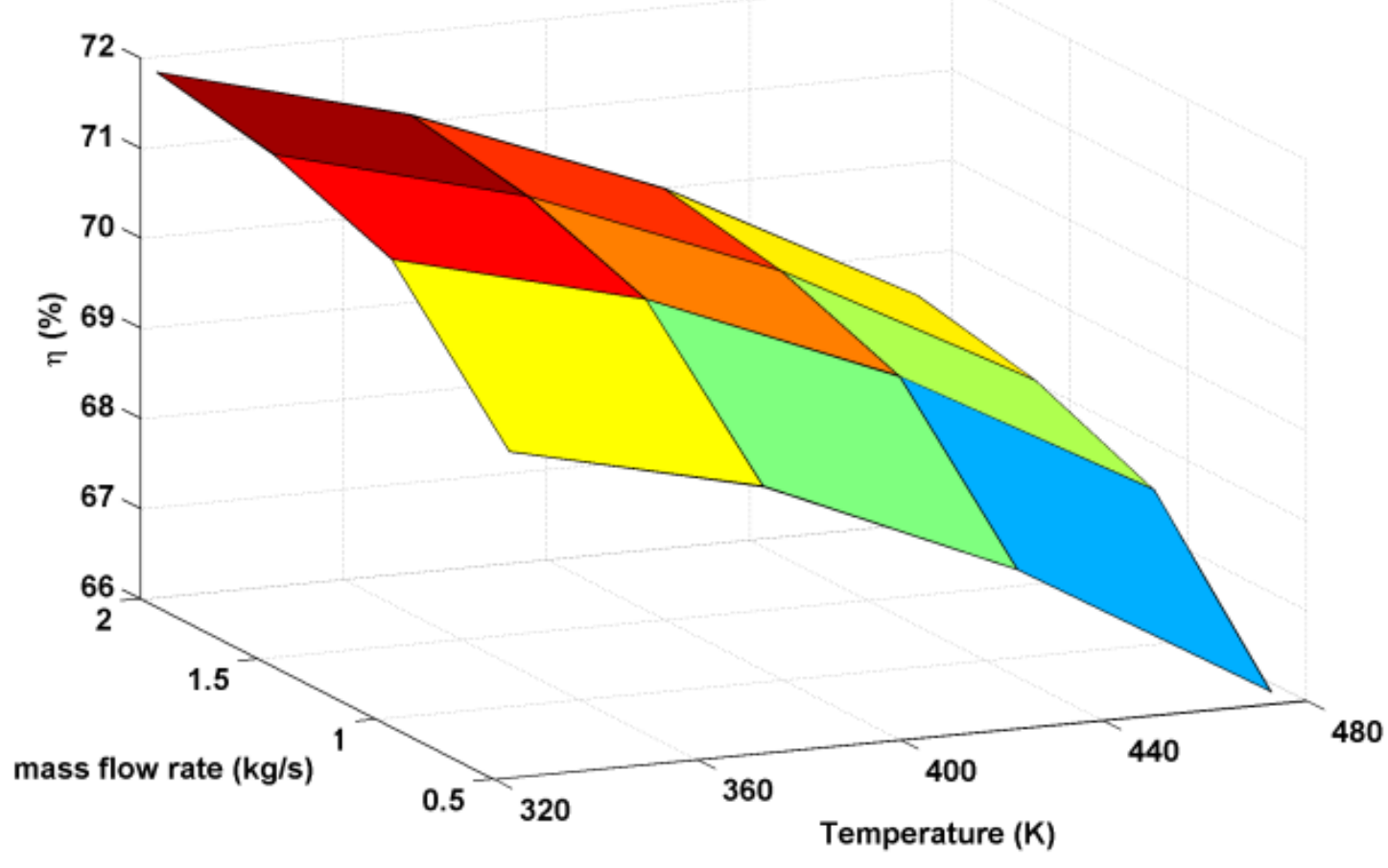

688

689

(a) Base fluid

690

691

692

693

694

695

696

697 


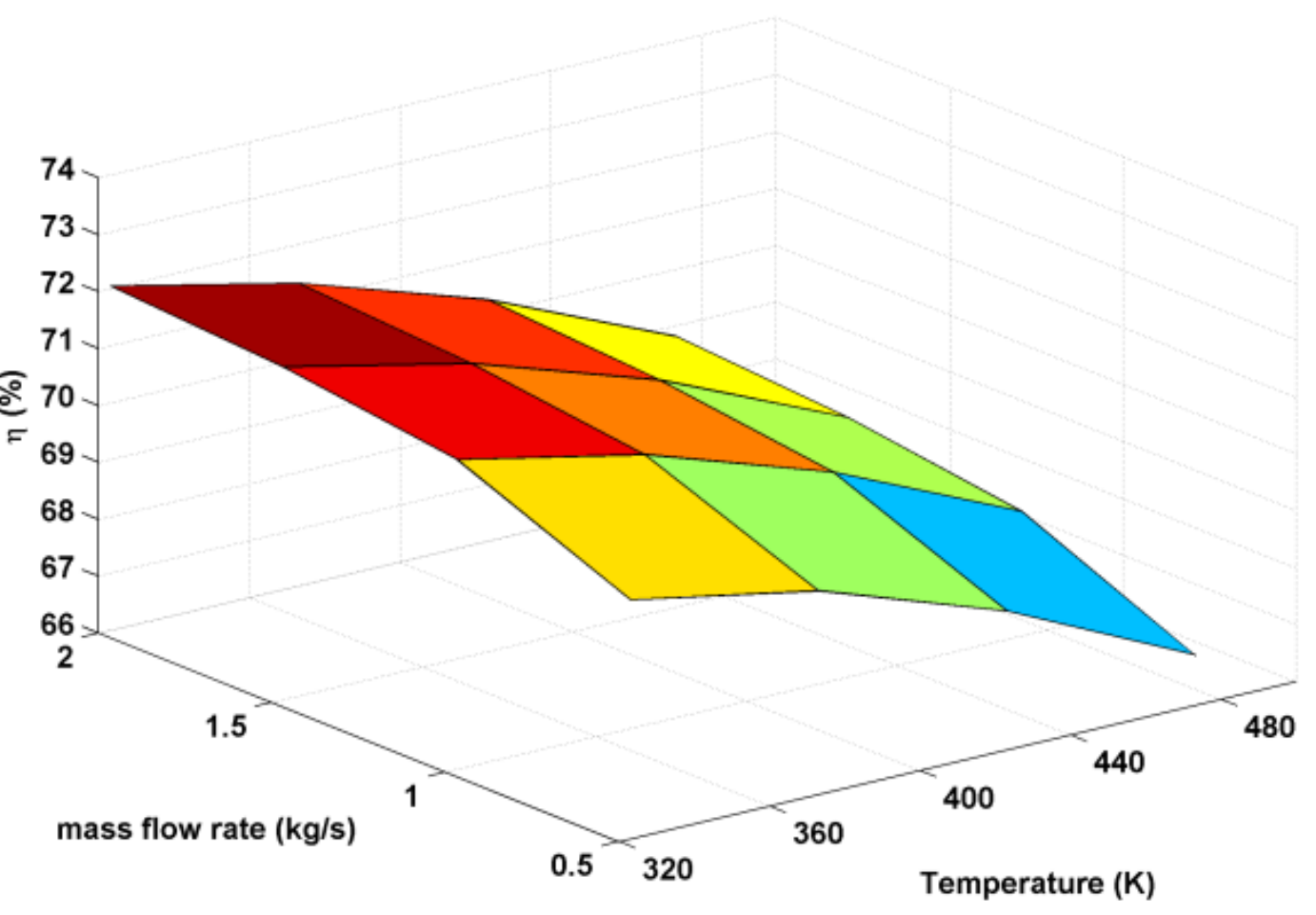

699

700

701

(b) CuO based nanofluid ( $\phi=3 \%$ )

702

703

704

705

706

Fig. 13: Thermal efficiency for various inlet temperatures and mass flow rates 


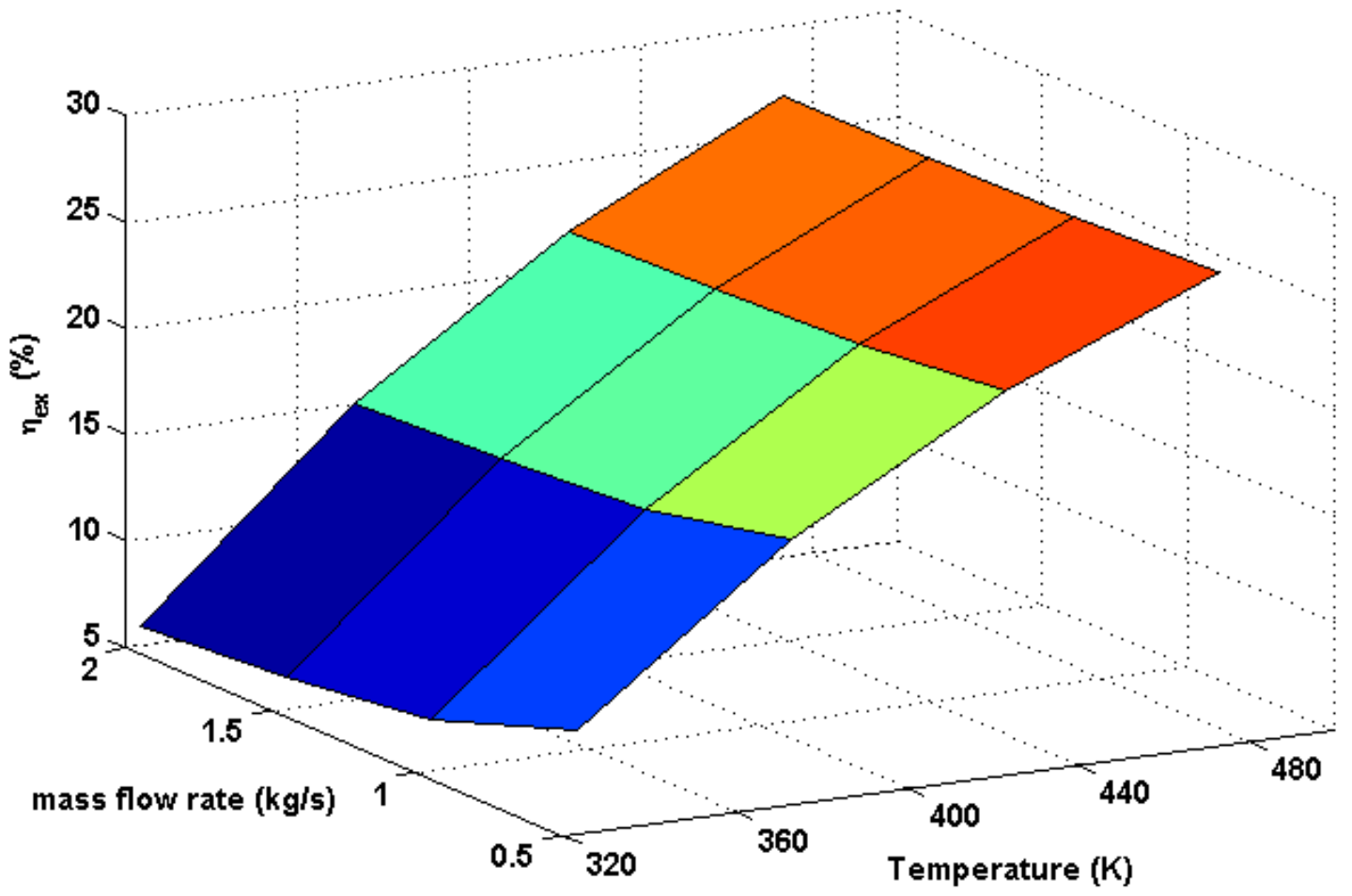

708

709

710

(a) Base fluid

711

712

713

714

715

716

717 


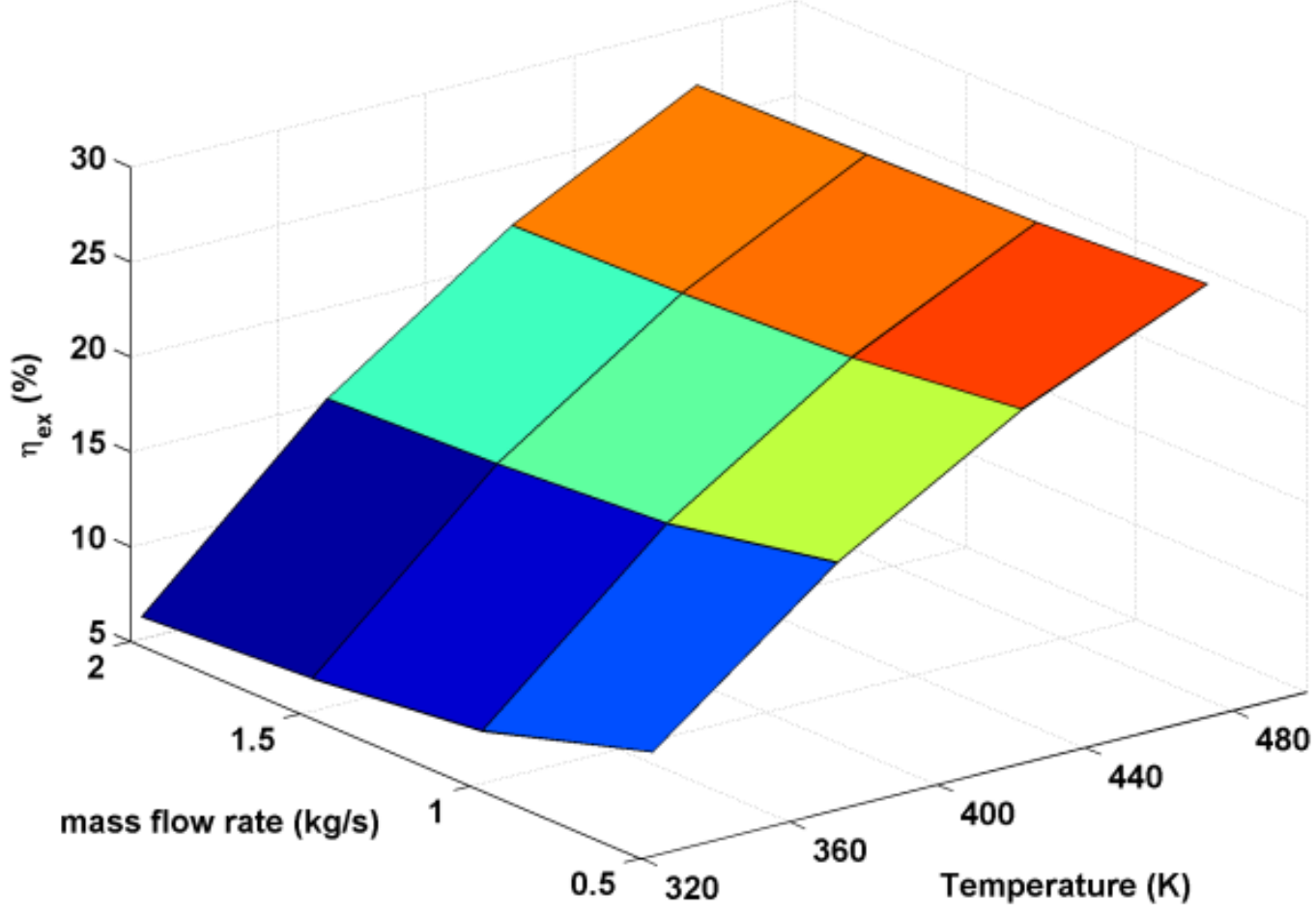

(b) CuO based nanofluid ( $\phi=3 \%)$

725 
Table 1: Properties of the used nanomaterials

\begin{tabular}{|l|l|l|l|}
\hline Material & $\begin{array}{l}\text { Specific heat } \\
(\mathbf{J} / \mathbf{k g ~ K})\end{array}$ & $\begin{array}{l}\text { Thermal conductivity } \\
(\mathbf{W} / \mathbf{m} \mathbf{~ K})\end{array}$ & Density $\left(\mathbf{k g} / \mathbf{m}^{\mathbf{3}}\right)$ \\
\hline Copper Oxide $(\mathrm{CuO})$ & 551 & 33 & 6000 \\
\hline Alumina $\left(\mathrm{Al}_{2} \mathrm{O}_{3}\right)$ & 773 & 40 & 3960 \\
\hline Titanium Oxide $\left(\mathrm{TiO}_{2}\right)$ & 692 & 8.4 & 4230 \\
\hline
\end{tabular}


Table 2: Geometrical and optical properties of PTC [37]

\begin{tabular}{|l|l|}
\hline Parameter & Value \\
\hline Length of the collector $(\mathrm{L})$ & $12.27 \mathrm{~m}$ \\
\hline Width of the collector & $5.76 \mathrm{~m}$ \\
\hline Receiver inner diameter & $0.066 \mathrm{~m}$ \\
\hline Receiver outer diameter & $0.07 \mathrm{~m}$ \\
\hline Glass envelope inner diameter & $0.115 \mathrm{~m}$ \\
\hline Glass envelope outer diameter & $0.121 \mathrm{~m}$ \\
\hline Absorptance of the receiver $(\alpha)$ & 0.96 \\
\hline Transmittance of the glass cover $(\tau)$ & 0.96 \\
\hline Reflectance of the mirror $\left(\mathrm{r}_{\mathrm{m}}\right)$ & 0.94 \\
\hline Intercept factor $(\gamma)$ & 0.867 \\
\hline
\end{tabular}

736 
Table 3: Comparison of model prediction with experimental tests from SNL [44]

\begin{tabular}{|c|c|c|c|c|c|c|c|c|c|c|c|}
\hline \multirow{2}{*}{ Test } & \multicolumn{5}{|c|}{ Test conditions } & \multicolumn{3}{|c|}{ Outlet Temperature (K) } & \multicolumn{3}{|c|}{ Thermal Efficiency (\%) } \\
\hline & $\mathrm{DNI}\left(\mathrm{W} / \mathrm{m}^{2}\right)$ & Wind $(\mathrm{m} / \mathrm{s})$ & $\mathrm{T}_{\mathrm{amb}}(\mathrm{K})$ & $\mathrm{T}_{\text {in }}(\mathrm{K})$ & \begin{tabular}{|l|}
$\begin{array}{l}\text { mass flow rate } \\
(\mathrm{kg} / \mathrm{s})\end{array}$ \\
\end{tabular} & SNL test & model & Deviation $(\mathrm{K})$ & SNL test & model & Deviation $(\%)$ \\
\hline State 1 & 933.7 & 2.60 & 294.35 & 375.35 & 0.66 & 397.15 & 397.08 & 0.07 & 72.51 & 69.61 & 2.9 \\
\hline State 2 & 968.2 & 3.70 & 295.55 & 424.15 & 0.68 & 446.45 & 446.07 & 0.38 & 70.9 & 69.84 & 1.06 \\
\hline State 3 & 937.9 & 1.00 & 301.95 & 570.95 & 0.61 & 590.05 & 590.88 & 0.83 & 67.98 & 66.64 & 1.34 \\
\hline
\end{tabular}

740 
Table 4: Relative energy gains using nanofluids instead of base fluid (effect of mass flow

\begin{tabular}{|c|c|c|c|c|c|c|c|c|}
\hline \multirow[b]{3}{*}{ HTF } & \multicolumn{8}{|c|}{ Mass flow rate $(\mathrm{kg} / \mathrm{s})$} \\
\hline & \multicolumn{2}{|c|}{0.5} & \multicolumn{2}{|l|}{1} & \multicolumn{2}{|c|}{1.5} & \multicolumn{2}{|l|}{2} \\
\hline & $\mathbf{Q}(\mathbf{k W h})$ & $\Delta \mathrm{e}(\%)$ & $\mathbf{Q}(\mathbf{k W h})$ & $\Delta \mathrm{e}(\%)$ & $\mathbf{Q}(\mathbf{k W h})$ & $\Delta \mathrm{e}(\%)$ & $\mathbf{Q}(\mathbf{k W h})$ & $\Delta \mathrm{e}(\%)$ \\
\hline $\mathrm{BF}$ & 358.4 & & 365.3 & & 367.7 & & 369 & \\
\hline $\mathrm{BF}+1 \% \mathrm{CuO}$ & 358.2 & -0.0558 & 365.7 & 0.1095 & 368.2 & 0.136 & 369.4 & 0.1084 \\
\hline $\mathrm{BF}+2 \% \mathrm{CuO}$ & 359.9 & 0.4185 & 366.5 & 0.3285 & 368.7 & 0.272 & 369.8 & 0.2168 \\
\hline $\mathrm{BF}+3 \% \mathrm{CuO}$ & 360.9 & 0.6975 & 367 & 0.4654 & 369 & 0.3535 & 370 & 0.271 \\
\hline $\mathrm{BF}+4 \% \mathrm{CuO}$ & 361.7 & 0.9208 & 367.4 & 0.5749 & 369.3 & 0.4351 & 370.2 & 0.3252 \\
\hline $\mathrm{BF}+5 \% \mathrm{CuO}$ & 362.2 & 1.0603 & 367.6 & 0.6296 & 369.4 & 0.4623 & 370.3 & 0.3523 \\
\hline $\mathrm{BF}+1 \% \mathrm{Al}_{2} \mathrm{O}_{3}$ & 358.4 & -0.0088 & 365.8 & 0.1369 & 368.2 & 0.136 & 369.5 & 0.1355 \\
\hline $\mathrm{BF}+2 \% \mathrm{Al}_{2} \mathrm{O}_{3}$ & 360.1 & 0.4743 & 366.6 & 0.3559 & 368.8 & 0.2992 & 369.9 & 0.2439 \\
\hline $\mathrm{BF}+3 \% \mathrm{Al}_{2} \mathrm{O}_{3}$ & 361.3 & 0.8092 & 367.2 & 0.5201 & 369.1 & 0.3807 & 370.1 & 0.2981 \\
\hline $\mathrm{BF}+4 \% \mathrm{Al}_{2} \mathrm{O}_{3}$ & 362 & 1.0045 & 367.5 & 0.6022 & 369.4 & 0.4623 & 370.3 & 0.3523 \\
\hline $\mathrm{BF}+5 \% \mathrm{Al}_{2} \mathrm{O}_{3}$ & 362.6 & 1.1719 & 367.8 & 0.6844 & 369.6 & 0.5167 & 370.5 & 0.4065 \\
\hline $\mathrm{BF}+1 \% \mathrm{TiO}_{2}$ & 358.3 & -0.0279 & 365.8 & 0.1369 & 368.2 & 0.136 & 369.4 & 0.1084 \\
\hline $\mathrm{BF}+2 \% \mathrm{TiO}_{2}$ & 360.1 & 0.4743 & 366.6 & 0.3559 & 368.8 & 0.2992 & 369.8 & 0.2168 \\
\hline $\mathrm{BF}+3 \% \mathrm{TiO}_{2}$ & 361.2 & 0.7813 & 367.1 & 0.4927 & 369.1 & 0.3807 & 370.1 & 0.2981 \\
\hline $\mathrm{BF}+4 \% \mathrm{TiO}_{2}$ & 361.9 & 0.9766 & 367.5 & 0.6022 & 369.4 & 0.4623 & 370.3 & 0.3523 \\
\hline $\mathrm{BF}+5 \% \mathrm{TiO}_{2}$ & 362.5 & 1.144 & 367.8 & 0.6844 & 369.5 & 0.4895 & 370.4 & 0.3794 \\
\hline
\end{tabular}




\begin{tabular}{|c|c|c|c|c|c|c|c|c|}
\hline \multirow{3}{*}{ HTF } & \multicolumn{8}{|c|}{ Inlet Temperature (K) } \\
\hline & \multicolumn{2}{|l|}{323} & \multicolumn{2}{|l|}{373} & \multicolumn{2}{|l|}{423} & \multicolumn{2}{|l|}{473} \\
\hline & $\mathbf{Q}(\mathbf{k W h})$ & $\Delta \mathrm{e}(\%)$ & $\mathbf{Q}(\mathbf{k W h})$ & $\Delta \mathrm{e}(\%)$ & $\mathbf{Q}(\mathbf{k W h})$ & $\Delta \mathrm{e}(\%)$ & $\mathbf{Q}(\mathbf{k W h})$ & $\Delta \mathrm{e}(\%)$ \\
\hline$\overline{\mathrm{BF}}$ & 358.4 & & 351.9 & & 341.9 & & 328.9 & \\
\hline $\mathrm{BF}+1 \% \mathrm{CuO}$ & 358.2 & -0.0558 & 352.7 & 0.2273 & 343.3 & 0.4095 & 330.8 & 0.5777 \\
\hline $\mathrm{BF}+2 \% \mathrm{CuO}$ & 359.9 & 0.4185 & 353.9 & 0.5683 & 344.4 & 0.7312 & 331.8 & 0.8817 \\
\hline $\mathrm{BF}+3 \% \mathrm{CuO}$ & 360.9 & 0.6975 & 354.7 & 0.7957 & 345 & 0.9067 & 332.4 & 1.0642 \\
\hline $\mathrm{BF}+4 \% \mathrm{CuO}$ & 361.7 & 0.9208 & 355.2 & 0.9378 & 345.4 & 1.0237 & 332.8 & 1.1858 \\
\hline $\mathrm{BF}+5 \% \mathrm{CuO}$ & 362.2 & 1.0603 & 355.5 & 1.023 & 345.7 & 1.1114 & 333 & 1.2466 \\
\hline $\mathrm{BF}+1 \% \mathrm{Al}_{2} \mathrm{O}_{3}$ & 358.4 & $\begin{array}{c}-0.0088 \\
\end{array}$ & 352.8 & 0.2558 & 343.4 & 0.4387 & 330.9 & 0.6081 \\
\hline $\mathrm{BF}+2 \% \mathrm{Al}_{2} \mathrm{O}_{3}$ & 360.1 & 0.4743 & 354.1 & 0.6252 & 344.6 & 0.7897 & 332.1 & 0.9729 \\
\hline $\mathrm{BF}+3 \% \mathrm{Al}_{2} \mathrm{O}_{3}$ & 361.3 & 0.8092 & 355 & 0.8809 & 345.4 & 1.0237 & 332.8 & 1.1858 \\
\hline $\mathrm{BF}+4 \% \mathrm{Al}_{2} \mathrm{O}_{3}$ & 362 & 1.0045 & 355.6 & 1.0514 & 345.9 & 1.1699 & 333.3 & 1.3378 \\
\hline $\mathrm{BF}+5 \% \mathrm{Al}_{2} \mathrm{O}_{3}$ & 362.6 & 1.1719 & 356 & 1.1651 & 346.3 & 1.2869 & 333.7 & 1.4594 \\
\hline $\mathrm{BF}+1 \% \mathrm{TiO}_{2}$ & 358.3 & -0.0279 & 352.8 & 0.2558 & 343.4 & 0.4387 & 330.9 & 0.6081 \\
\hline $\mathrm{BF}+2 \% \mathrm{TiO}_{2}$ & 360.1 & 0.4743 & 354.1 & 0.6252 & 344.6 & 0.7897 & 332 & 0.9425 \\
\hline $\mathrm{BF}+3 \% \mathrm{TiO}_{2}$ & 361.2 & 0.7813 & 354.9 & 0.8525 & 345.3 & 0.9944 & 332.7 & 1.1554 \\
\hline $\mathrm{BF}+4 \% \mathrm{TiO}_{2}$ & 361.9 & 0.9766 & 355.5 & 1.023 & 345.8 & 1.1407 & 333.2 & 1.3074 \\
\hline $\mathrm{BF}+5 \% \mathrm{TiO}_{2}$ & 362.5 & 1.144 & 355.9 & 1.1367 & 346.2 & 1.2577 & 333.5 & 1.3986 \\
\hline
\end{tabular}




\section{Response to Reviewers' comments}

To the editor,

Thank you for the opportunity given to revise our paper according to the pertinent comments of the reviewers. We inform you that we addressed all the comments raised and the changes were endorsed in the green color in the revised manuscript. Please find the detailed answers to the reviewers' comments below.

Thank for reconsideration of this submission

\section{Reviewer 1:}

Comment:

Manuscript is written very well. Good Quality work.

\section{Answer 1}

Thank you for the positive feedback.

\section{Reviewer 2:}

\section{Comment 1}

Highlights were not provided.

\section{Answer 1}

Highlights are now provided. Please see the revised manuscript.

\section{Comment 2}

There is no Nomenclature (symbols, sub/superscripts, acronyms). 


\section{Answer 2}

A Nomenclature is now provided. Please see the revised manuscript.

\section{Comment 3}

It would have been interesting if the authors evaluated nanoparticles' thermal properties through experimental validation, and not with the proposed equations (I'm referring in particular to Equations (7) and (8)).

\section{Answer 3}

Agreed. It is better to use experimental properties of nanoparticles. But, as we did not perform experimentations, we used these equations that are widely used in the literature.

\section{Comment 4}

Line 168. I would say "The PTC is schematically reported in Fig. 1".

\section{Answer 4}

Agreed. It was corrected as per suggestion

\section{Comment 5}

Lines 172 - 174. I suggest that the authors report a table, or a flow diagram, showing the inputs, the outputs and the calculations operated by the model.

\section{Answer 5}

A diagram was added as per suggestion. Please see the revised manuscript.

\section{Comment6}

Lines 185 - 186. Please specify that all the equations are referred to the length unit of the collector.

\section{Answer 6}

Agreed. It was specified as per suggestion

\section{Comment 7}


Equation (12). How was the incident angle modifier determined?

\section{Answer 7}

Appropriate citation is given in this regard.

\section{Comment 8}

Line 209. "Considering that the convection heat transfer mechanism between the receiver pipe and the glass envelope occurs by natural convection...".

\section{Answer 8}

Agreed. It was corrected. Please see the revised manuscript.

\section{Comment 9}

Line 227. The Stefan-Boltzmann constant has no measurement unit.

\section{Answer 9}

The unit is now provided. Please see the revised manuscript.

\section{Comment 10}

Line 234. "A useful heat to the working fluid...".

\section{Answer 10}

Agreed. It was corrected. Please see the revised manuscript

\section{Comment 11}

Equation (20). I think the equation has a wrong sign.

\section{Answer 11}

The equation is correct: Increase in internal energy = heat input by absorbing solar energyinternal loss-useful heat input to fluid.

\section{Comment 12}

Equation (27). I think c_f and c_p,f are the same quantity. 
Agreed. It was corrected. Please see the revised manuscript

\section{Comment 13}

Figures 3 and 11. Please provide larger figures, in the present form they are hard to analyze. Figure 11(a) reports "G" instead of "G_bt".

\section{Answer 13}

Larger figures are now given. Also, Fig. 11 (a) is corrected. Please see the revised manuscript

\section{Comment 14}

Table 2. What criterion was adopted to choose the reported PTC properties' values?

\section{Answer 14}

The used properties are reported in the literature. We added appropriate reference for them. Please see the revised manuscript

\section{Comment 15}

Line 315 and Table 3. I would say "deviation" or "error" instead of "uncertainty".

\section{Answer 15}

The word "deviation" is used as per suggestion. Please see the revised manuscript 
Energy and exergy analyses of a parabolic trough collector operated with nanofluids for medium and high temperature applications
A. Allouhi ${ }^{*}(\mathrm{a}), \mathrm{M}$.
M. Benzakour Amine ${ }^{(b)}$,
R. Saidu
T. Kousksou ${ }^{(e)}$, A. Jamil ${ }^{(a)}$

(a) Ecole Supérieure de Technologie de Fès, U.S.M.B.A, Route d'Imouzzer, BP 2427, Fez, Morocco

(b) Faculté des Sciences et Techniques de Fès, U.S.M.B.A, Route d'Imouzzer, BP 2202, Fez, Morocco

(c) Research Centre for Nano-Materials and Energy Technology (RCNMET), School of Science and Technology, Sunway University, No. 5, Jalan Universiti, Bandar Sunway, Petaling Jaya, 47500 Selangor Darul Ehsan, Malaysia

(d) Department of Engineering, Lancaster University, Lancaster, LA1 4YW, UK

(e) Laboratoire des Sciences de l'Ingénieur Appliquées à la Mécanique et au Génie Electrique (SIAME), Université de Pau et des Pays de l'Adour - IFR - A. Jules Ferry, 64000 Pau, France

\begin{abstract}
:
Thermal performance of parabolic trough collectors (PTCs) can be improved by suspending nanoparticles into the traditionally used heat transfer fluids. In this work, a one-dimensional mathematical model is proposed to investigate the effect of various nanoprticles suspended in the working fluid for medium and high temperature PTCs. The major finding of this work is that the nanofluid enhances the thermal efficiency of the PTCs slightly. High operating temperatures are more suitable for using nanofluids and generate higher relative gains of energy delivered. It is also found that the exergetic efficiency improvement is more important than energetic efficiency. The peak exergy efficiency is achieved by the $\mathrm{CuO}$ based nanofluid and is about $9.05 \%$. The maximum daily relative gain of thermal energy delivered is found to be $1.46 \%$ by using $5 \%$ of $\mathrm{Al}_{2} \mathrm{O}_{3}$ in the base fluid. Optimal control of the operating conditions can lead to optimal energetic and exergetic performances of the PTC.
\end{abstract}

Keywords: Nanofluid; PTC; heat transfer; energy efficiency; exergy efficiency 


\begin{tabular}{|c|c|c|}
\hline Symbol & Signification & Units \\
\hline $\mathrm{h}$ & Hour angle & degree \\
\hline$\delta$ & Solar declination & degree \\
\hline$\theta$ & Incidence angle & degree \\
\hline $\mathrm{k}_{\theta}$ & Incident angle modifier & dimensionless \\
\hline$\epsilon$ & Emittance & dimensionless \\
\hline $\mathrm{G}_{\mathrm{bt}}$ & Solar beam radiation & $\mathrm{W} / \mathrm{m}^{2}$ \\
\hline $\mathrm{c}$ & Specific heat capacity & $\mathrm{J} / \mathrm{kg} \mathrm{K}$ \\
\hline$h_{f}$ & $\begin{array}{l}\text { Convective heat transfer coefficient between the absorber and } \\
\text { the HTF }\end{array}$ & $\mathrm{W} / \mathrm{m}^{2} \mathrm{~K}$ \\
\hline$h_{w}$ & $\begin{array}{l}\text { Convective heat transfer coefficient between the external } \\
\text { surface of the glass cover and the ambient air }\end{array}$ & $\mathrm{W} / \mathrm{m}^{2} \mathrm{~K}$ \\
\hline$\lambda$ & Thermal conductivity & $\mathrm{W} / \mathrm{m} \mathrm{K}$ \\
\hline $\mathrm{k}_{\text {eff }}$ & $\begin{array}{l}\text { effective conductive coefficient between the glass cover and } \\
\text { absorber }\end{array}$ & $\mathrm{W} / \mathrm{m} \mathrm{K}$ \\
\hline $\mathrm{Nu}$ & Nusselt number & dimensionless \\
\hline $\operatorname{Pr}$ & Prandtl number & dimensionless \\
\hline $\mathrm{Pe}$ & Peclet number & dimensionless \\
\hline $\operatorname{Re}$ & Reynolds number & dimensionless \\
\hline $\mathrm{T}$ & temperature & $\mathrm{K}$ \\
\hline $\mathrm{v}$ & velocity & $\mathrm{m} / \mathrm{s}$ \\
\hline$\gamma$ & Intercept factor & dimensionless \\
\hline$\tau$ & transmittance & dimensionless \\
\hline$\alpha$ & absorbance coefficient & dimensionless \\
\hline $\mathrm{r}_{\mathrm{m}}$ & Reflectance of the mirror & dimensionless \\
\hline$\mu$ & DynamicViscosity & $\mathrm{kg} / \mathrm{m} \mathrm{s}$ \\
\hline$\rho$ & Density & $\mathrm{kg} / \mathrm{m}^{3}$ \\
\hline$\sigma$ & Stefan-Boltzman constant & $\mathrm{W} / \mathrm{m}^{2} \mathrm{~K}^{4}$ \\
\hline$\dot{m}$ & Fluid mass flow & $\mathrm{kg} / \mathrm{s}$ \\
\hline $\mathrm{W}_{\mathrm{a}}$ & Width of the collector & $\mathrm{m}$ \\
\hline $\mathrm{L}$ & Length of the collector & $\mathrm{m}$ \\
\hline $\mathrm{D}$ & Diameter & $\mathrm{m}$ \\
\hline A & Cross sectional area & $\mathrm{m}^{2}$ \\
\hline$\phi$ & fraction of nanoparticles & dimensionless \\
\hline$\eta$ & energetic efficiency & dimensionless \\
\hline$\eta_{\mathrm{ex}}$ & exergetic efficiency & dimensionless \\
\hline$\Delta \mathrm{e}$ & relative energy gain & dimensionless \\
\hline FoM & figure of merit & dimensionless \\
\hline
\end{tabular}




\begin{tabular}{|ll|}
\hline Subscripts & \\
$\mathrm{a}$ & Ambient \\
$\mathrm{ab}$ & Absorber \\
$\mathrm{bf}$ & Base fluid \\
$\mathrm{f}$ & Working fluid \\
$\mathrm{g}$ & Glass cover \\
$\mathrm{i}$ & Inner \\
in & Inlet \\
$\mathrm{nf}$ & Nanofluid \\
$\mathrm{np}$ & Nanoparticle \\
o & Outer \\
out & Outlet \\
$\mathrm{s}$ & Solid nanoparticle \\
Abbreviations & \\
HTF & Heat transfer fluid \\
PTC & Parabolic trough collector \\
\hline
\end{tabular}

36

37

38 


\section{Introduction}

Concerns regarding climate change are growing and the world needs to take urgent measures to avoid further warming of the earth [1]. The damaging effects of climate change are accentuated with the use of fossil fuels that are up to now considered as the main energy source for power generation worldwide [2]. As a result, increasing efforts are deployed by the research community to propose efficient and reliable alternatives for power generation mainly based on renewable energy sources [3]. Among these renewable energy resources, it is strongly believed that solar energy has the most influential potential to achieve a sustainable global energy system because of many reasons. It is clean, abundant and becoming more and more cost-effective [4]. Solar energy is one of the sustainable and potential options to fulfill a wide range of the humankind daily needs, including natural lighting [5], space and water heating [6-7], cooling [8], water desalination [9] and power generation [10]. Electrical power can be generated using photovoltaic panels by converting solar energy or solar thermal systems driven by thermodynamic cycles. The main advantages of thermal power generation over the PV one rely on the easiness of storing heat compared to electricity and the capability of thermal systems to reach higher energy productions [11]. The current available technologies used in thermal energy plants include, parabolic trough collectors [12], solar towers [13], linear Fresnel lenses [14] and dish Stirling [15]. The use of parabolic trough collectors has been successfully tested in many power generation stations worldwide due to its technological maturity and its economic competitiveness [16-18].

Recently, research related to PTCs has increased tremendously. Many researches proposed improvements in order to ameliorate the performance of PTCs. Some of them focused on proposing modifications in the absorber geometry and including objects inside the flow. Twisted tape inserts were used by Jaramillo et al. [19]. In the case of a twist ratio close to 1 and for low Reynolds numbers, their applications showed a positive effect on the performance of the collector via an enhancement of the heat transfer. Bortolato et al. [20] have studied experimentally a PTC with flat bar-and-plate absorber including an internal offset strip turbulator in the channel. The new design allowed a better efficiency (up to 64\%) with low pressure drops. Other investigators tried to test innovative working fluids such as supercritical $\mathrm{CO}_{2}$ [21] and nanofluids [22-28]. The literature review of the recently published research works has shown that there are only limited works investigating detailed analysis of PTC using nanofluids. Sokhansefat et al. [22] were the first authors to study the possibility of 
improving heat transfer in PTCs by selecting $\mathrm{Al}_{2} \mathrm{O}_{3} /$ synthetic oil nanofluid as a working fluid. A 3-D numerical model based on Navier-Stokes mass, momentum and energy equations were proposed to characterize a fully developed turbulent mixed convection heat transfer through the receiver tube. Authors reported that increasing the concentration of $\mathrm{Al}_{2} \mathrm{O}_{3}$ nanoparticles up to $5 \%$ may increase the heat transfer coefficient by $14 \%$. Ghesemi and Ranjbar [23] simulated the thermal behavior of a PTC using $\mathrm{CuO}$-water and $\mathrm{Al}_{2} \mathrm{O}_{3}$-water nanofluids. The numerical model is based on the finite volume approach and solved by a CFD commercial code. It is shown that the tested nanofluids gave better performances compared to pure water. For a volume fraction of $3 \%$, they reported an increase in the heat transfer coefficient of about $28 \%$ and $35 \%$ for $\mathrm{CuO}$-water and $\mathrm{Al}_{2} \mathrm{O}_{3}$-water nanofluids, respectively. Mwesigye et al. [24] investigated numerically the thermal and thermodynamic performance of a high concentration ratio PTC employing $\mathrm{Cu}$-Therminol VP-1 nanofluid as the working fluid. The conclusion given by the authors is that the collectors' thermal efficiency increased to $12.5 \%$ when the nanoparticle concentration varied between 0 to $6 \%$. They have also shown that by using the entropy generation method, the nanofluids can enhance thermodynamic efficiency for the certain range of Reynolds numbers. Bellos et al. [25] analyzed theoretically two options for enhancing thermal efficiency of PTCs. The first option consists of considering a dimpled receiver with a sine form. For the second option, they compared three working fluids and nanofluid was one of them. They argued that both approaches can improve the efficiency by around 4\%. An optic-thermal-stress coupling model was suggested by Wang et al. [26] in order to evaluate the influence of using $\mathrm{Al}_{2} \mathrm{O}_{3} /$ synthetic oil nanofluid as a working fluid in PTCs. The authors indicated that nanofluids enhance heat transfer, avoid high temperature gradients and minimize thermal stress receiver's deformation. Simulations were carried out by Coccia et al. [27] to analyze the energy yields of low-enthalpy parabolic trough collectors utilizing six water-based nanofluids. The authors concluded that adding low concentrations of some nanofluids lead only to minor improvements in the PTC efficiencies while high concentrations do not induce an advantage compared to water. This result originates from the fact that the dynamic viscosity increases with the weight concentration. They have therefore recommended that evaluating nanofluids (as working fluids in PTCs) at high temperatures (characterized by lower dynamic viscosities and higher thermal conductivities) could be interesting.

Based on literature survey, it was found that there are only limited investigations studying the thermal behavior of PTCs operating with nanofluids. More works with detailed analysis are 
therefore required for a good understanding of the best conditions of using nanofluids in PTC applications. Moreover, the assessment of their benefits seems to be of a particular interest, especially for medium and high temperature applications as emphasized by [27]. Another key contribution of this paper is the discussion of the effect of nanofluids on the exergetic performance of PTCs. Very limited studies were carried out on this aspect as well. In this sense, the present work presents a thermal analysis and performance assessment of PTC using three types of nanofluids as heat transfer fluids for medium and high temperature applications. The proposed mathematical model is one-dimensional and takes into account real varying external conditions in terms of incident radiation and ambient temperature for the Moroccan city "Ouarzazate". A parametric study was also conducted to show the effect of mass flow rate, inlet temperature and nanoparticle concentration on the output energy. Detailed energetic and exergetic analyses are carried out as well to identify the best conditions of nanofluid utilization in PTCs.

\section{Mathematical formalism}

\subsection{Tested fluids}

The mathematical model attempts to study heat transfer and thermal and exergetic efficiencies of a PTC using nanolfluids as working fluids. As the main focus of this paper is on medium and high-temperature heating applications, Therminol VP-1 was used as the base heat transfer fluid which is suitable for such purposes. Temperature dependent thermal properties are required for a more accurate modeling of the system. Hence, the thermal properties varying with the temperature were extracted from the manufacturer datasheet and were fitted under polynomial or exponential equations to be appropriately used by the developed code [28]. Their expressions, by considering only the liquid phase, are given below:

- Density $\left(\mathrm{kg} / \mathrm{m}^{3}\right)$ :

$$
\rho_{b f}=-2.379 \times 10^{-6} \mathrm{~T}^{3}+0.002737 \mathrm{~T}^{2}-1.871 \mathrm{~T}+1439
$$

- Specific heat capacity $(\mathrm{J} / \mathrm{kg} \mathrm{K})$

$$
c_{p_{b f}}=8.877 \times 10^{-6} \mathrm{~T}^{3}-0.01234 \mathrm{~T}^{2}+8.28 \mathrm{~T}-50.85
$$

- Thermal conductivity (W/m K)

$$
\lambda_{b f}=1.062 \times 10^{-11} \mathrm{~T}^{3}-1.937 \times 10^{-7} \mathrm{~T}^{2}+2.035 \times 10^{-5} \mathrm{~T}+0.1464
$$


- Dynamic viscosity (Pa s)

$$
\mu_{b f}=30.24 \exp (-0.03133 \mathrm{~T})+0.008808 \exp (-0.006729 \mathrm{~T})
$$

Integrating nanoparticles in the base fluid will induce an enhancement in its thermal properties. These properties are influenced by the volume fraction of the nanoparticles and their typology. Generally, this volume fraction does not exceed 5\%. The nanofluid thermal properties (i.e. density, specific heat capacity, thermal conductivity and dynamic viscosity) as a function of the volume fraction of nanoparticles $(\phi)$, are derived from the next expressions [29-31]:

$\rho_{n f}=(1-\phi) \rho_{b f}+\phi \rho_{s}$

$c_{p_{n f}} \frac{(1-\phi)\left(\rho c_{p}\right)_{b f}+\phi\left(\rho c_{p}\right)_{s}}{\rho_{n f}}$

$\lambda_{n f}=\lambda_{b f} \frac{\lambda_{s}+2 \lambda_{b f}-2 \phi\left(\lambda_{b f}-\lambda_{s}\right)}{\lambda_{s}+2 \lambda_{b f}+\phi\left(\lambda_{b f}-\lambda_{s}\right)}$

$\mu_{n f}=\mu_{b f}\left(1+2.5 \phi+6.25 \phi^{2}\right)$

In the previous equations, the subscript (nf) denotes for nanofluid, (bf) for the base fluid and (s) for the solid nanoparticles.

The study considers three oxide nanopaticle types: copper oxide $(\mathrm{CuO})$, alumina $\left(\mathrm{Al}_{2} \mathrm{O}_{3}\right)$ and titanium oxide $\left(\mathrm{TiO}_{2}\right)$. The thermal properties of these nanoparticles are given in Table 1 [3233].

\subsection{Climatic conditions}

In this work, it is suggested to study the instantaneous thermal performance of a PTC using nanofluids. A typical sunny day has been selected to run the simulation. Ambient temperature and direct beam radiations were obtained from the METEONORM database for the Moroccan city Ouarzazate. To simplify the study, an open-loop operation mode of the PTC has been considered without any coupling with a hot storage tank. This configuration has been previously proposed by Coccia et al. [27]. In the present work, a horizontal E-W axis with N- 
$\mathrm{S}$ single axis tracking is studied. The sun-tracking mechanism depends on the solar incidence angle, denoted $\theta$. The cosine of $\theta$, for a surface rotated about a horizontal east-west direction with regular adjustment is expressed as follows [34]:

$$
\cos (\theta)=\sqrt{1-\cos ^{2}(\delta) \sin ^{2}(h)}
$$

$\delta$ is the solar declination and $\mathrm{h}$ is the hour angle, all expressed in degrees.

It is interesting to note that the climatic conditions were obtained under an hourly form and were introduced into the developed code using a fifth-order polynomial interpolation.

\subsection{PTC modeling}

\subsubsection{Governing equations}

A PTC comprises a parabolic reflecting mirror that reflects the sun rays onto a receiver tube that is inserted at the focal point of the reflector. The receiver consists of a metallic absorber surrounded by a glass cover. To limit heat losses, the space between the glass cover and absorber is maintained at very low pressures. The PTC is schematically reported in Fig. 1 [35].

A one dimensional mathematical model is introduced to study the transient thermal behavior of the PTC. Therefore, the receiver tube is divided into $\mathrm{N}$ segment and heat propagation occurs according the axial direction. The inputs of the model are the instantaneous ambient temperature, incident beam radiations, mass flow rate, and physical properties of the glass cover, absorber tube and HTF.

The mathematical model is based on an energy balance in each segment of the glass envelope, absorber and the HTF. Consequently, it is imperative to compute the various heat transfer coefficients used by the model. Some simplifying hypotheses have been made:

- Incompressible HTF and unidirectional flow

- Fluid flow is uniformly distributed for each receiver segment

- Solar radiation is time dependent and is uniform around the whole receiver tube

- Conduction losses at the ends of receiver tube are neglected.

- Thermal properties of the base fluid vary with the temperature, whereas those of nanoparticles are constant.

- Thermal diffusion term in the glass cover, absorber tube and fluid are negligible 
$\dot{q}_{\text {in }}=\dot{q}_{\text {in-rad }}+\dot{q}_{\text {in-conv }}$

The radiation heat transfer mode between the receiver pipe/absorber and glass envelope can

The three coupled partial differential equations referring to the energy balances for the glass cover, absorber tube and working fluid can be expressed as follows:

\section{- Glass cover:}

The glass cover receives solar radiation along its outer surface, exchanges heat with both the absorber tube and the ambient. Energy balance for the glass cover is given as:

$$
A_{g} \rho_{g} c_{g} \frac{\partial T_{g}}{\partial t}=\dot{q}_{s-g}(t)+\dot{q}_{\text {in }}(x, t)-\dot{q}_{\text {out }}(x, t)
$$

The solar radiation received by the glass cover $\dot{q}_{s-g}(t)$ can be considered as a heat flux. This can be justified by the fact that the glass cover is significantly thin and possesses a very low absorptance coefficient of the order of 0.02. It can be expressed as:

$\dot{q}_{s-g}(t)=\gamma \alpha_{g} r_{m} W_{a} G_{b t}(t) k_{\theta}(t)$

This term depends on the available instantaneous beam solar radiation $\left(\mathrm{G}_{\mathrm{bt}}\right)$, collector width $\left(\mathrm{W}_{\mathrm{a}}\right)$ and other optical properties including intercept factor $(\gamma)$, absorbance of glass cover $\left(\alpha_{\mathrm{g}}\right)$, specular reflectance of the mirror $\left(\mathrm{r}_{\mathrm{m}}\right)$ and the incident angle modifier $\left(\mathrm{k}_{\theta}\right)$. The incident angle modifier is given as a fourth-order polynomial form of the incident angle [36]:

$k_{\theta}=1-2.2307 \times 10^{-4} \theta-1.1 \times 10^{-4} \theta^{2}+3.18596 \times 10^{-6} \theta^{3}-4.85509 \times 10^{-8} \theta^{4}$

All the parameters of Eq. (11) together with other geometrical properties of the PTC are specified in Table 2 [37].

Internal heat transfer between the absorber and the glass envelope heat transfer occur by convection and radiation, thus: be written as: 
$212 \dot{q}_{i n-r a d}=\frac{\pi D_{o-g}\left(T_{a b}^{4}-T_{g}^{4}\right)}{\frac{1}{\epsilon_{a b}}+\frac{1-\epsilon_{g}}{\epsilon_{g}} \frac{D_{a b-o}}{D_{g-i}}}$

213 Considering that the convection heat transfer mechanism between the receiver pipe and glass

214 envelope occurs by natural convection due to the presence of a pressure $>0.013 \mathrm{~Pa}$, one can

215 use the Raithby and Holland's formula to characterize the convection heat transfer between

216 the absorber tube and glass cover wall [38]

217

$$
\dot{q}_{i n-c o n v}=\frac{2 \pi k_{e f f}\left(T_{a b}-T_{g}\right)}{\ln \left(\frac{D_{g-i}}{D_{a b-o}}\right)}
$$

218 Heat exchange between the glass cover and the atmosphere takes place by convection and

219 radiation. Due to the presence of wind, the Newton's law of cooling can be employed to 220 determine the convective heat loss as [34]:

$\dot{q}_{\text {out-conv }}=\pi D_{g-o} h_{w}\left(T_{g}-T_{a}\right)$

222 with:

223

$h_{w}=\frac{N u_{a i r} k_{a i r}}{D_{g-o}}$

224 and

225

$N u_{\text {air }}=\left\{\begin{array}{l}0.4+0.54 \operatorname{Re}_{\text {air }}{ }^{0.52} \text { if } 0.1<\operatorname{Re}_{\text {air }}<1000 \\ 0.3 \operatorname{Re}_{\text {air }}{ }^{0.6} \text { if } 1000<\operatorname{Re}_{\text {air }}<50000\end{array}\right.$

226 Taking the assumption that the cover is a small convex gray object in a large black body

227 cavity, the sky, one can estimate the radiation heat exchange by:

$\dot{q}_{\text {out }-\mathrm{rad}}=\pi D_{g-o} \in_{g} \sigma\left(T_{g}^{4}-T_{\text {sky }}^{4}\right)$

229 In the previous equations $\mathrm{T}_{\mathrm{g}}, \mathrm{T}_{\mathrm{a}}$ and $\mathrm{T}_{\text {sky }}$ correspond to the outer glass cover temperature, ambient temperature, respectively. $\mathrm{T}_{\text {sky }}$ is the sky temperature taken as $T_{s k y}=0.0552 T_{a}^{1.5}$ 
$\sigma$ is the Stefan-Boltzman constant $\left(\sigma=5.67 \times 10^{-8} \mathrm{~W} / \mathrm{m}^{2} \mathrm{~K}^{4}\right)$ while $\epsilon_{\mathrm{g}}$ and $\epsilon_{\mathrm{ab}}$ are the emittance of the glass cover and absorber, respectively. $k_{\text {eff }}$ is the effective conductive coefficient between the glass cover and absorber, and D denotes the diameter with subscripts ab-o for

$$
A_{a b} \rho_{a b} c_{a b} \frac{\partial T_{a b}}{\partial t}=\dot{q}_{s-a b}(t)-\dot{q}_{i n}(x, t)-\dot{q}_{u}(x, t)
$$

241 The term $\dot{q}_{s-a b}(t)$ refers to the solar energy absorbed by the PTC receiver. It can be put under 242 the following form:

$$
\dot{q}_{s-a b}(t)=\gamma\left(\tau_{g} \alpha_{a b}\right) r_{m} W_{a} k_{\theta}(t) G_{b t}(t)
$$

or:

$$
\dot{q}_{s-a b}(t)=\dot{q}_{s-g}(t) \frac{\left(\tau_{g} \alpha_{a b}\right)}{\alpha_{g}}
$$

$\dot{q}_{u}(x, t)=\pi D_{a b-i} h_{f}\left(T_{a b}-T_{f}\right)$

$252 D_{a b-i}$ is the inner diameter of the absorber and $T_{f}$ is the HTF temperature. $h_{f}$ is the convection 
254

255

256

257

258

259

thermal properties of the working fluid. This coefficient is determined based on the Nusselt number value. Here, two correlations are used referring to the case of the base fluid and to the case of nanofluids. The first correlation, depending on Reynolds and Prandtl numbers, called the Dittus-boelter correlation estimates the Nusselt number as follows [39]:

$N u_{b f}=0.023 \operatorname{Re}_{b f}{ }^{0.8} \operatorname{Pr}_{b f}{ }^{0.4}$

In the case of nanofluid, Xuan et al. [40] proposed the following formulation to estimate the Nusselt number:

$N u_{n f}=0.0059\left(1.0+7.628 \phi^{0.6886} P e_{n p}^{0.001}\right) \operatorname{Re}_{n f}{ }^{0.9238} \operatorname{Pr}_{n f}{ }^{0.4}$

where $\mathrm{Pe}_{\mathrm{np}}$ is the Peclet number describing the effect of thermal dispersion because of microconvective and microdiffusion of the suspended nanoparticles. It is given as:

$P e_{n p}=\frac{v_{n f} \times d_{n p}}{\alpha_{n f}}$

with $\mathrm{v}_{\mathrm{nf}}$ is the nanofluid velocity, $\mathrm{d}_{\mathrm{np}}$ is the nanoparticle diameter and $\alpha_{\mathrm{nf}}$ is the thermal diffusivity of nanofluid. Reynolds and Prandtl numbers are evaluated by considering the temperature-dependent thermal properties of each nanofluid type.

It is also interesting to highlight that the two previous correlations are recommended in the case of turbulent flows. In this sense, simulation tests were carried out to determine the mass flow range with respect to this condition.

\section{- Working fluid}

The working fluid flows inside the absorber at a flow rate $\dot{m}$ and absorbs heat by convection from the inner absorber tube. The energy balance of the HTF can take the following form:

$$
A_{f} \rho_{f} c_{f} \frac{\partial T_{f}}{\partial t}+\dot{m} c_{f} \frac{\partial T_{f}}{\partial x}-k_{f} A_{f} \frac{\partial^{2} T_{f}}{\partial x^{2}}=\dot{q}_{u}(x, t)
$$

In all the governing equations $\mathrm{A}, \rho$ and $\mathrm{c}$ denotes for the cross-sectional area $\left(\mathrm{m}^{2}\right)$, density $\left(\mathrm{kg} / \mathrm{m}^{3}\right)$ and specific heat capacity $(\mathrm{J} / \mathrm{kg} \mathrm{K})$. Also, it is noteworthy to mention that all the equations are referred to the length unit of the collector. 
The initial conditions of the energy balance equations were introduced by considering that at 280 time $\mathrm{t}=0$, the glass cover, absorber tube and HTF are all in thermal equilibrium with the atmosphere. Moreover, the boundary conditions were implemented considering that at $\mathrm{x}=0$, the temperatures are constant and refer to the inlet fluid temperature.

\subsection{Performance indices}

The present work suggests assessing the performance of the solar PTC by comparing the outlet temperature of the working fluid (that can be base fluid or one of tested nanofluids), the energetic efficiency the PTC, its exergetic efficiency and the relative benefit of the useful energy delivered for the various working fluids.

The impact on these indices is the result of the improvement of the heat coefficient transfer $h_{f}$. The Figure of Merit (FoM) expressing the ratio of the heat transfer coefficient (nanofluid cases and base fluid case) is a useful criterion to judge the benefit of nanofluids versus the base fluid. It is given as [41]:

291

$$
F o M=\frac{\left.h_{6}\right|_{2, f}}{\left.h_{f}\right|_{b f}}
$$

The outlet temperature of the HTF is determined by solving the previous set of equations and corresponds to:

$T_{\text {out }}=T_{f}(x=L)$

The instantaneous energetic efficiency refers to the ratio between the useful thermal energy gained by the working fluid to the available solar beam energy falling onto the PTC reflector. It is expressed as:

$299 \quad \eta=\frac{\dot{Q}_{u}}{A_{a} G_{b t}}=\frac{\dot{m} \int_{T_{i n}}^{T_{\text {out }}} c_{f}(T) d T}{W_{a} L G_{b t}}$ 
The exergetic efficiency can be defined as the ratio of gain exergy $\left(\mathrm{E}_{\mathrm{u}}\right)$ to available solar radiation exergy $\left(\mathrm{E}_{\mathrm{s}}\right)$ and can be expressed as [42]:

302

$$
\eta_{\text {ex }}=\frac{E_{u}}{E_{s}}=\frac{\dot{m} \int_{T_{\text {in }}}^{T_{\text {out }}} c_{f}(T) d T-T_{a} \int_{T_{\text {in }}}^{T_{\text {out }}} \frac{c_{f}(T)}{T} d T}{W_{a} L G_{b t}\left[1-\frac{4}{3}\left(\frac{T_{a}}{T_{\text {sun }}}\right)+\frac{1}{3}\left(\frac{T_{a}}{T_{\text {sun }}}\right)^{4}\right]}
$$

303

In Eq. (31), $\mathrm{T}_{\text {sun }}$ is the sun's apparent temperature taken to be $6000 \mathrm{~K}$ as mentioned by Petela [43].

The last performance indicator is the relative energy gain resulting from the difference between the energy delivered by the PTC when the nanofluids are used compared to the base fluid. It is given as

$\Delta e=\frac{Q_{u-n f}-Q_{u-b f}}{Q_{u-b f}} \times 100$

The flow diagram, showing the inputs, the outputs and the calculations operated by the model is presented in Fig. 2.

Proving the validity of the proposed mathematical model is essential before further exploitation of its results. Therefore, a validation was performed based on a comparison between our model and experimental tests of Sandia National Laboratory (SNL) [44]. The SNL has experimentally tested a small module of LS-2 collector at the AZTRAK rotating platform to analyze the effect of various conditions on the PTC performance which can help in minimizing operation and maintenance costs of CSP plants. The code of the present model has been run in similar conditions as in [44] considering the same working fluid (Syltherm 800 oil) and the same geometrical properties of the PTC. Three test conditions were considered for the validation that is based on the outlet temperature and the thermal efficiency. The results are given in Table 3. It is clear that the results of the model in terms of outlet temperature and thermal efficiency are in very good agreement with the measured data 
(uncertainty $<0.83{ }^{\circ} \mathrm{C}$ for the temperature and $<2.9 \%$ for the efficiency). This proves that the developed mathematical model is valid.

\section{Results and discussion}

Several MATLAB subroutines were built to compute various inputs for the main program. The main program includes the discretization of the differential equations and resolution of the obtained algebraic equations. At each time iteration, the non-linear aspect of the problem is handled by considering the temperature-dependent thermal properties at the previous time step. When the temperature of the glass cover, absorber and HTF are known, the program computes the performance indices on a time-evolution basis.

Climatic input data were load from MS Excel data after a pre-processing of the cosine of incident angle accounting for the sun-tracking strategy (i.e. N-S tracking). As stated before, a typical sunny day in the region of Ouarzazate (Morocco) is considered. The climatic data are depicted in Fig. 3. A maximum ambient temperature of $308 \mathrm{~K}$ is recorded at $15 \mathrm{~h} 00$ am while the minimum one is recorded at the sunrise (291 K). Fig. 3 also shows the hourly variation of the incident beam radiation between the sunrise and the sunset. The peak solar radiation is observed at midday and is about $1000 \mathrm{~W} / \mathrm{m}^{2}$. Other subroutines were developed in order to compute the term sources of the governing equations. The various properties of the tested fluids with respect to the temperature are used at each time step for a more accurate resolution. The generated data are used by the main program and serve in determining the heat transfer coefficient and other involved parameters figuring in the governing equations. Fig. 4 plots thermal properties of the base fluid together with the tested nanofluids for temperatures ranging from $300 \mathrm{~K}$ to $650 \mathrm{~K}$. It is clear that nanofluids possess higher densities than the base fluid (see Fig. 4 (a)). All fluids have a descending behavior of density with increasing temperatures. Increasing the concentration of nanoparticles induces further increase in the density. Also, it is clear that $\mathrm{Cu}-\mathrm{O}$ nanoparticles have a more pronounced effect on the increase of the density if compared to other types. Obviously, the presence of nanofluids leads to an enhancement of the thermal conductivity of HTF, as indicated in Fig. 4 (b). It is shown that $\mathrm{TiO}_{2}$ based nanofluid has a slightly lower thermal conductivity compared to the other nanofluids that have approximately the same values. This is surely because $\mathrm{TiO}_{2}$ nanoparticles have lower thermal conductivity (see Table 1). Moreover, by increasing the concentration of nanoparticles, thermal conductivities increase as well. By increasing the temperature, one can see that the relative gain in terms of the enhancement of the thermal 
conductivity is reduced independently of the nature of nanoparticles. The specific heat capacity, as indicated in Fig. 4 (c), gets decreased by using nanofluids. The most influential effect is shown for the case of $\mathrm{CuO}$ based nanofluid. The two other nanofluids have approximately at low concentration of nanoparticles, but as the concentration of nanoparticles increases, the difference between their specific heat capacities becomes greater.

Fig. 4 (d) shows the variation of dynamic viscosity versus the temperature. The main observation is that, at higher temperatures, adding nanoparticles to the base fluid, have a negligible effect on the viscosity. Also, as the nanoparticle concentration increases, the working fluid becomes more viscous. Such tendency is clearer at low temperatures. The changes on the thermal properties of the working fluids will certainly affect its thermal performance.

Based on these thermal properties, it was possible to generate plots of the convective heat transfer coefficient. Besides, the two correlations of the Nusselt number (Eq. (24) and Eq. (25)) referring to the base fluid case and the nanofluid case were used in the computational procedure. Fig. 5 shows the trend of this coefficient for various operating conditions, considering the case of the base fluid. It is seen that the heat convection coefficient increases with increasing temperatures (from $120 \mathrm{~W} / \mathrm{m}^{2} \mathrm{~K}$ at $300 \mathrm{~K}$ to $420 \mathrm{~W} / \mathrm{m}^{2} \mathrm{~K}$ at $650 \mathrm{~K}$ ). The curve slope is a little more important for temperatures $<400 \mathrm{~K}$.

For the sake of comparison, a 3-D representation showing the variation of the convective heat transfer coefficient in the case of the $\mathrm{CuO}$ based nanofluid is illustrated in Fig. 6. It can be clearly seen that the presence of $\mathrm{CuO}$ nanoparticles considerably enhances the convective heat transfer coefficient. This enhancement is of the order of $32 \%-83 \%$ at a maximum operating temperature of $650 \mathrm{~K}$, when compared to the base fluid. Lower operating temperatures lead to lower improvements. This makes sense to the hypothesis of the suitability of nanofluids for PTC applications involving high temperatures. This result is supported by the behavior of the Figure of Merit (FoM) illustrated in Fig. 7. It is clear that in general the FoM is greater than 1 (except at very low concentrations at low operating temperatures). A maximum FoM of 1.9 is reached at a temperature of $650 \mathrm{~K}$ and at a concentration of nanoparticles equal to $5 \%$.

Simulations were carried out to evidence the effect of using nanofluids in PTCs instead of the base fluid. The resolution of the governing equations has permitted to predict the temporary thermal behavior of the PTC. Considering the base fluid, a mass flow rate of $0.5 \mathrm{~kg} / \mathrm{s}$ and an inlet temperature of $323 \mathrm{~K}\left(50{ }^{\circ} \mathrm{C}\right)$, Fig. 8 shows the instantaneous variation of the fluid 
temperature along the day and along the axial direction of the PTC. As the working fluid flows inside the absorber, it gets gradually heated. The maximum temperature is reached at the outlet of the collector when the incident beam radiation is at its peak value (midday).

The next set of results illustrates the effect of using nanofluids as working fluids in the PTC. The same previous operating conditions were considered. The temporary evolution of the outlet temperature is depicted in Fig. 9. The nanoparticle concentration was set to a value of $\phi=3 \%$. One can see clearly that the nanofluids reach higher temperatures than the base fluid, especially at high radiation levels inducing greater heat propagation in the absorber and working fluid. $\mathrm{CuO}$ based nanofluid leads to the most significant increase in the outlet temperature while the other nanofluids give approximately the same thermal response with a little advantage of $\mathrm{TiO}_{2}$ based nanofluid. Based on this, the calculation of thermal efficiency and exergy efficiency was numerically investigated by evaluating the integrals expressions in Eqs. (30)-(31) using the trapezoidal method. The results are reported in Fig. 10 and Fig. 11, respectively.

Fig. 10 shows a minor improvement of the thermal efficiency of the PTC when nanofluids are used instead of the base fluid with no significant difference between the tested nanofluids. It is because the inlet temperature is fixed to $323 \mathrm{~K}$ which does not allow considerable improvements of the convective heat transfer coefficient $h_{f}$ as highlighted in Figs. 5 and 6. The enhancement of the exergy efficiency is more significant than the thermal efficiency (see Fig. 11). This result can be justified by the fact that the specific heat capacity of the nanofluid is considerably less important than the one of the base fluid which induces a more pronounced increase on the exergy output $\mathrm{E}_{\mathrm{u}}$ (see Eq. (31) and Fig. 3 (c)).

Fig. 12 shows the thermal efficiency and exergy efficiency plotted against the parameter $\left(T_{i n}-T_{a}\right) / G_{b t}$ supposing a constant inlet temperature of $323 \mathrm{~K}$ and a mass flow rate of $0.5 \mathrm{~kg} / \mathrm{s}$. It is shown that both thermal and exergy efficiencies follow a decreasing trend with respect to the defined ratio, with a sharper decrease for the thermal efficiency. For the base fluid, the maximum thermal efficiency is found to reach $65.7 \%$, while the minimum is about $43 \%$ with only a marginal benefit when using nanofluids. The exergy efficiency ranges between $3.05 \%$ and $8.5 \%$ for the base fluid case and gets improved more remarkably when nanofluids are employed. The peak exergy efficiency is attained by the $\mathrm{CuO}$ based nanofluid and is about $9.05 \%$. 
In order to evidence the combined effect of mass flow rate and inlet temperature, a parametric study was carried out comparing the energy and exergy efficiencies of the base fluid and $\mathrm{CuO}$ based nanofluid (as an example) for various conditions. This was made considering climatic conditions referring to the maximum solar radiation (observed at midday).

The results are plotted in Fig. 13 and Fig. 14. It is shown that, for the selected conditions, the thermal efficiency of the PTC follows a decreasing tendency with increasing inlet temperature independently of the working fluid nature. Increasing the mass flow rate generates a slight increase in the thermal efficiency. This increase is less important when the mass flow rate becomes higher. Comparing Fig. 13 (a) and Fig. 13 (b), one can remark that the presence of $\mathrm{CuO}$ nanoparticles in the base fluid enhances slightly the thermal efficiency, especially at higher temperatures.

From Fig. 14, it can be seen that the exergy efficiency increases as the inlet temperature increases, which is the opposite tendency for the thermal efficiency. Also, the mass flow rate impacts a little the exergy efficiency. The difference between the exergy efficiencies (base fluid and nanofluid) is also observed to be more important at increased inlet temperatures.

Relative daily energy gains associated with the use of nanofluids instead of the base fluid for various operating conditions in terms of mass flow rate, inlet temperature, nanoparticle type and concentration are given in Tables 4-5.

In Table 4, it is considered that the inlet temperature is set to a value of $323 \mathrm{~K}\left(50{ }^{\circ} \mathrm{C}\right)$. The observations that can be made are: (i) low concentrations of nanoparticles induce only minor improvements on the relative daily energy gains at high flow rates and are not advised at all for low flow rates; (ii) The nanoparticle type has a small effect of the gains with a certain advantage of $\mathrm{Al}_{2} \mathrm{O}_{3}$ nanoparticles; (iii) Increasing the mass flow rate has a minor positive effect of the relative daily energy gain.

Table 5 shows that increasing the inlet temperature generates a more considerable improvement of the relative daily energy gain. This is mainly due to the improvement occurring in the heat transfer coefficient at higher operating temperatures. From these two tables one can conclude that the best combination of mass flow rate and inlet temperature is when both are maximized. The maximum daily relative gain that can be reached is about 1.46 $\%$ by using $5 \%$ of $\mathrm{Al}_{2} \mathrm{O}_{3}$ in the base fluid. 
Another global conclusion that can be drawn is that operating conditions affect differently the energy and exergy related indicators, especially in terms of inlet temperature. Further detailed optimization should be conducted to ensure the best combination of design parameters selection based on the solar application.

\section{Conclusion}

A validated and detailed mathematical model was proposed to examine the benefits of using nanofluids as working fluids in parabolic trough collectors for medium and high temperature applications. Energy and exergy analyses were carried out based on real fluctuating operating conditions. Nanoparticles type and concentration, mass flow rate and inlet temperature were the parameters studied and the performance indices included the Figure of Merit, instantaneous outlet leaving the collector, thermal efficiency, exergy efficiency and relative gain in the thermal energy delivered to the utilization. The following conclusions have been made:

- Presence of nanoparticles in the base fluid enhances the convective heat transfer and can lead to higher values of the FoM. For Cuo based nanofluid, the FoM is greater than 1 for nanoparticle concentration $>1 \%$ and can exceed 1.8 at an operating temperature of $650 \mathrm{~K}$ and a nanoparticle concentration of $5 \%$.

- Nanofluids achieved higher temperatures than the base fluid, especially at higher levels of radiation. $\mathrm{CuO}$ based nanofluid leads to the most significant increase in the outlet temperature while the other nanofluids give approximately the same thermal behavior with a small advantage of $\mathrm{TiO}_{2}$ based nanofluid

- For a nanoparticle concentration of $3 \%$, only a minor improvement of the thermal efficiency of the PTC when nanofluids are used instead of the base fluid with no significant difference between the tested nanofluids.

- For similar conditions, the enhancement of the exergy efficiency is more significant than the thermal efficiency.

- The exergy efficiency varied between $3.05 \%$ and $8.5 \%$ for the base fluid case and gets improved more remarkably when nanofluids are employed. The peak exergy efficiency is attained by the $\mathrm{CuO}$ based nanofluid and is about $9.05 \%$.

- The maximum daily relative gain in terms of thermal energy delivered that is about $1.46 \%$ by using $5 \%$ of $\mathrm{Al}_{2} \mathrm{O}_{3}$ in the base fluid. 
- The parametric analysis showed that the operating conditions (i.e. mass flow rate and inlet temperature) should be carefully controlled for optimal energetic and exergetic performances. 


\section{References}

1. Pachauri, R. K., Allen, M. R., Barros, V. R., Broome, J., Cramer, W., Christ, R., ... \& Dubash, N. K. (2014). Climate change 2014: synthesis report. Contribution of Working Groups I, II and III to the fifth assessment report of the Intergovernmental Panel on Climate Change (p. 151). IPCC.

2. Liddle, B., \& Sadorsky, P. (2017). How much does increasing non-fossil fuels in electricity generation reduce carbon dioxide emissions?. Applied Energy, 197, 212221.

3. Wüstenhagen, R., \& Menichetti, E. (2012). Strategic choices for renewable energy investment: Conceptual framework and opportunities for further research. Energy Policy, 40, 1-10.

4. Solangi, K. H., Islam, M. R., Saidur, R., Rahim, N. A., \& Fayaz, H. (2011). A review on global solar energy policy. Renewable and sustainable energy reviews, 15(4), 2149-2163.

5. Michael, A., \& Heracleous, C. (2017). Assessment of natural lighting performance and visual comfort of educational architecture in Southern Europe: The case of typical educational school premises in Cyprus. Energy and Buildings, 140, 443-457.

6. Streicher, W. (2015). Solar thermal technologies for domestic hot water preparation and space heating. Renewable Heating and Cooling: Technologies and Applications, 9.

7. Allouhi, A., Jamil, A., Kousksou, T., El Rhafiki, T., Mourad, Y., \& Zeraouli, Y. (2015). Solar domestic heating water systems in Morocco: an energy analysis. Energy Conversion and Management, 92, 105-113.

8. Allouhi, A., Kousksou, T., Jamil, A., Bruel, P., Mourad, Y., \& Zeraouli, Y. (2015). Solar driven cooling systems: An updated review. Renewable and Sustainable Energy Reviews, 44, 159-181.

9. Kabeel, A. E., \& El-Agouz, S. A. (2011). Review of researches and developments on solar stills. Desalination, 276(1), 1-12.

10. Hogerwaard, J., Dincer, I., \& Naterer, G. F. (2017). Solar energy based integrated system for power generation, refrigeration and desalination. Applied Thermal Engineering, 121, 1059-1069.

11. Khan, J., \& Arsalan, M. H. (2016). Solar power technologies for sustainable electricity generation-A review. Renewable and Sustainable Energy Reviews, 55, 414-425. 
12. Fernández-García, A., Zarza, E., Valenzuela, L., \& Pérez, M. (2010). Parabolic-trough solar collectors and their applications. Renewable and Sustainable Energy Reviews, 14(7), 1695-1721.

13. Flueckiger, S. M., Iverson, B. D., Garimella, S. V., \& Pacheco, J. E. (2014). Systemlevel simulation of a solar power tower plant with thermocline thermal energy storage. Applied Energy, 113, 86-96.

14. Perini, S., Tonnellier, X., King, P., \& Sansom, C. (2017). Theoretical and experimental analysis of an innovative dual-axis tracking linear Fresnel lenses concentrated solar thermal collector. Solar Energy, 153, 679-690.

15. Ahmadi, M. H., Ahmadi, M. A., Mellit, A., Pourfayaz, F., \& Feidt, M. (2016). Thermodynamic analysis and multi objective optimization of performance of solar dish Stirling engine by the centrality of entransy and entropy generation. International Journal of Electrical Power \& Energy Systems, 78, 88-95.

16. Boukelia, T., \& Mecibah, M. S. (2013). Parabolic trough solar thermal power plant: Potential, and projects development in Algeria. Renewable and Sustainable Energy Reviews, 21, 288-297.

17. Kaygusuz, K. (2011). Prospect of concentrating solar power in Turkey: the sustainable future. Renewable and Sustainable Energy Reviews, 15(1), 808-814.

18. Kousksou, T., Allouhi, A., Belattar, M., Jamil, A., El Rhafiki, T., Arid, A., \& Zeraouli, Y. (2015). Renewable energy potential and national policy directions for sustainable development in Morocco. Renewable and Sustainable Energy Reviews, 47, 46-57.

19. Jaramillo, O. A., Borunda, M., Velazquez-Lucho, K. M., \& Robles, M. (2016). Parabolic trough solar collector for low enthalpy processes: An analysis of the efficiency enhancement by using twisted tape inserts. Renewable Energy, 93, 125-141.

20. Bortolato, M., Dugaria, S., \& Del Col, D. (2016). Experimental study of a parabolic trough solar collector with flat bar-and-plate absorber during direct steam generation. Energy, 116, 1039-1050.

21. Qiu, Y., Li, M. J., He, Y. L., \& Tao, W. Q. (2016). Thermal performance analysis of a parabolic trough solar collector using supercritical $\mathrm{CO} 2$ as heat transfer fluid under non-uniform solar flux. Applied Thermal Engineering, 115, 1255-1265

22. Sokhansefat, T., Kasaeian, A. B., \& Kowsary, F. (2014). Heat transfer enhancement in parabolic trough collector tube using Al2O3/synthetic voil nanofluid. Renewable and Sustainable Energy Reviews, 33, 636-644. 
23. Ghasemi, S. E., \& Ranjbar, A. A. (2016). Thermal performance analysis of solar parabolic trough collector using nanofluid as working fluid: a CFD modelling study. Journal of Molecular Liquids, 222, 159-166.

24. Mwesigye, A., Huan, Z., \& Meyer, J. P. (2016). Thermal performance and entropy generation analysis of a high concentration ratio parabolic trough solar collector with Cu-Therminol@ VP-1 nanofluid. Energy Conversion and Management, 120, 449-465.

25. Bellos, E., Tzivanidis, C., Antonopoulos, K. A., \& Gkinis, G. (2016). Thermal enhancement of solar parabolic trough collectors by using nanofluids and convergingdiverging absorber tube. Renewable Energy, 94, 213-222.

26. Wang, Y., Xu, J., Liu, Q., Chen, Y., \& Liu, H. (2016). Performance analysis of a parabolic trough solar collector using A12O3/synthetic oil nanofluid. Applied Thermal Engineering, 107, 469-478.

27. Coccia, G., Di Nicola, G., Colla, L., Fedele, L., \& Scattolini, M. (2016). Adoption of nanofluids in low-enthalpy parabolic trough solar collectors: Numerical simulation of the yearly yield. Energy Conversion and Management, 118, 306-319.

28. https://www.therminol.com/products/Therminol-VP1

29. Xuan, Y., \& Roetzel, W. (2000). Conceptions for heat transfer correlation of nanofluids. International Journal of heat and Mass transfer, 43(19), 3701-3707.

30. Shahrul, I. M., Mahbubul, I. M., Khaleduzzaman, S. S., Saidur, R., \& Sabri, M. F. M. (2014). A comparative review on the specific heat of nanofluids for energy perspective. Renewable and Sustainable Energy Reviews, 38, 88-98.

31. Solangi, K. H., Kazi, S. N., Luhur, M. R., Badarudin, A., Amiri, A., Sadri, R., ... \& Teng, K. H. (2015). A comprehensive review of thermo-physical properties and convective heat transfer to nanofluids. Energy, 89, 1065-1086.

32. Kamyar, A., Saidur, R., \& Hasanuzzaman, M. (2012). Application of computational fluid dynamics (CFD) for nanofluids. International Journal of Heat and Mass Transfer, 55(15), 4104-4115.

33. Faizal, M., Saidur, R., Mekhilef, S., \& Alim, M. A. (2013). Energy, economic and environmental analysis of metal oxides nanofluid for flat-plate solar collector. Energy Conversion and Management, 76, 162-168.

34. Kalogirou, S. A. (2013). Solar energy engineering: processes and systems. Academic Press. 
35. Allouhi, A., Amine, M. B., Kousksou, T., Jamil, A., \& Lahrech, K. (2018). Yearly performance of low-enthalpy parabolic trough collectors in MENA region according to different sun-tracking strategies. Applied Thermal Engineering, 128, 1404-1419

36. Mokheimer, E. M., Dabwan, Y. N., Habib, M. A., Said, S. A., \& Al-Sulaiman, F. A. (2014). Techno-economic performance analysis of parabolic trough collector in Dhahran, Saudi Arabia. Energy Conversion and Management, 86, 622-633.

37. Shahin, M. S., Orhan, M. F., \& Uygul, F. (2016). Thermodynamic analysis of parabolic trough and heliostat field solar collectors integrated with a Rankine cycle for cogeneration of electricity and heat. Solar Energy, 136, 183-196.

38. Kalogirou, S. A. (2012). A detailed thermal model of a parabolic trough collector receiver. Energy, 48(1), 298-306.

39. Erdogan, A., Colpan, C. O., \& Cakici, D. M. (2017). Thermal design and analysis of a shell and tube heat exchanger integrating a geothermal based organic Rankine cycle and parabolic trough solar collectors. Renewable Energy, 109, 372-391.

40. Li, Q., Xuan, Y., \& Wang, J. (2003). Investigation on convective heat transfer and flow features of nanofluids. Journal of Heat transfer, 125(2003), 151-155.

41. Gómez-Villarejo, R., Martín, E. I., Navas, J., Sánchez-Coronilla, A., Aguilar, T., Gallardo et al. (2017). Ag-based nanofluidic system to enhance heat transfer fluids for concentrating solar power: Nano-level insights. Applied Energy, 194, 19-29.

42. Padilla, R. V., Fontalvo, A., Demirkaya, G., Martinez, A., \& Quiroga, A. G. (2014). Exergy analysis of parabolic trough solar receiver. Applied Thermal Engineering, 67(1), 579-586.

43. Petela, R. (2003). Exergy of undiluted thermal radiation. Solar Energy, 74(6), 469488.

44. Dudley, V., Kolb, G., Sloan, M., \& Kearney, D. (1994). SEGS LS2 solar collectorTest results. Report of Sandia National Laboratories, Report No. SANDIA94-1884. 


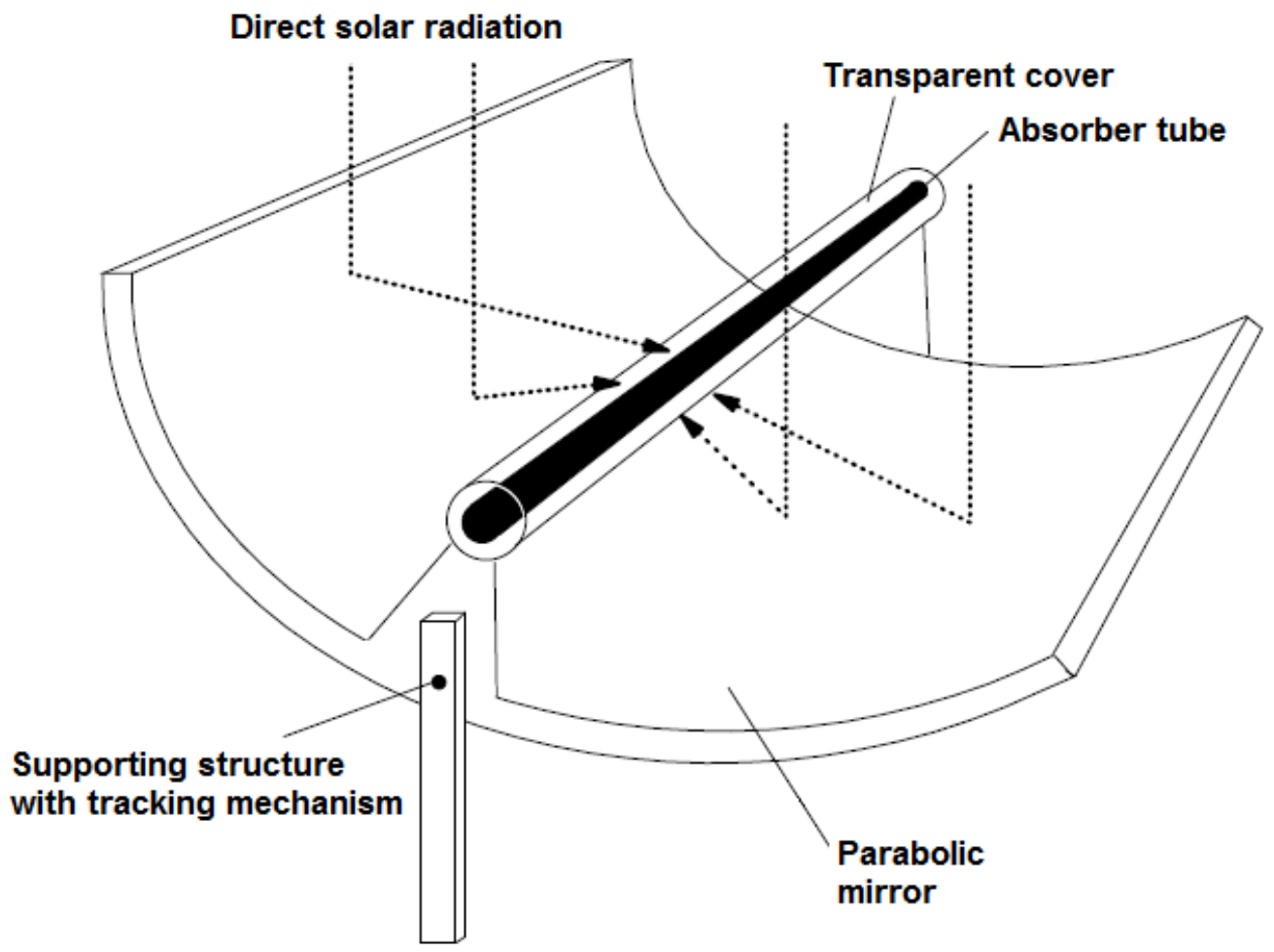




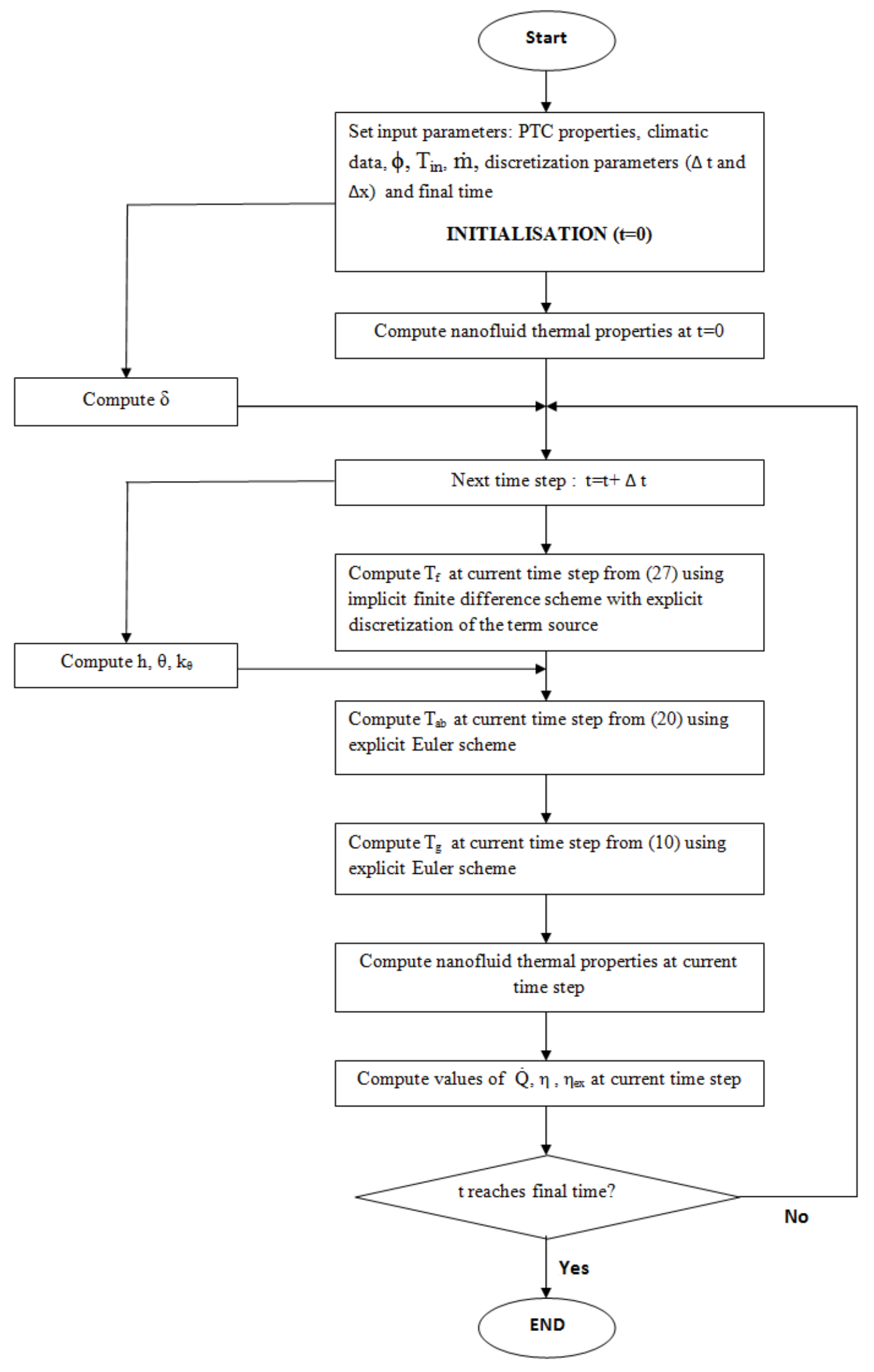




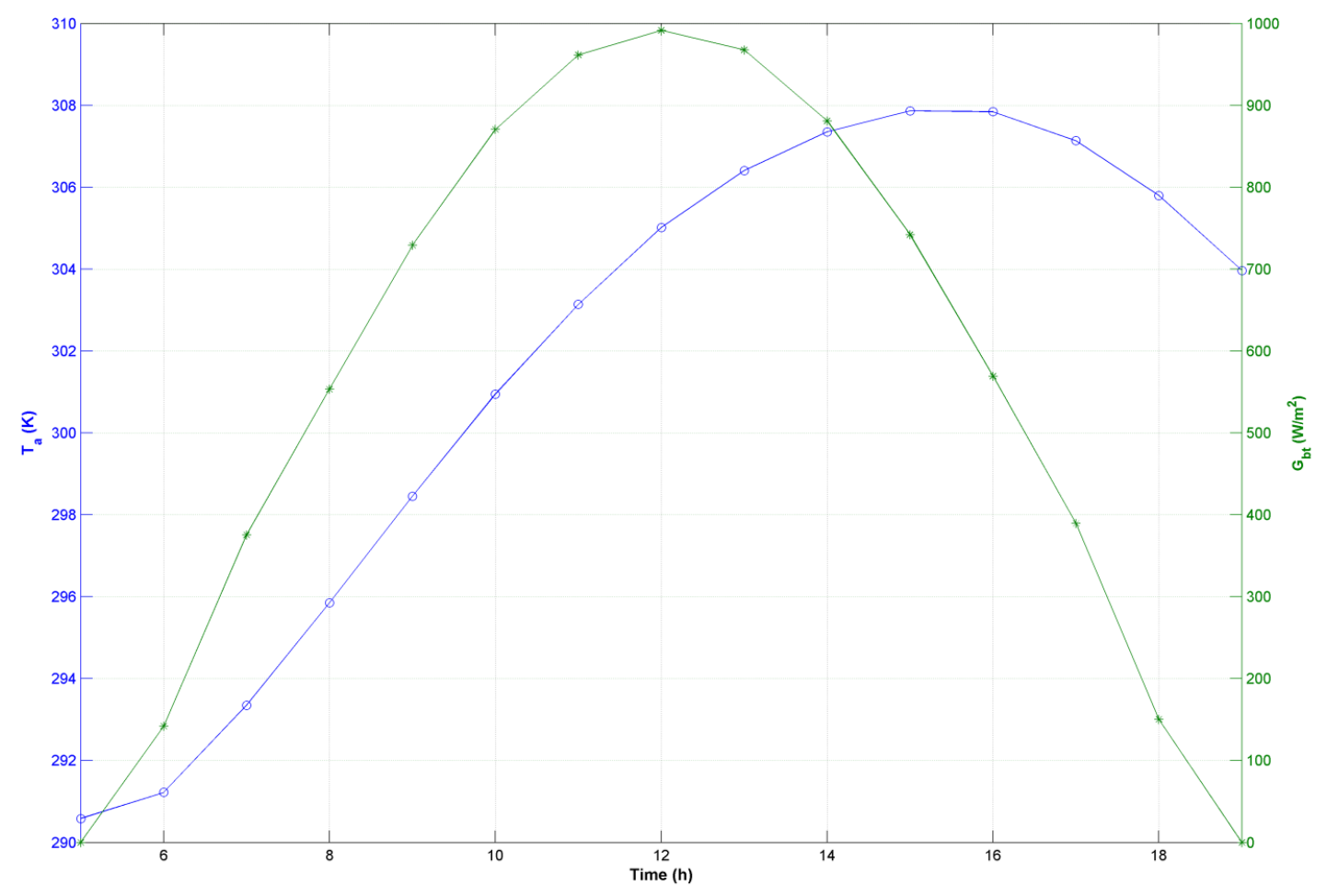

Fig. 3: Hourly variation of the ambient temperature (left axis) and beam incident 618 radiation (right axis)

619 


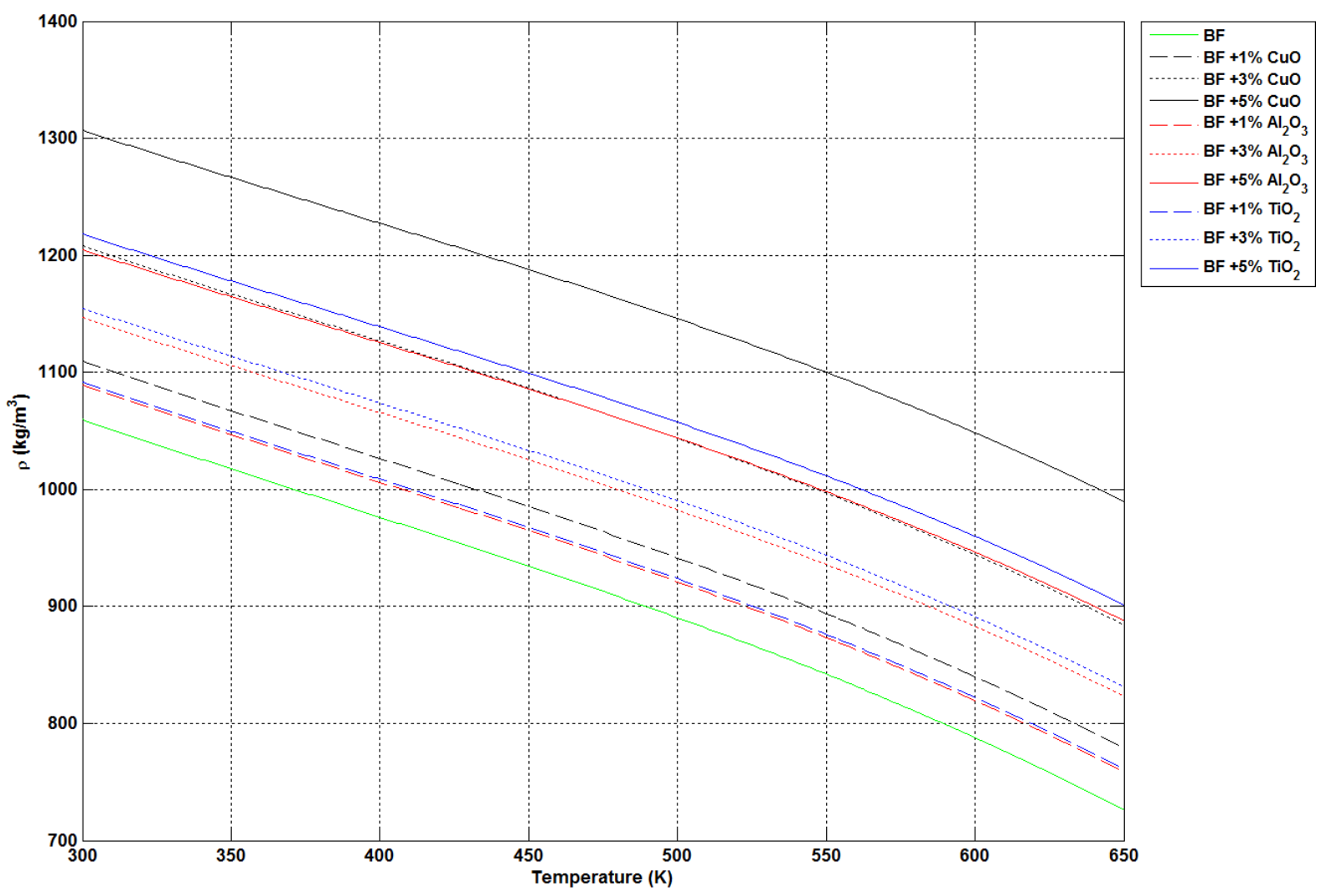




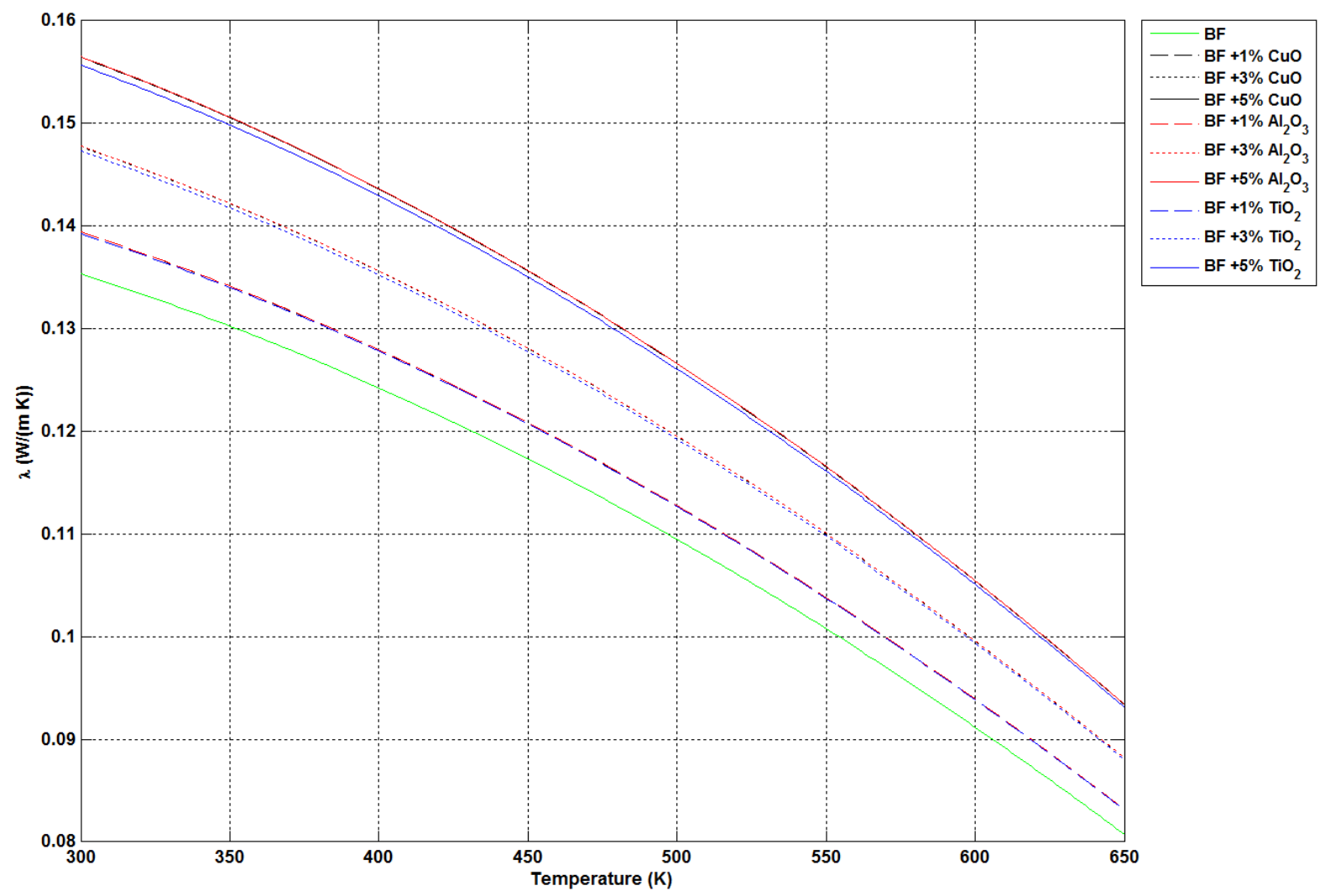


631

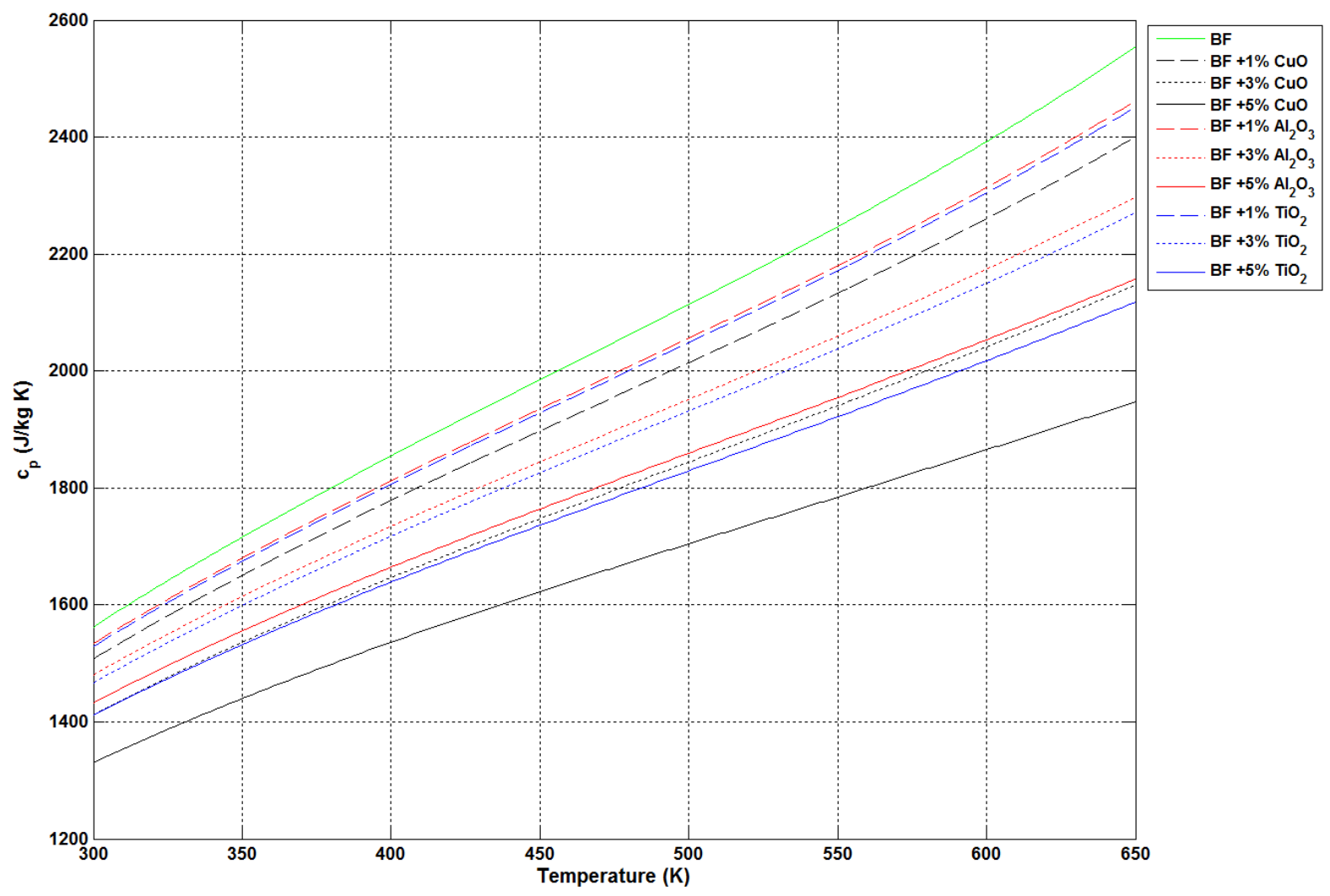

633

(c) Specific heat capacity 


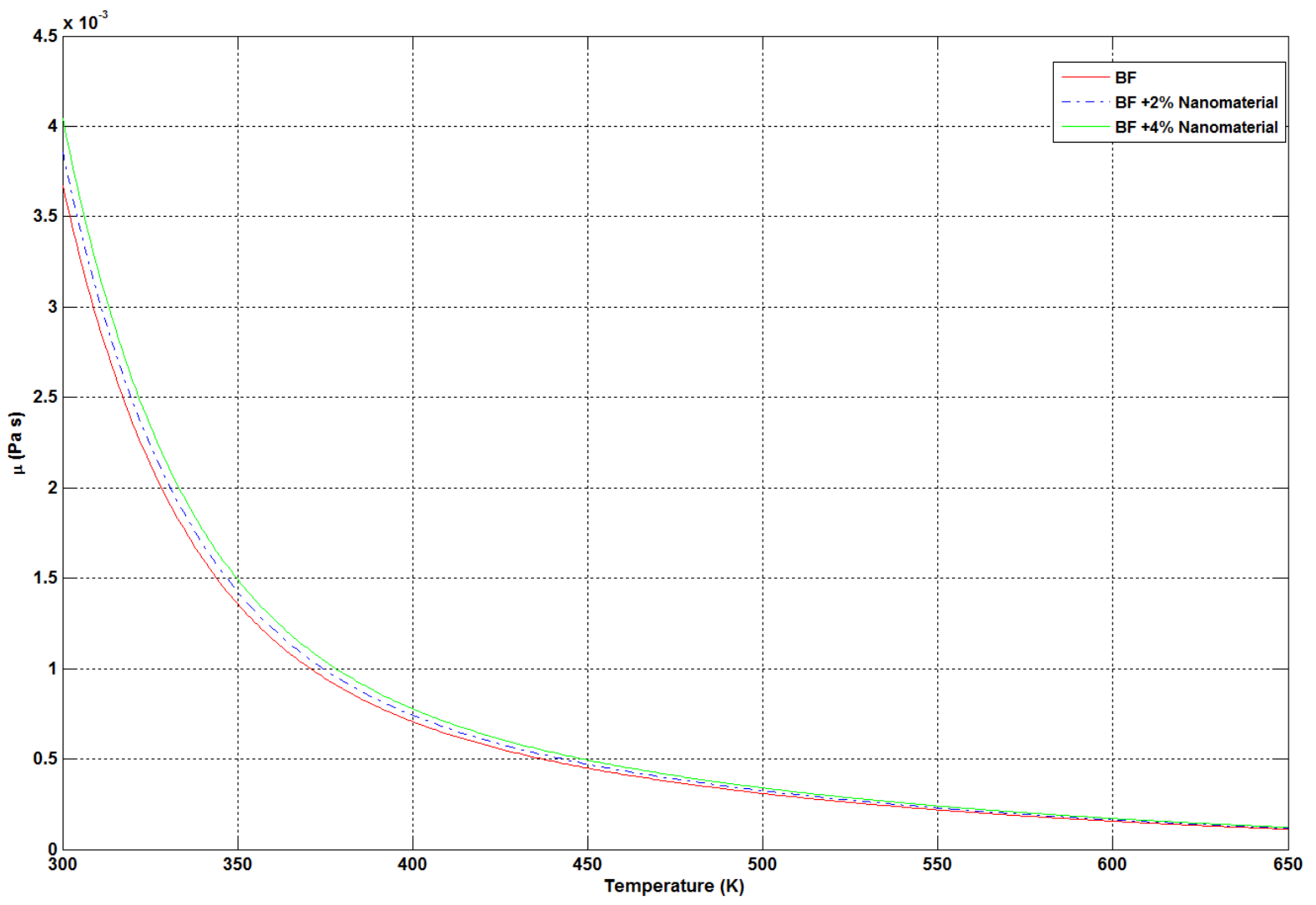

637

(d) Dynamic viscosity

638

639

640

Fig. 4: Thermal properties of base fluid and nanofluids

641 


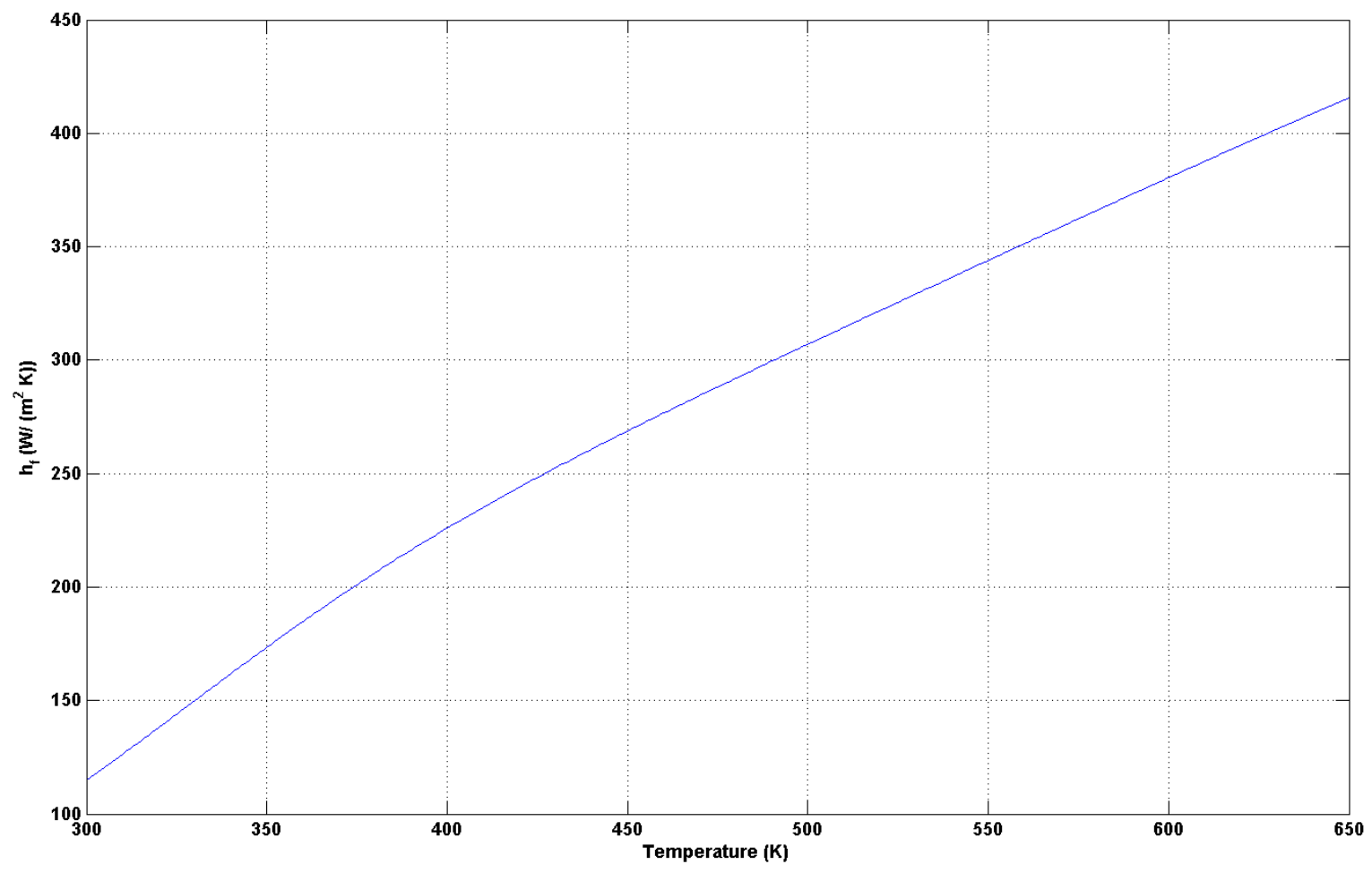

642

643 Fig. 5: Convective heat transfer coefficient for various fluid temperatures (base fluid)

644

645

646

647

648 


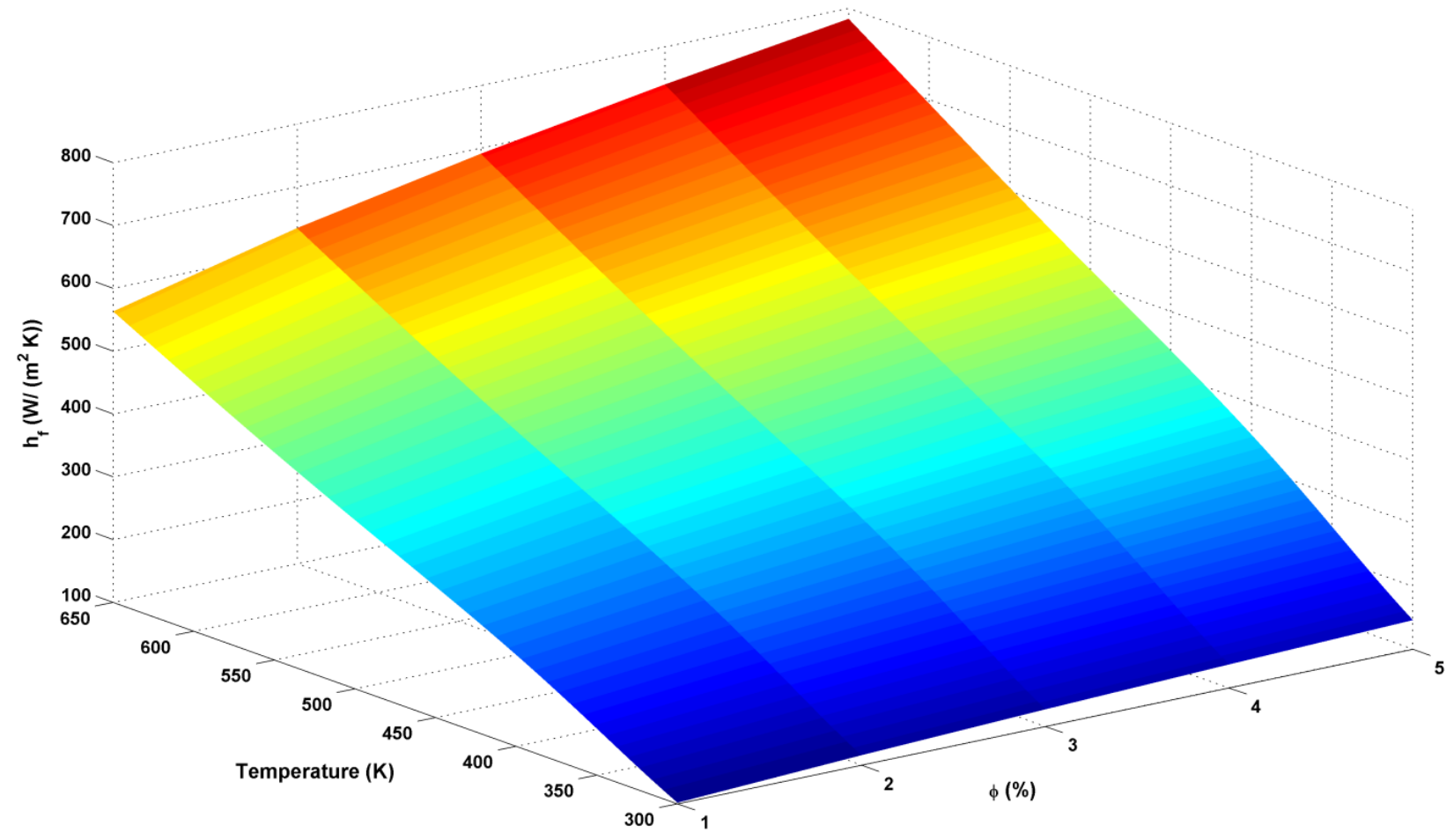

649

Fig. 6: Convective heat transfer coefficient for various fluid temperatures and 651 nanoparticle concentrations ( $\mathrm{CuO}$ based nanofluid)

652 


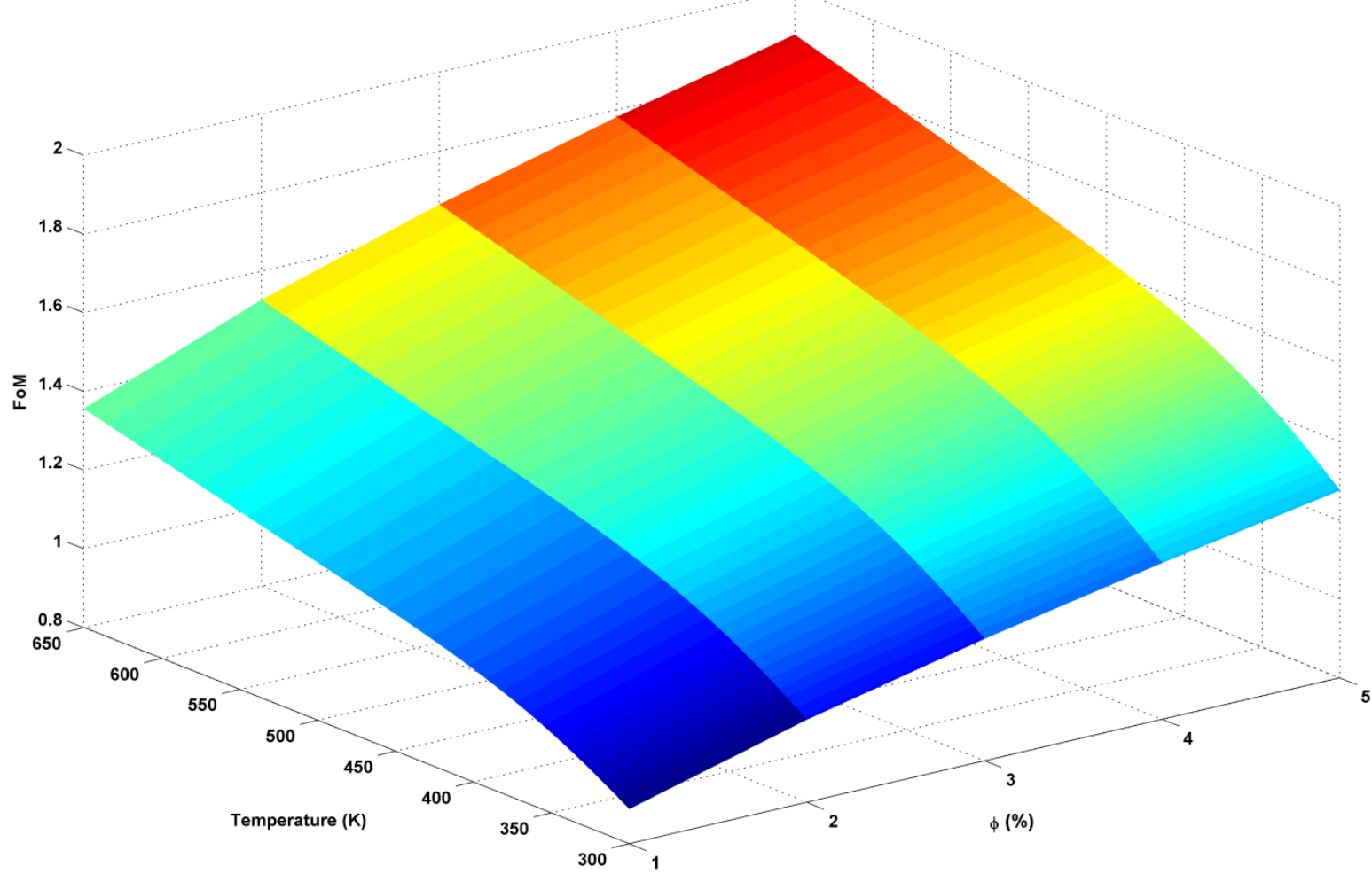

Fig. 7: Figure of Merit of Cuo based nanofluid for various fluid temperatures and 655 nanoparticle concentrations 


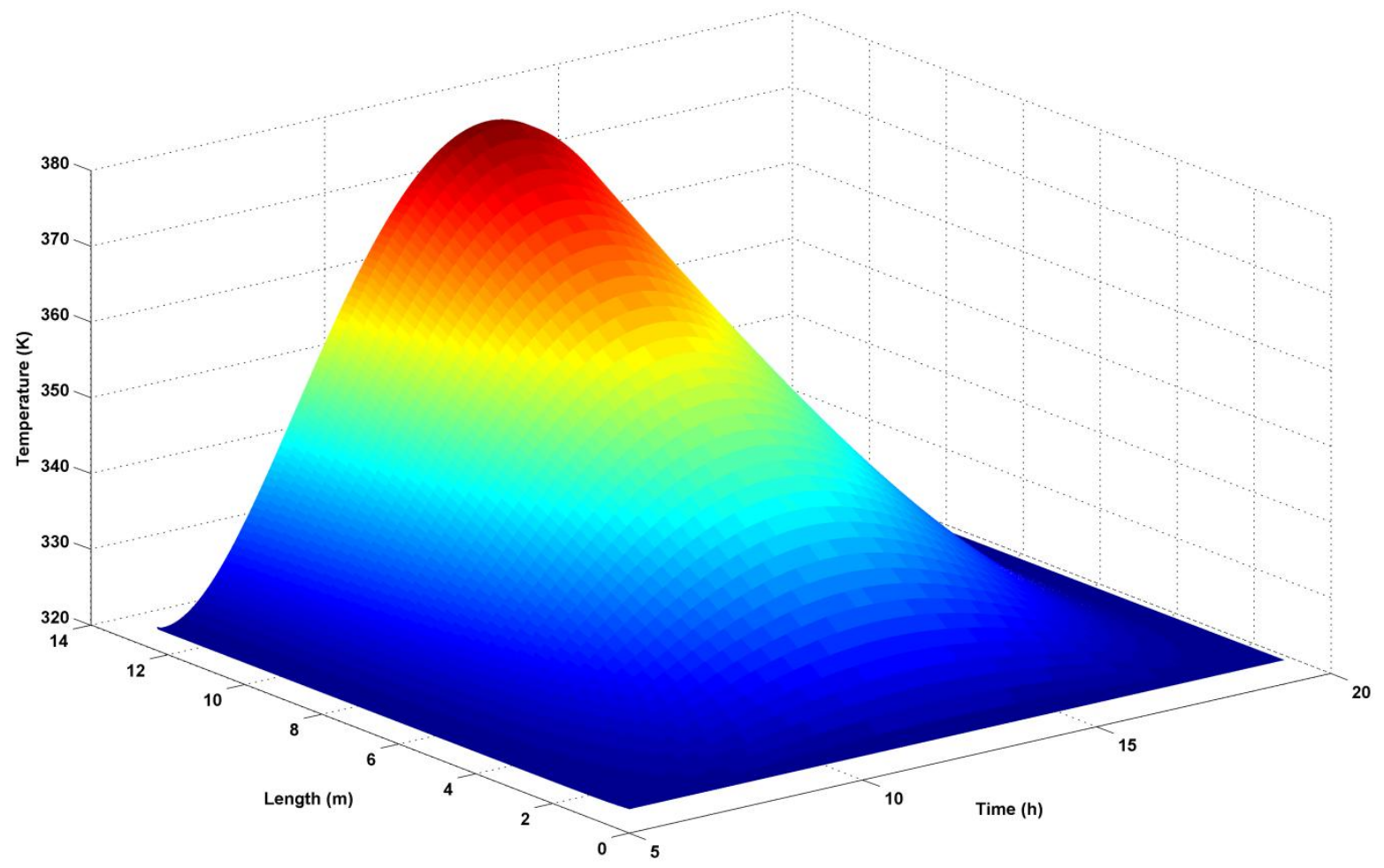

660 Fig. 8: Evolution of the base fluid temperature along the axial direction versus the time 


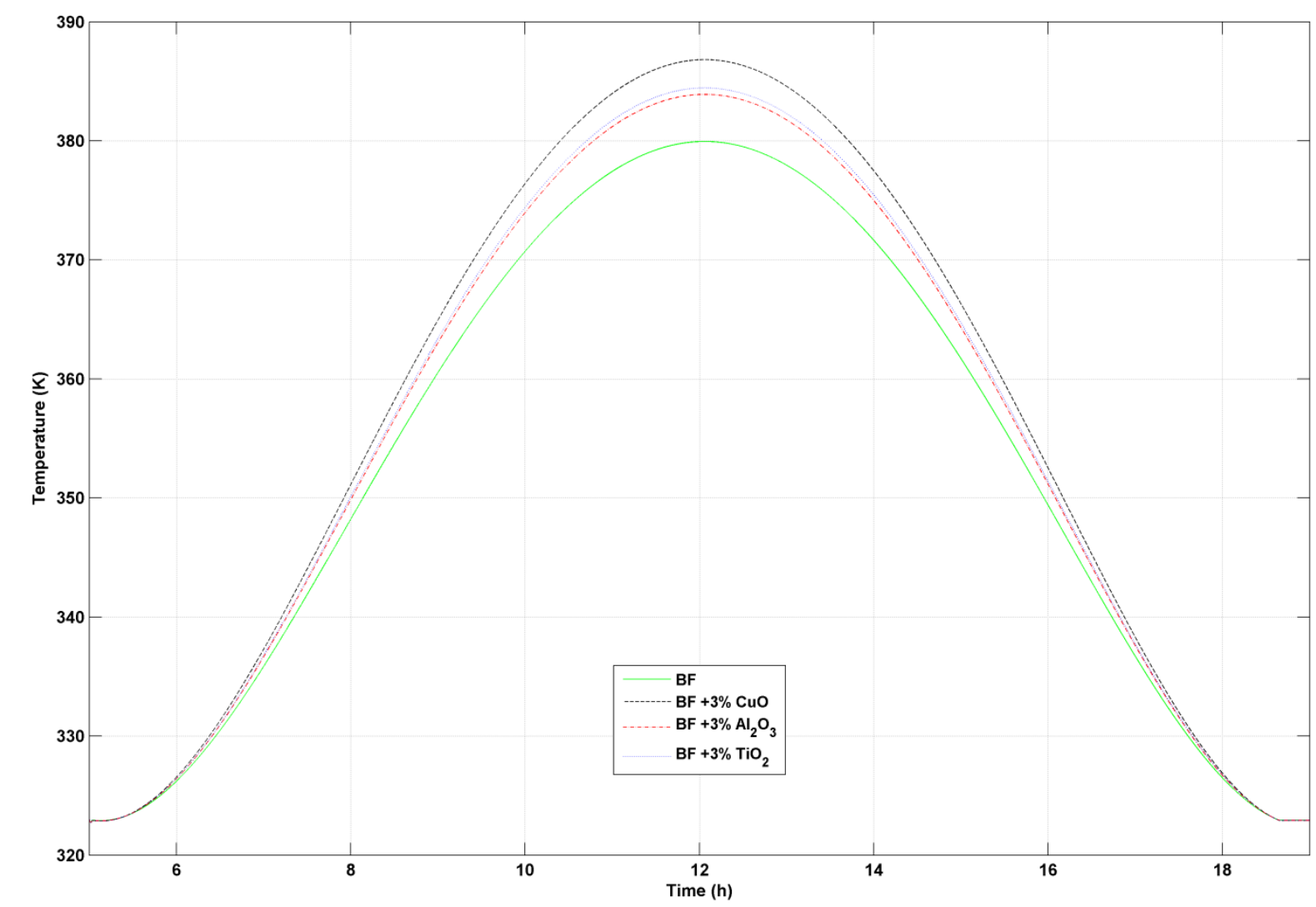

662

663 Fig. 9: Temporary evolution of outlet temperature of PTC (comparison between base 664 fluid and nanofluids) 
666

667 668

669

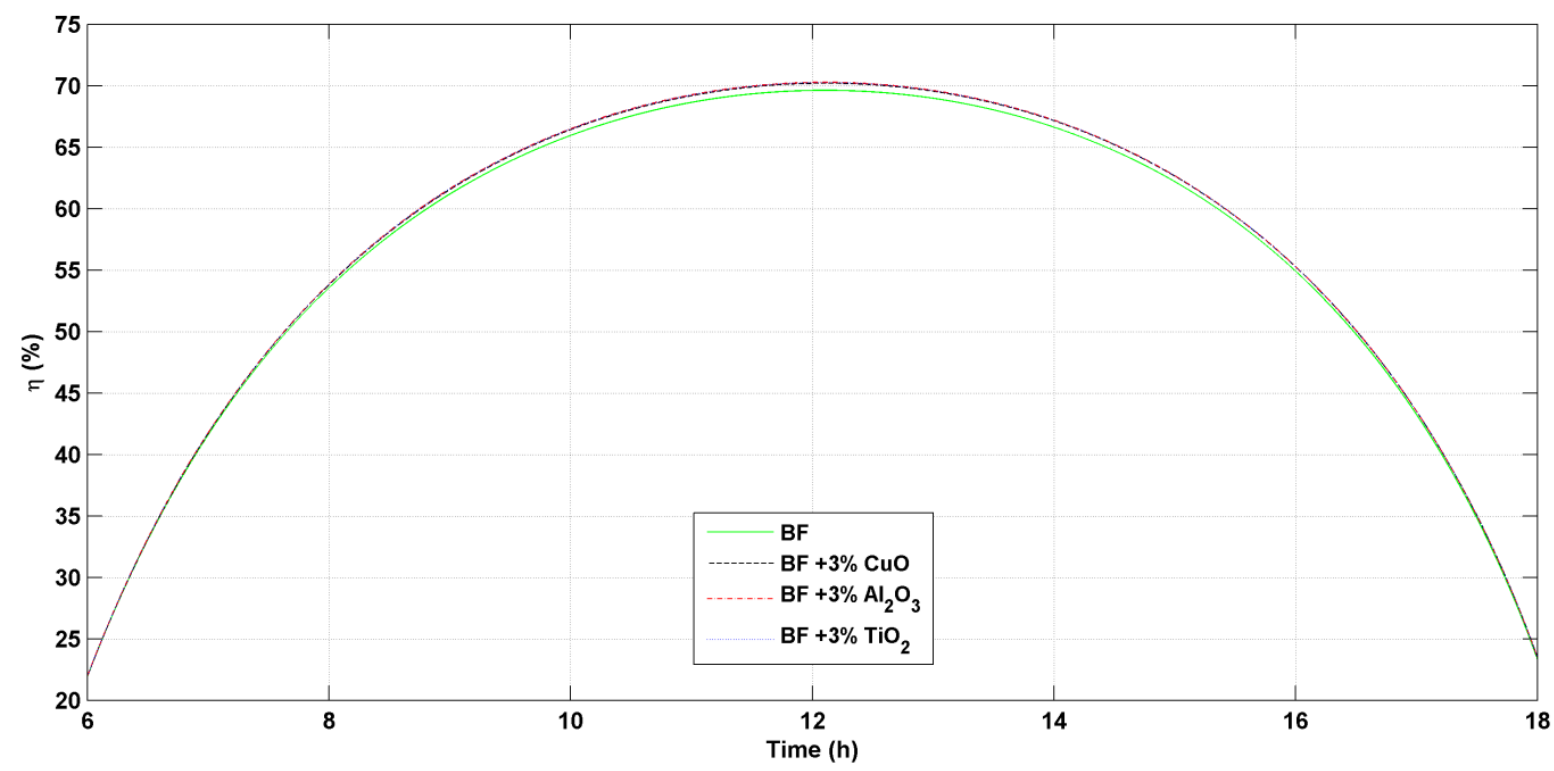

Fig. 10: Temporary evolution of thermal efficiency (comparison between base fluid and nanofluids) 
670

671

672

673

674

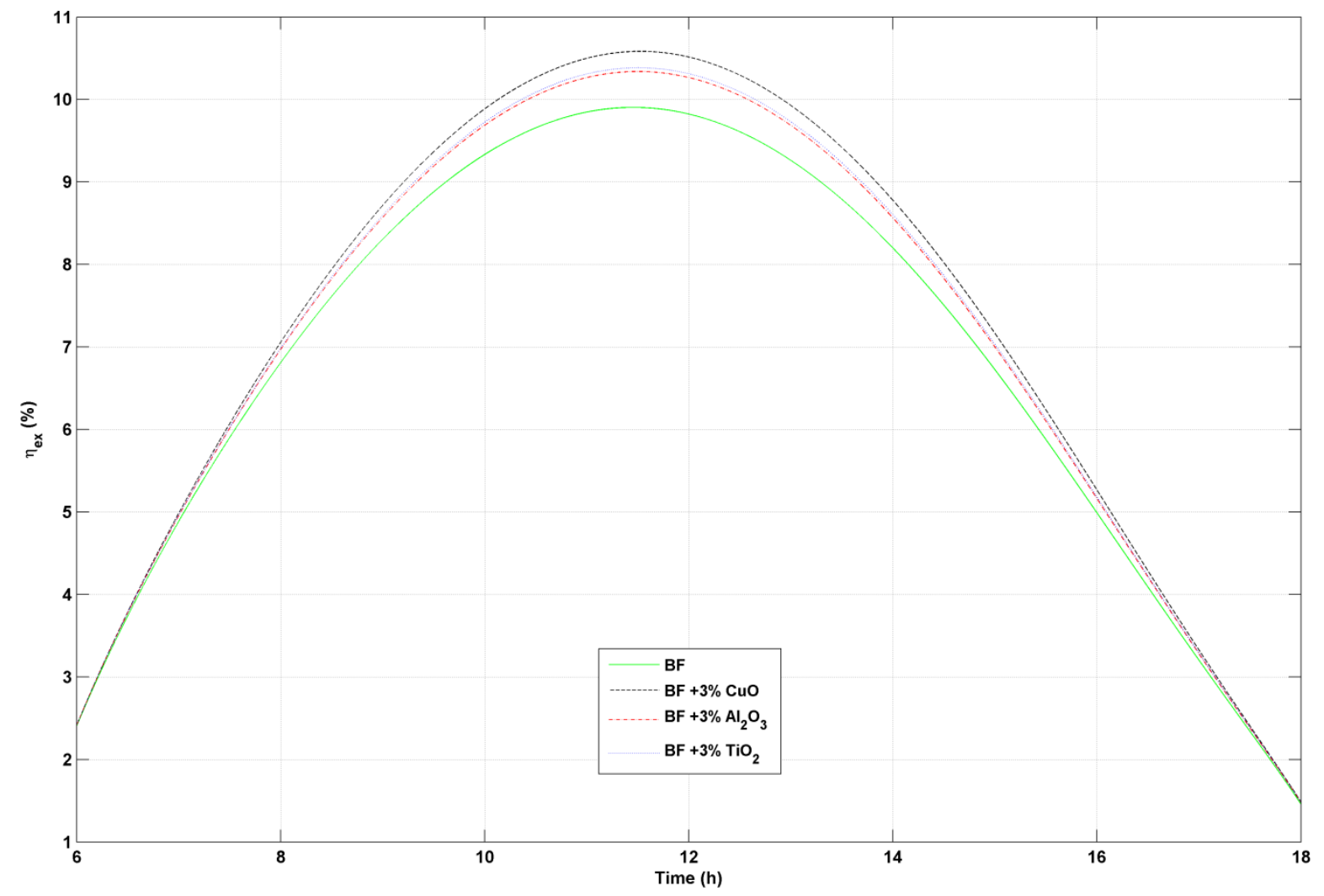

Fig. 11: Temporary evolution of exergy efficiency (comparison between base fluid and nanofluids) 


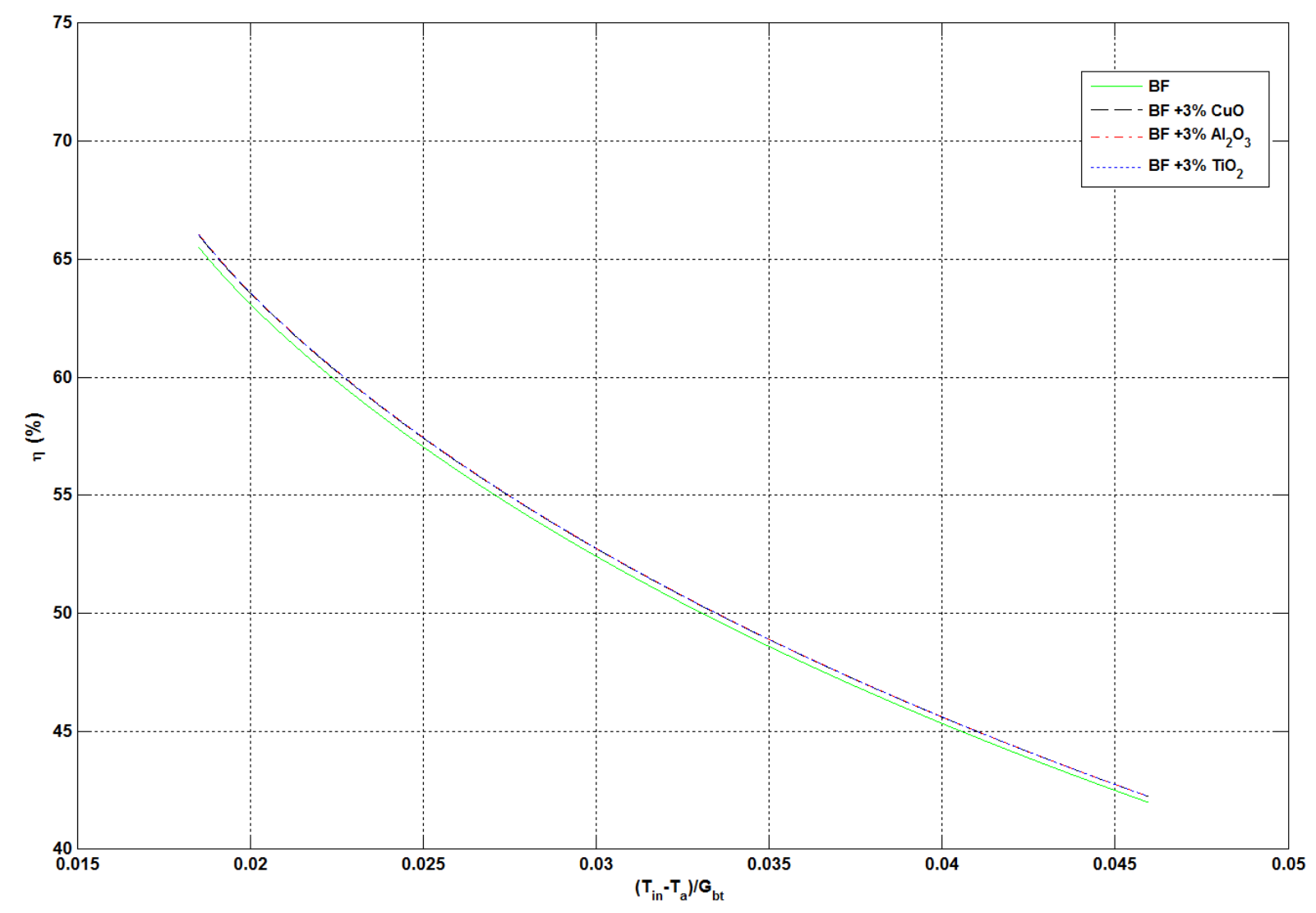

676

(a) Thermal efficiency

678 
680

681

682

683

684

685

686

687

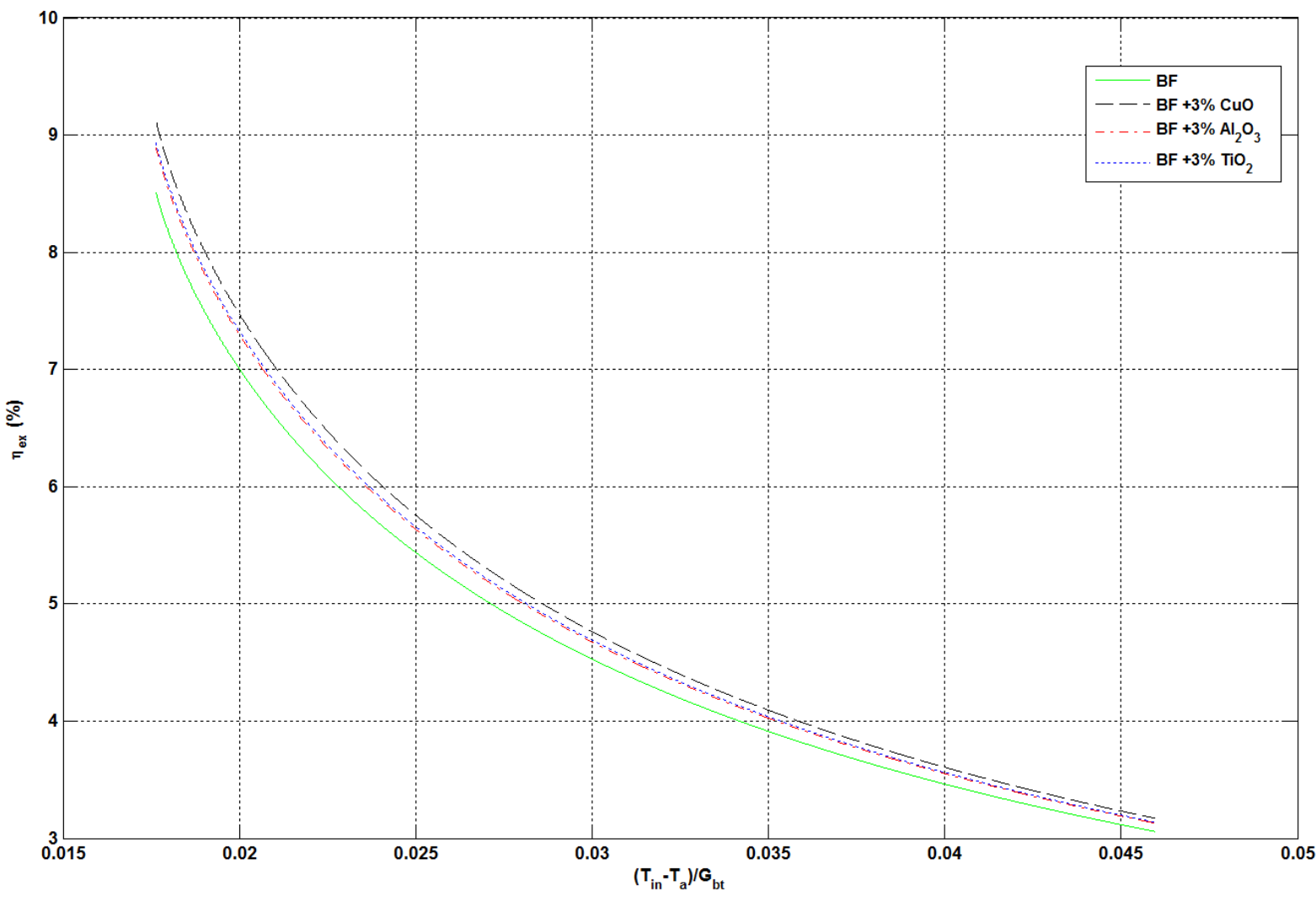

(b) Exergy efficiency

Fig. 12 : Comparison of thermal/exergy efficiency (base fluid and nanofluids) 


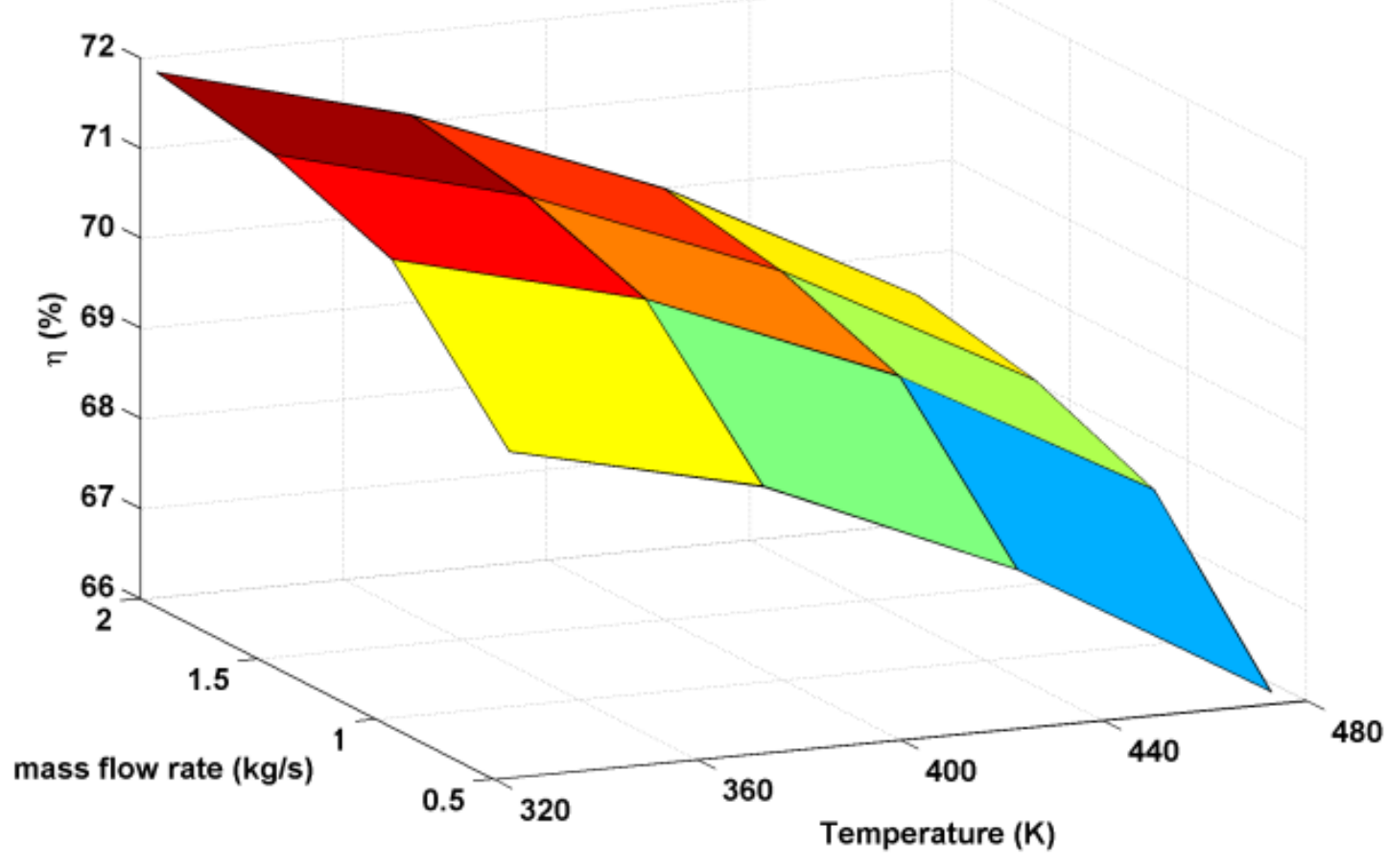

688

689

(a) Base fluid

690

691

692

693

694

695

696

697 


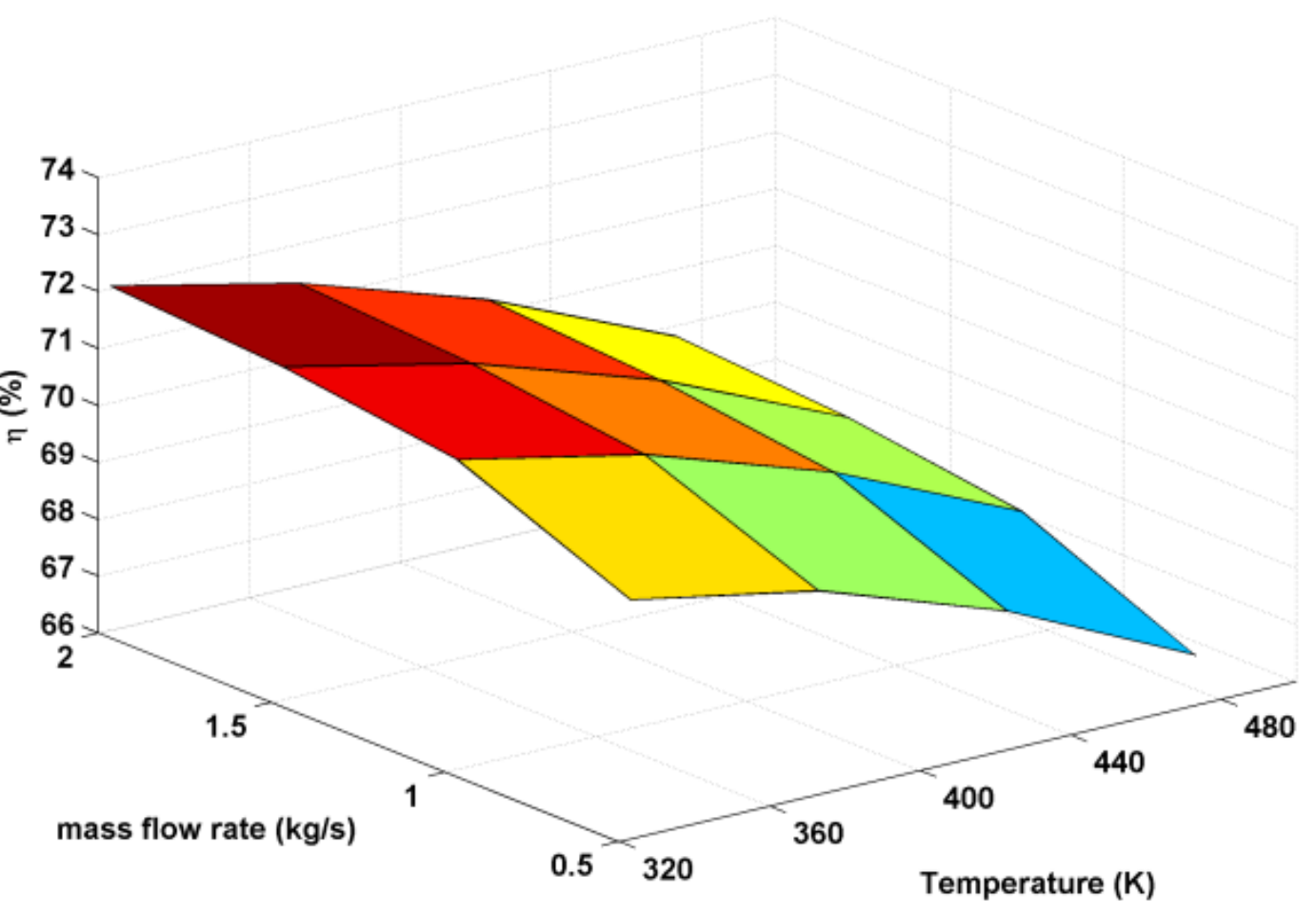

699

700

701

(b) CuO based nanofluid ( $\phi=3 \%$ )

702

703

704

705

706

Fig. 13: Thermal efficiency for various inlet temperatures and mass flow rates 


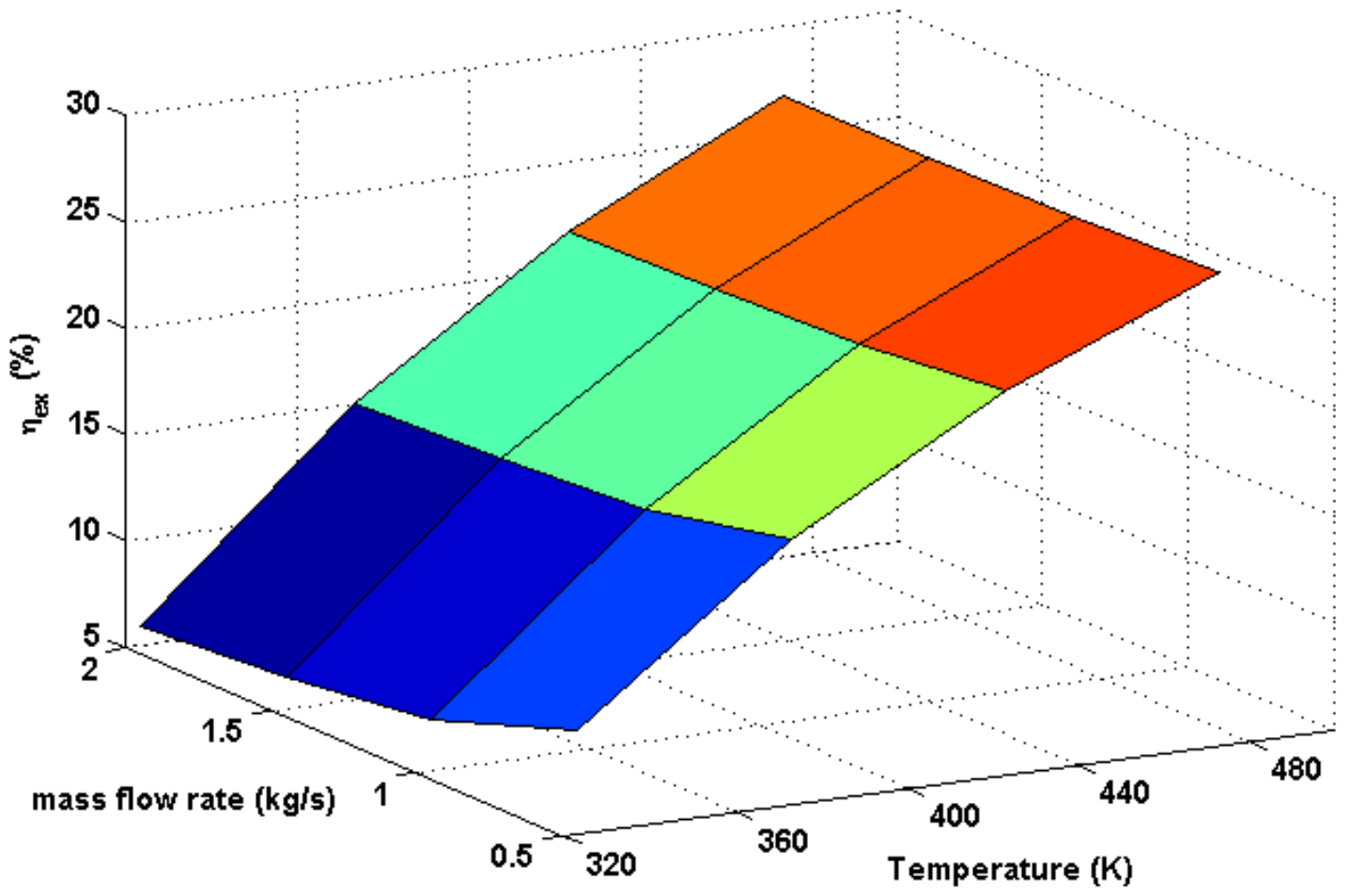

708

709

710

(a) Base fluid

711

712

713

714

715

716

717 


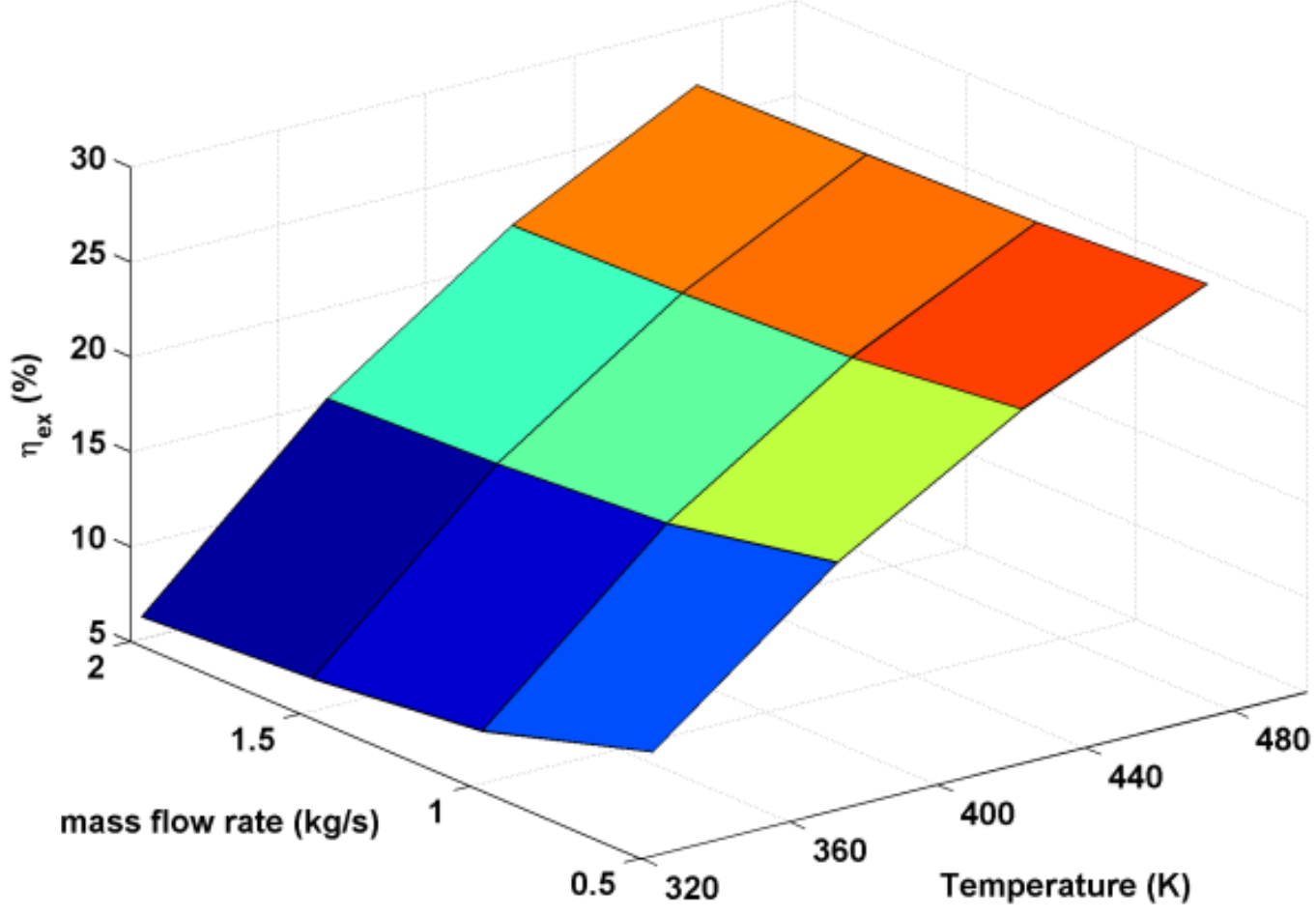

(b) CuO based nanofluid ( $\phi=3 \%)$

725 
Table 1: Properties of the used nanomaterials

\begin{tabular}{|l|l|l|l|}
\hline Material & $\begin{array}{l}\text { Specific heat } \\
(\mathbf{J} / \mathbf{k g ~ K})\end{array}$ & $\begin{array}{l}\text { Thermal conductivity } \\
(\mathbf{W} / \mathbf{m} \text { K) }\end{array}$ & Density $\left(\mathbf{k g} / \mathbf{m}^{\mathbf{3}}\right)$ \\
\hline Copper Oxide $(\mathrm{CuO})$ & 551 & 33 & 6000 \\
\hline Alumina $\left(\mathrm{Al}_{2} \mathrm{O}_{3}\right)$ & 773 & 40 & 3960 \\
\hline Titanium Oxide $\left(\mathrm{TiO}_{2}\right)$ & 692 & 8.4 & 4230 \\
\hline
\end{tabular}


Table 2: Geometrical and optical properties of PTC [37]

\begin{tabular}{|l|l|}
\hline Parameter & Value \\
\hline Length of the collector $(\mathrm{L})$ & $12.27 \mathrm{~m}$ \\
\hline Width of the collector & $5.76 \mathrm{~m}$ \\
\hline Receiver inner diameter & $0.066 \mathrm{~m}$ \\
\hline Receiver outer diameter & $0.07 \mathrm{~m}$ \\
\hline Glass envelope inner diameter & $0.115 \mathrm{~m}$ \\
\hline Glass envelope outer diameter & $0.121 \mathrm{~m}$ \\
\hline Absorptance of the receiver $(\alpha)$ & 0.96 \\
\hline Transmittance of the glass cover $(\tau)$ & 0.96 \\
\hline Reflectance of the mirror $\left(\mathrm{r}_{\mathrm{m}}\right)$ & 0.94 \\
\hline Intercept factor $(\gamma)$ & 0.867 \\
\hline
\end{tabular}

736 
Table 3: Comparison of model prediction with experimental tests from SNL [44]

\begin{tabular}{|c|c|c|c|c|c|c|c|c|c|c|c|}
\hline \multirow{2}{*}{ Test } & \multicolumn{5}{|c|}{ Test conditions } & \multicolumn{3}{|c|}{ Outlet Temperature (K) } & \multicolumn{3}{|c|}{ Thermal Efficiency (\%) } \\
\hline & $\mathrm{DNI}\left(\mathrm{W} / \mathrm{m}^{2}\right)$ & Wind $(\mathrm{m} / \mathrm{s})$ & $\mathrm{T}_{\mathrm{amb}}(\mathrm{K})$ & $\mathrm{T}_{\text {in }}(\mathrm{K})$ & \begin{tabular}{|l|}
$\begin{array}{l}\text { mass flow rate } \\
(\mathrm{kg} / \mathrm{s})\end{array}$ \\
\end{tabular} & SNL test & model & Deviation $(\mathrm{K})$ & SNL test & model & Deviation $(\%)$ \\
\hline State 1 & 933.7 & 2.60 & 294.35 & 375.35 & 0.66 & 397.15 & 397.08 & 0.07 & 72.51 & 69.61 & 2.9 \\
\hline State 2 & 968.2 & 3.70 & 295.55 & 424.15 & 0.68 & 446.45 & 446.07 & 0.38 & 70.9 & 69.84 & 1.06 \\
\hline State 3 & 937.9 & 1.00 & 301.95 & 570.95 & 0.61 & 590.05 & 590.88 & 0.83 & 67.98 & 66.64 & 1.34 \\
\hline
\end{tabular}

740 
Table 4: Relative energy gains using nanofluids instead of base fluid (effect of mass flow

\begin{tabular}{|c|c|c|c|c|c|c|c|c|}
\hline \multirow[b]{3}{*}{ HTF } & \multicolumn{8}{|c|}{ Mass flow rate $(\mathrm{kg} / \mathrm{s})$} \\
\hline & \multicolumn{2}{|c|}{0.5} & \multicolumn{2}{|l|}{1} & \multicolumn{2}{|c|}{1.5} & \multicolumn{2}{|l|}{2} \\
\hline & $\mathbf{Q}(\mathbf{k W h})$ & $\Delta \mathrm{e}(\%)$ & $\mathbf{Q}(\mathbf{k W h})$ & $\Delta \mathrm{e}(\%)$ & $\mathbf{Q}(\mathbf{k W h})$ & $\Delta \mathrm{e}(\%)$ & $\mathbf{Q}(\mathbf{k W h})$ & $\Delta \mathrm{e}(\%)$ \\
\hline $\mathrm{BF}$ & 358.4 & & 365.3 & & 367.7 & & 369 & \\
\hline $\mathrm{BF}+1 \% \mathrm{CuO}$ & 358.2 & -0.0558 & 365.7 & 0.1095 & 368.2 & 0.136 & 369.4 & 0.1084 \\
\hline $\mathrm{BF}+2 \% \mathrm{CuO}$ & 359.9 & 0.4185 & 366.5 & 0.3285 & 368.7 & 0.272 & 369.8 & 0.2168 \\
\hline $\mathrm{BF}+3 \% \mathrm{CuO}$ & 360.9 & 0.6975 & 367 & 0.4654 & 369 & 0.3535 & 370 & 0.271 \\
\hline $\mathrm{BF}+4 \% \mathrm{CuO}$ & 361.7 & 0.9208 & 367.4 & 0.5749 & 369.3 & 0.4351 & 370.2 & 0.3252 \\
\hline $\mathrm{BF}+5 \% \mathrm{CuO}$ & 362.2 & 1.0603 & 367.6 & 0.6296 & 369.4 & 0.4623 & 370.3 & 0.3523 \\
\hline $\mathrm{BF}+1 \% \mathrm{Al}_{2} \mathrm{O}_{3}$ & 358.4 & -0.0088 & 365.8 & 0.1369 & 368.2 & 0.136 & 369.5 & 0.1355 \\
\hline $\mathrm{BF}+2 \% \mathrm{Al}_{2} \mathrm{O}_{3}$ & 360.1 & 0.4743 & 366.6 & 0.3559 & 368.8 & 0.2992 & 369.9 & 0.2439 \\
\hline $\mathrm{BF}+3 \% \mathrm{Al}_{2} \mathrm{O}_{3}$ & 361.3 & 0.8092 & 367.2 & 0.5201 & 369.1 & 0.3807 & 370.1 & 0.2981 \\
\hline $\mathrm{BF}+4 \% \mathrm{Al}_{2} \mathrm{O}_{3}$ & 362 & 1.0045 & 367.5 & 0.6022 & 369.4 & 0.4623 & 370.3 & 0.3523 \\
\hline $\mathrm{BF}+5 \% \mathrm{Al}_{2} \mathrm{O}_{3}$ & 362.6 & 1.1719 & 367.8 & 0.6844 & 369.6 & 0.5167 & 370.5 & 0.4065 \\
\hline $\mathrm{BF}+1 \% \mathrm{TiO}_{2}$ & 358.3 & -0.0279 & 365.8 & 0.1369 & 368.2 & 0.136 & 369.4 & 0.1084 \\
\hline $\mathrm{BF}+2 \% \mathrm{TiO}_{2}$ & 360.1 & 0.4743 & 366.6 & 0.3559 & 368.8 & 0.2992 & 369.8 & 0.2168 \\
\hline $\mathrm{BF}+3 \% \mathrm{TiO}_{2}$ & 361.2 & 0.7813 & 367.1 & 0.4927 & 369.1 & 0.3807 & 370.1 & 0.2981 \\
\hline $\mathrm{BF}+4 \% \mathrm{TiO}_{2}$ & 361.9 & 0.9766 & 367.5 & 0.6022 & 369.4 & 0.4623 & 370.3 & 0.3523 \\
\hline $\mathrm{BF}+5 \% \mathrm{TiO}_{2}$ & 362.5 & 1.144 & 367.8 & 0.6844 & 369.5 & 0.4895 & 370.4 & 0.3794 \\
\hline
\end{tabular}




\begin{tabular}{|c|c|c|c|c|c|c|c|c|}
\hline \multirow{3}{*}{ HTF } & \multicolumn{8}{|c|}{ Inlet Temperature (K) } \\
\hline & \multicolumn{2}{|l|}{323} & \multicolumn{2}{|l|}{373} & \multicolumn{2}{|l|}{423} & \multicolumn{2}{|l|}{473} \\
\hline & $\mathbf{Q}(\mathbf{k W h})$ & $\Delta \mathrm{e}(\%)$ & $\mathbf{Q}(\mathbf{k W h})$ & $\Delta \mathrm{e}(\%)$ & $\mathbf{Q}(\mathbf{k W h})$ & $\Delta \mathrm{e}(\%)$ & $\mathbf{Q}(\mathbf{k W h})$ & $\Delta \mathrm{e}(\%)$ \\
\hline$\overline{\mathrm{BF}}$ & 358.4 & & 351.9 & & 341.9 & & 328.9 & \\
\hline $\mathrm{BF}+1 \% \mathrm{CuO}$ & 358.2 & -0.0558 & 352.7 & 0.2273 & 343.3 & 0.4095 & 330.8 & 0.5777 \\
\hline $\mathrm{BF}+2 \% \mathrm{CuO}$ & 359.9 & 0.4185 & 353.9 & 0.5683 & 344.4 & 0.7312 & 331.8 & 0.8817 \\
\hline $\mathrm{BF}+3 \% \mathrm{CuO}$ & 360.9 & 0.6975 & 354.7 & 0.7957 & 345 & 0.9067 & 332.4 & 1.0642 \\
\hline $\mathrm{BF}+4 \% \mathrm{CuO}$ & 361.7 & 0.9208 & 355.2 & 0.9378 & 345.4 & 1.0237 & 332.8 & 1.1858 \\
\hline $\mathrm{BF}+5 \% \mathrm{CuO}$ & 362.2 & 1.0603 & 355.5 & 1.023 & 345.7 & 1.1114 & 333 & 1.2466 \\
\hline $\mathrm{BF}+1 \% \mathrm{Al}_{2} \mathrm{O}_{3}$ & 358.4 & $\begin{array}{c}-0.0088 \\
\end{array}$ & 352.8 & 0.2558 & 343.4 & 0.4387 & 330.9 & 0.6081 \\
\hline $\mathrm{BF}+2 \% \mathrm{Al}_{2} \mathrm{O}_{3}$ & 360.1 & 0.4743 & 354.1 & 0.6252 & 344.6 & 0.7897 & 332.1 & 0.9729 \\
\hline $\mathrm{BF}+3 \% \mathrm{Al}_{2} \mathrm{O}_{3}$ & 361.3 & 0.8092 & 355 & 0.8809 & 345.4 & 1.0237 & 332.8 & 1.1858 \\
\hline $\mathrm{BF}+4 \% \mathrm{Al}_{2} \mathrm{O}_{3}$ & 362 & 1.0045 & 355.6 & 1.0514 & 345.9 & 1.1699 & 333.3 & 1.3378 \\
\hline $\mathrm{BF}+5 \% \mathrm{Al}_{2} \mathrm{O}_{3}$ & 362.6 & 1.1719 & 356 & 1.1651 & 346.3 & 1.2869 & 333.7 & 1.4594 \\
\hline $\mathrm{BF}+1 \% \mathrm{TiO}_{2}$ & 358.3 & -0.0279 & 352.8 & 0.2558 & 343.4 & 0.4387 & 330.9 & 0.6081 \\
\hline $\mathrm{BF}+2 \% \mathrm{TiO}_{2}$ & 360.1 & 0.4743 & 354.1 & 0.6252 & 344.6 & 0.7897 & 332 & 0.9425 \\
\hline $\mathrm{BF}+3 \% \mathrm{TiO}_{2}$ & 361.2 & 0.7813 & 354.9 & 0.8525 & 345.3 & 0.9944 & 332.7 & 1.1554 \\
\hline $\mathrm{BF}+4 \% \mathrm{TiO}_{2}$ & 361.9 & 0.9766 & 355.5 & 1.023 & 345.8 & 1.1407 & 333.2 & 1.3074 \\
\hline $\mathrm{BF}+5 \% \mathrm{TiO}_{2}$ & 362.5 & 1.144 & 355.9 & 1.1367 & 346.2 & 1.2577 & 333.5 & 1.3986 \\
\hline
\end{tabular}

